Linköping Studies in Science and Technology

Dissertation No. 1810

\title{
Tunable and modular assembly of polypeptides and polypeptide-hybrid biomaterials
}

\author{
Christopher Aronsson
}

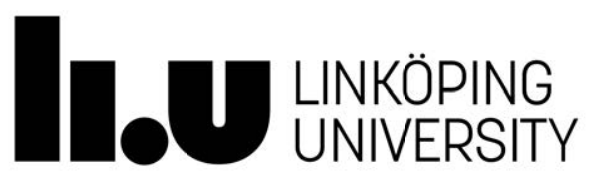

Division of Molecular Physics

Department of Physics, Chemistry and Biology

Linköping University, Sweden

Linköping 2016 
During the course of the research underlying this thesis, Christopher Aronsson was enrolled in Forum Scientium, a multidisciplinary doctoral programme at Linköping University, Sweden.

๑ Copyright 2016 Christopher Aronsson, unless otherwise noted

Aronsson, Christopher

Tunable and modular assembly of polypeptides and polypeptide-hybrid biomaterials

ISBN: 978-91-7685-627-7

ISSN: 0345-7524

Linköping Studies in Science and Technology, Dissertation No. 1810

Electronic publication: http://www.ep.liu.se

Printed in Sweden by LiU-Tryck, Linköping 2016 
"Science, my boy, is made up of mistakes, but they are mistakes which it is useful to make, because they lead little by little to the truth."

Fules Verne, fourney to the Center of the Earth (1864) 



\section{Abstract}

Biomaterials are materials that are specifically designed to be in contact with biological systems and have for a long time been used in medicine. Examples of biomaterials range from sophisticated prostheses used for replacing outworn body parts to ordinary contact lenses. Currently it is possible to create biomaterials that can e.g. specifically interact with cells or respond to certain stimuli. Peptides, the shorter version of proteins, are excellent molecules for fabrication of such biomaterials. By following and developing design rules it is possible to obtain peptides that can self-assemble into well-defined nanostructures and biomaterials.

The aim of this thesis is to create "smart" and tunable biomaterials by molecular self-assembly using dimerizing $\alpha$-helical polypeptides. Two different, but structurally related, polypeptide-systems have been used in this thesis. The EKIV-polypeptide system was developed in this thesis and consists of four 28-residue polypeptides that can be mixed-and-matched to self-assemble into four different coiled coil heterodimers. The dissociation constant of the different heterodimers range from $\mu \mathrm{M}$ to $<\mathrm{nM}$. Due to the large difference in affinities, the polypeptides are prone to thermodynamic social self-sorting. The JR-polypeptide system, on the other hand, consists of several 42-residue de novo designed helix-loop-helix polypeptides that can dimerize into four-helix bundles. In this work, primarily the glutamic acid-rich polypeptide JR2E has been explored as a component in supramolecular materials. Dimerization was induced by exposing the polypeptide to either $\mathrm{Zn}^{2+}$, acidic conditions or the complementary polypeptide JR2K.

By conjugating JR2E to hyaluronic acid and the EKIV-polypeptides to star-shaped poly(ethylene glycol), respectively, highly tunable hydrogels that can be self-assembled in a modular fashion have been created. In addition, self-assembly of spherical superstructures has been investigated and were obtained by linking two thiol-modified JR2E polypeptides via a disulfide bridge in the loop region. The thesis also demonstrates that the polypeptides and the polypeptide-hybrids can be used for encapsulation and release of molecules and nanoparticles. In addition, some of the hydrogels have been explored for 3D cell culture. By using supramolecular interactions combined with bio-orthogonal 
covalent crosslinking reactions, hydrogels were obtained that enabled facile encapsulation of cells that retained high viability.

The results of the work presented in this thesis show that dimerizing $\alpha$-helical polypeptides can be used to create modular biomaterials with properties that can be tuned by specific molecular interactions. The modularity and the tunable properties of these smart biomaterials are conceptually very interesting and make them useful in many emerging biomedical applications, such as 3D cell culture, cell therapy, and drug delivery. 


\section{Populärvetenskaplig sammanfattning}

Biomaterial är material som är designade för att vara i kontakt med biologiskt material och har använts länge inom medicinen. Exempel på biomaterial inkluderar allt från avancerade proteser som används för att ersätta kroppsdelar till helt vanliga kontaktlinser. Genom smart design kan man idag skapa biomaterial som kan interagera med celler på ett förutsägbart sätt och vars mekaniska och strukturella egenskaper kan styras med molekylär precision. Peptider är kortare varianter av proteiner och är ett exempel på molekyler som kan användas för att på molekylär nivå kontrollera ett biomaterials egenskaper. Med den kunskap som finns idag kan vi designa och tillverka peptider som har ytterst specifika funktioner. Peptider kan bland annat användas för att förbättra cellers tillväxt, döda skadliga bakterier, styra de mekaniska egenskaperna hos ett biomaterial eller användas för att skapa biomaterial som kan reagera och förändra sina egenskaper när faktorer såsom temperatur, $\mathrm{pH}$ eller jon-halt förändras.

Den här avhandlingen handlar om hur man via ett Lego-liknande tillvägagångssätt kan tillverka "smarta" biomaterial där peptider används både som byggklossar och för att förmå byggklossarna att spontant bilda större strukturer och material. Peptiderna som har använts bildar nanometer-små (miljondels millimeter) spiraler som kallas alfa-helixar, vilka i sin tur aggregerar parvis för att skapa stabila strukturer. Bildandet av dessa stabila strukturer kan man i sin tur styra på flera sätt, tex genom att ändra $\mathrm{pH}$ eller förekomst av specifika joner som zink-joner. Genom att sätta fast peptiderna på den naturligt förekommande polymeren hyaluronsyra samt den syntetiska polymeren polyetylenglykol kan peptidernas förmåga att bilda alfa-helixar användas för att skapa modulära och justerbara hydrogeler. Hydrogeler består av mer än $90 \%$ vatten vilket gör dem ytterst lämpliga som biomaterial. Avhandlingen visar även hur dessa peptider och hydrogeler kan användas för att kapsla in och frisätta molekyler och nanopartiklar. Några av hydrogelerna har även använts för att odla celler i så kallad tre-dimensionell cellodling. Genom att använda cell-vänlig kemi kan biomaterialen skapas i närvaro av celler utan att cellerna tar skada och erbjuder förhållanden som efterliknar cellernas naturliga miljö. 
Resultatet av arbetet som presenteras i avhandlingen visar att den typ av peptider som har använts ger möjlighet att skapa fullt modulära biomaterial vars egenskaper kan justeras och påverkas på molekylär nivå. De modulära och justerbara egenskaper gör dessa smarta biomaterial intressanta för flertalet medicinska tillämpningar, såsom tre-dimensionell cellodling, regenerativ medicin samt för kontrollerad frisättning av läkemedel. 


\section{List of Publications}

This thesis is based on the following publications:

\section{Paper I}

C. Aronsson, S. Dånmark, F. Zhou, P. Öberg, K. Enander, H. Su, D. Aili Self-sorting heterodimeric coiled coil peptides with defined and tuneable self-assembly properties

Scientific Reports 2015, 5:14063

Contribution: Designed the peptides and developed synthesizes and purification strategies. Planned, conducted and analyzed all experiments except for the molecular dynamic data. Wrote the main part of the manuscript.

\section{Paper II}

S. Dånmark ${ }^{\dagger}$, C. Aronsson ${ }^{\dagger}$, D. Aili

Tailoring supramolecular peptide-poly(ethylene glycol) hydrogels by coiled coil self-assembly and self-sorting

Biomacromolecules 2016, 17(6):2260-2267

Contribution: Synthesized the non-pegylated peptides. Participated in the planning of all experiments and in the analysis of the acquired data. Wrote a major part of the manuscript.

\section{Paper III}

C. Aronsson ${ }^{\dagger}$, R. Selegård ${ }^{\dagger}$, D. Aili

Zinc-triggered hierarchical self-assembly of fibrous helix-loop-helix peptide superstructures for controlled encapsulation and release

Macromolecules 2016, 49(18):6997-7003

Contribution: Planned, conducted and analyzed all experiments. Wrote the main part of the manuscript. 


\section{Paper IV}

R. Selegård, C. Aronsson, C. Brommesson, S. Dånmark, D. Aili Folding driven self-assembly of a stimuli-responsive peptide-hyaluronan hybrid hydrogel

Submitted 2016

Contribution: Planned, conducted and analyzed the rheological experiments. Participated in the planning and the analysis of the release experiments. Contributed to the final editing of the manuscript.

\section{Paper V}

C. Aronsson ${ }^{\dagger}$, R. Selegård ${ }^{\dagger}$, J. Christoffersson, C-F. Mandenius, D. Aili Supramolecular Functionalization and Tuning of Peptide Modified Bio-Orthogonally Crosslinked Hyaluronan-Poly(ethylene glycol) Hydrogels

Manuscript 2016

Contribution: Planned, conducted and analyzed all hydrogel characterization experiments. Planned, conducted and analyzed the cell studies with J.C. Wrote the main part of the manuscript.

This thesis contains unpublished data in addition to the included papers.

$\dagger$ These authors contributed equally to this work. 


\section{Publications not included in this thesis.}

M. Fürsatz, M. Skog, P. Sivlér, E. Palm, C. Aronsson, A. Skallberg, G. Greczynski, H. Khalaf, T. Bengtsson, D. Aili

Versatile methods for surface modification of fibrous bacterial cellulose membranes in aqueous conditions.

Manuscript 2016

\section{Conference contributions.}

C. Aronsson, S. Dånmark, F. Zhou, P. Öberg, H. Su, D. Aili Self-Sorting Heterodimeric Coiled Coil Peptides with Defined and Tunable Self-Assembly Properties. Nanopeptide 2015, 2015, Glasgow, UK.

S. Dånmark, C. Aronsson, D. Aili

Tailoring the properties of supramolecular nanomaterials by coiled coil polypeptide heterodimerization, Conference on Advanced Functional Materials, 2016, Kolmården, Sweden. 


\section{Abbreviations}

Alloc

AuNP

Allylcarboxycarbonyl

BCN

Gold nanoparticle

$\mathrm{CD}$

Bicyclo[6.1.0]nonyne

DLS

Circular dichroism

ECM

Dynamic light scattering

EDTA

Extracellular matrix

G'

Ethylenediaminetetraacetic acid

G”

Storage modulus

Loss modulus

HA

HepG2

Hyaluronic acid

Hya

Human liver carcinoma cells

$\mathrm{HLH}$

Hyaluronidase

$\mathrm{K}_{d}$

Helix-loop-helix

NMR

Dissociation constant

PEG

Nuclear magnetic resonance

$\mathrm{pI}$

Poly(ethylene glycol)

PMA

Isoelectric point

ROS

Phorbol 12-myristate 13-acetate

SEM

Reactive oxygen species

SPAAC

Scanning electron microscopy

Strain-promoted alkyne-azide cycloaddition

SPPS

TCEP

TEM

Solid phase peptide synthesis

Tris-(2-Carboxyethyl)phosphine

Transmission electron microscopy

$\mathrm{T}_{m}$

Melting temperature

UV-Vis

Ultraviolet-visible

\section{Polypeptide and polymer abbreviations}

\author{
$\mathrm{EI} / \mathrm{KI}$ \\ $\mathrm{pEI}_{4}$ \\ $\mathrm{pEI}_{4} / \mathrm{pKI}_{4}$ \\ $\mathrm{JR}_{2} \mathrm{EC}_{2}$ \\ $\mathrm{p}\left(\mathrm{N}_{3}\right)_{8}$ \\ HA-BCN \\ HA-JR2EK
}

Heterodimer of EI and KI

4-armed star-shaped PEG with terminating EI

Polymeric network of $\mathrm{pEI}_{4}$ and $\mathrm{pKI}_{4}$

Two JR2EC covalent bound through a disulfide

bridge

8-armed star-shaped PEG with terminating $\mathrm{N}_{3}$

$\mathrm{HA}$ functionalized with $\mathrm{BCN}$

HA-BCN functionalized with JR2EK 


\section{Amino acids}

\begin{tabular}{|c|c|c|c|}
\hline Name & Abbreviations & $\begin{array}{l}\mathrm{pK}_{a} \text { (of side } \\
\text { chain group) }\end{array}$ & $\begin{array}{l}\text { Side chain } \\
\text { structure }^{*}\end{array}$ \\
\hline Alanine & Ala or A & & \\
\hline Cysteine & Cys or C & 8.3 & \\
\hline Aspartic acid & Asp or D & 3.9 & \\
\hline Glutamic acid & Glu or E & 4.3 & \\
\hline Phenylalanine & Phe or F & & \\
\hline Glycine & Gly or G & & \\
\hline Histidine & His or $\mathrm{H}$ & 6.0 & \\
\hline Isoleucine & Ile or I & & \\
\hline Lysine & Lys or $\mathrm{K}$ & 10.5 & \\
\hline
\end{tabular}

${ }^{*}$ At $\mathrm{pH} 7$ 


\begin{tabular}{|c|c|c|c|}
\hline Name & Abbreviations & $\begin{array}{l}\mathrm{pK}_{a} \text { (of side } \\
\text { chain group) }\end{array}$ & $\begin{array}{l}\text { Side chain } \\
\text { structure* }^{*}\end{array}$ \\
\hline Leucine & Leu or L & & \\
\hline Methionine & Met or $M$ & & \\
\hline Asparagine & Asn or $\mathrm{N}$ & & \\
\hline Proline & Pro or $\mathrm{P}$ & & \\
\hline Glutamine & Gln or $Q$ & & \\
\hline Arginine & Arg or $\mathrm{R}$ & 12.5 & \\
\hline Serine & Ser or S & & \\
\hline Threonine & Thr or $\mathrm{T}$ & & OH \\
\hline
\end{tabular}

* At pH 7 


\begin{tabular}{|c|c|c|c|}
\hline Name & Abbreviations & $\begin{array}{l}\mathrm{pK}_{a} \text { (of side } \\
\text { chain group) }\end{array}$ & $\begin{array}{l}\text { Side chain } \\
\text { structure }^{*}\end{array}$ \\
\hline Valine & Val or $\mathrm{V}$ & & \\
\hline Tryptophan & Trp or W & & \\
\hline Tyrosine & Tyr or Y & 10.1 & \\
\hline
\end{tabular}

* At pH 7 


\section{Contents}

$\begin{array}{lr}\text { Acknowledgements } & 1\end{array}$

1 Introduction $\quad 3$

1.1 Aim . . . . . . . . . . . . . . . . 5

1.2 Thesis outline ................... 5

2 Self-assembly and supramolecular materials $\quad 7$

2.1 Supramolecular chemistry . . . . . . . . . . . . . 7

2.2 Molecular self-assembly . . . . . . . . . . . . . . 10

2.3 Properties of supramolecular biomaterials . . . . . . . . . . . 12

3 Amino acids, peptides and peptide structure $\mathbf{1 5}$

3.1 Terminology and linear structure of peptides . . . . . . . . . . . . 15

3.2 Folding of peptides . . . . . . . . . . . . . . . 17

3.2 .1 The coiled coil motif . . . . . . . . . . . . . 20

3.2 .2 The helix-loop-helix motif . . . . . . . . . . . 21

4 Polypeptide design and synthesis $\quad 23$

4.1 EKIV-polypeptide system (Paper I) . . . . . . . . . . . . . . . . . 23

4.2 JR-polypeptides . . . . . . . . . . . . . . . . . . . . . . . . . . . . . . . . . . . . . . . . . . 39

4.3 Synthesis of peptides . . . . . . . . . . . . . . . 31

4.3.1 Solid phase peptide synthesis . . . . . . . . . . . 31

4.3 .2 Peptide purification . . . . . . . . . . . . . . 34

4.3 .3 Peptide identification . . . . . . . . . . . . 34

5 Synthesis of polypeptide-hybrids $\quad 35$

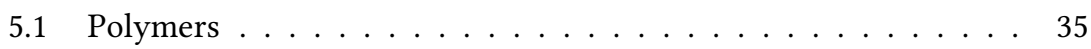

5.1.1 Hyaluronic acid . . . . . . . . . . . . . . 36

5.1 .2 Poly(ethylene glycol) . . . . . . . . . . . . 37

5.2 Modification of polymers and conjugation with peptides . . . . 38

5.2.1 Maleimide-thiol Michael addition . . . . . . . . . . . . . 38

5.2.2 Strain-promoted alkyne-azide 1,3-dipolar cycloaddition . 39 
6 Superstructures, polymeric networks and hydrogels 43

6.1 Terminology .................... 43

6.2 Polymeric networks with the EKIV-polypeptide system (Paper II) 44

6.3 Superstructures with JR2EC $\mathrm{E}_{2}$ and $\mathrm{Zn}^{2+}$ (Paper III) . . . . . . . . . . 49

$6.4 \mathrm{Zn}^{2+}$-responsive hydrogels with HA-JR2EK (Paper IV) . . . . . . . 52

6.5 Hydrogels with HA-JR2EK and $\mathrm{p}\left(\mathrm{N}_{3}\right)_{8}($ Paper V) . . . . . . . . . 56

7 Characterization techniques $\quad \mathbf{6 1}$

7.1 Spectroscopic techniques .................. 61

7.1.1 Absorption spectroscopy . . . . . . . . . . . . 61

7.1.2 Circular dichroism spectroscopy . . . . . . . . . . 62

7.1.3 Dynamic light scattering . . . . . . . . . . . . . . 64

7.1.4 Fluorescence spectroscopy . . . . . . . . . . . 65

7.1.5 Fourier transform nuclear magnetic resonance spectroscopy 66

7.2 Microscopic techniques . . . . . . . . . . . . . . . . 67

7.2.1 Optical- and fluorescence microscopy . . . . . . . . 67

7.2.2 Transmission electron microscopy . . . . . . . . . . 68

7.2.3 Scanning electron microscopy . . . . . . . . . . . . . 69

7.3 Other techniques . . . . . . . . . . . . . . . . 69

7.3.1 Cell viability studies . . . . . . . . . . . . . . 69

7.3 .2 Oscillatory rheology .............. 70

$\begin{array}{lll}8 & \text { Summary of papers } & 73\end{array}$

9 Conclusions and future outlook $\quad 79$

9.1 Conclusions . . . . . . . . . . . . . . . . . . . . 79

9.2 Future outlook . . . . . . . . . . . . . . . 81 


\section{Acknowledgments}

In your hand, or on a monitor in front of you, you have my contribution to science. A contribution created by laughter, tears, eureka moments, $\& \% \Phi !$-moments and "småländsk" stubbornness. However, this thesis could not have been possible without all fantastic people surrounding me. I would like to give my sincere thanks to:

My supervisor Daniel Aili, whose never-ending enthusiasm for science has been and still is truly inspiring.

My two co-supervisors Karin Enander and Staffan Dåmmark for everything from helpful scientific discussions over the years to the proofreading of this thesis.

Current and past Aili-group members for all fruitful, and sometimes scientific, discussions.

Current and past Molecular Physics and Molecular Surface Physics and Nanoscience division members for all discussions and all interesting presentations in our joint biweekly meetings; the sofa is still the best place to sit.

All the members of the Forum Scientium research school, and Stefan Klintström and Charlotte Immerstrand for managing it.

All members of the coffee club "Kaffeklubben" and all "lunch eaters" for making each morning and lunch the highlights of the day.

Linköpings studentspex, and especially all "Dekorare”, for all laughter, weird discussions and late nights at the theatre over the years.

My friends, and especially Eric Elfving for all backpacking adventures, coffee breaks and "surströmming".

My family for always asking me what I actually do but never questioning why I'm doing it.

Amanda for all love and support.

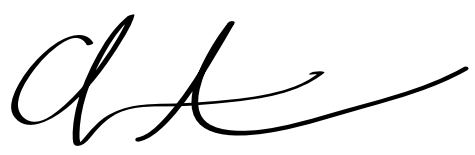

Linköping, November 2016 


\section{Chapter 1}

\section{Introduction}

Equipped with his five senses, man explores the universe around him and calls the adventure Science.

Edwin Powell Hubble

With a rapidly aging population the need for medical devices that can either replace damaged tissues or deliver drugs have never been greater. It is predicted that the global market of biomaterials is going to reach an all-time high of USD $>100$ Billion by the year 2020. ${ }^{1}$ A biomaterial is defined by the European Society for Biomaterials (ESB) as "a material intended to interface with biological systems to evaluate, treat, augment or replace any tissue, organ or function of the body". ${ }^{2}$ Even though it is only recently that biomaterials have gained more attention, humans have already been using them for centuries. As early as in the year $600 \mathrm{AD}$, the Mayan people fashioned nacre teeth from sea shells that could be fully osseointegrated with the jaw. ${ }^{3}$ Other examples include gold sutures used in the ancient Greece to close wounds and contact lenses made out of glass used during the 19th century. ${ }^{3}$ Although many types of materials have been used throughout history, not all of them are compatible with the body and can be highly pathogenic and toxic. ${ }^{4}$ It was not until the 1960 's that the development of materials specifically designed to be used as biomaterial was initiated. When the first generation of biomaterials was developed, the main focus was to match the physiological properties of the tissue to be replaced with minimal toxic response. ${ }^{5}$ Completely or near bioinert materials were the main choice in an attempt to avoid immunological responses. In the 1980's a shift towards more biologically active and resorbable biomaterials was made (second generation biomaterials), contrasting the bioinertness of the first generation. ${ }^{5}$ A biologically active biomaterial can be defined as a material able to elicit a biological response under physiological conditions. ${ }^{6}$ An example of a biologically active material from the 1980 's is synthetic hydroxyapatite ceramic that was successfully used as a porous 
scaffold for regrowth of bone tissue. ${ }^{7-9}$ Resorbable materials from this time era are for instance polymers consisting of e.g. polylactic acid that could be hydrolytically degraded into carbon dioxide and water. The main idea with such resorbable materials was that as the body heals the damaged tissue, the biomaterial is not needed anymore and should degrade and disappear. The resorbable biomaterials were often used as degradable sutures, ${ }^{10,11}$ or as controlled-release drug delivery devices. ${ }^{12,13}$ However, the biomaterials of the second generation were not both biological active and resorbable. It is not until the recent 10-20 years that biomaterials that show both these properties have been developed. The third generation biomaterials are specifically designed to interact and stimulate cells at the molecular level, but are also often degradable ${ }^{5}$ However, the biomaterials of the future will do more than just be bioactive and resorbable. Improved biomimetics, enhanced responsiveness to various stimuli, tunable mechanical and rheological properties are some properties that the future biomaterials will have. During the past few years the term "smart biomaterials" has been used to describe such materials, which also typically are referred to as the fourth generation of biomaterials.

Supramolecular chemistry ${ }^{\mathrm{a}}$ is a great tool to realize the fourth generation of biomaterials. Supramolecular chemistry relies on highly tunable, dynamic and reversible non-covalent interactions. Supramolecular materials can be specifically tailored to respond to numerous physical and chemical stimuli (e.g. temperature, $\mathrm{pH}$, metal chelation), or to distinctly interact with specific biomolecules. The interactions can in turn be used to e.g. precisely control mechanical and rheological properties of hydrogels ${ }^{\mathrm{b}}$ or allow controlled release of drugs. Furthermore, supramolecular interactions allow for convenient fabrication of highly modular biomaterials. Via a mix-and-match approach, various molecular structures (e.g. ECM proteins and polymers, synthetic polymers, therapeutics etc.) can be incorporated into such biomaterials.

Peptides ${ }^{c}$ are excellent supramolecular building blocks to use to create "smart" biomaterials. Peptides can for instance be used to promote cell adhesion, kill microbes or create self-assembling materials. ${ }^{14-16} \quad \beta$-structured peptides have been extensively explored in supramolecular biomaterials. ${ }^{17,18} \beta$-structured peptides have many great advantages for supramolecular biomaterials, such as short peptide sequences and low concentration can often be used to create a hydrogel. However, a major disadvantage with $\beta$-structured peptides is the limited ability to control the assembly process. The disadvantage makes it difficult to use $\beta$-structured peptides to control e.g. the mechanical and rheological properties of hydrogels. In contrast, due to well-established design rules, $\alpha$-helical peptides allow much better control over the assembly. ${ }^{18}$ This thesis describes the development of polypeptide-hybrid biomaterials that are

\footnotetext{
a See chapter 2

${ }^{\mathrm{b}}$ Highly hydrated polymeric networks, see section 6.1

'See chapter 3
} 
based on such $\alpha$-helical peptides. Using dimerizing $\alpha$-helical polypeptides, hydrogels and other structures have been created that respond to different stimuli. The materials can be modularly assembled and are highly tunable, allowing for precise control over the self-assembly process. Consequently, structural and rheological properties of the developed materials can be tuned at the molecular level.

\subsection{Aim}

The overall aim of this thesis has been to develop polypeptides and polypeptide-hybrid biomaterials that can be modularly assembled and where e.g. mechanical and rheological properties can be tuned by means of specific molecular interactions and defined stimuli.

\subsection{Thesis outline}

The thesis describes the development of the polypeptides and the polypeptide-hybrids used in paper I-V, and how these molecules assemble into larger structures with tunable properties. In Chapter 2 the concept of supramolecular chemistry including the process of self-assembly is introduced. Furthermore, properties of supramolecular biomaterials in general are described. Chapter 3 defines what a peptide is and describes how and why peptides have certain structures. Chapter 4 describes in detail the design of the polypeptides used in the thesis. The synthesis and purification strategies for peptides are also described. Chapter $\mathbf{5}$ describes how the polypeptides are conjugated to polymeric backbones to create polypeptide-hybrid biomaterials. Chapter 6 describes in detail how the polypeptides and the polypeptide-hybrids are used to create biomaterials with tunable properties that can be modularly assembled. Chapter 7 presents some of the characterization techniques used in the thesis. Chapter 8 gives a short summary of all papers included in this thesis, listing where essential information regarding the paper can be found throughout the thesis. Chapter 9 summarizes the main conclusions from each paper and gives a brief overall conclusion of the thesis. A future outlook is also presented. At the very end the included papers can be found. 


\title{
Chapter 2
}

\section{Self-assembly and supramolecular materials}

\author{
Chemists have always been in the \\ business of taking atoms and putting \\ them together with other atoms with \\ precisely defined connections.
}

George M. Whitesides

\subsection{Supramolecular chemistry}

Since the dawn of chemistry as a science in the 15-16th century, the main research focus has been on understanding and controlling the covalent nature of molecules. The knowledge obtained over centuries of research enables us today to synthesize molecules that already exist in nature or that until now only have existed in our minds. Over the past five decades, chemists have extended the research focus to also study the interactions between molecules. The existence of intermolecular forces was suggested already in 1873 by Johannes Diderik van der Waals in his doctoral thesis "Over de Continuiteit van den Gas- en Vloeistoftoestand". ${ }^{19}$ However, the scientific field of supramolecular chemistry did not fully emerge until the 1960's. In 1967 Charles J. Pedersen published his seminal work on how crown ethers can be synthesized and how they form stable two-dimensional complexes with metal cations. ${ }^{20}$ Together with Jean-Marie Lehn and Donald Cram, who both expanded upon Pedersens work to include more complex three-dimensional (3D) structures, ${ }^{21,22}$ Pedersen was awarded the Nobel Prize in Chemistry in 1987 " for their development and use of molecules with structure-specific interactions of high selectivity". ${ }^{23}$ In 2016 the Nobel Prize in Chemistry was awarded jointly to Jean-Pierre Sauvage, Sir J. Fraser Stoddart and Bernard L. Feringa "for the design and synthesis of molecular 
machines" ${ }^{24}$ The molecular machines do fully rely on supramolecular interactions, thus showing that supramolecular chemistry still is an important scientific field.

Supramolecular chemistry is often referred to as "the chemistry beyond the molecule", ${ }^{21}$ as two or more molecules, or parts of molecules, interact with each other via non-covalent bonds. It is often described in popular science as "LEGO chemistry" as the molecules, like LEGO-bricks, reversibly can snap together to form larger structures. Furthermore, the scientific field of supramolecular chemistry can broadly be divided into two main categories, host-guest chemistry and self-assembly. The categories are conceptually the same but are differentiated by the size of the interacting species. In host-guest chemistry the interacting species typically differ significantly in size. The host is large enough to enclose the other interacting species (the guest) via supramolecular interactions. Examples include substrates interacting with enzymes and metal-ions interacting with crown ethers. However, if the size of the interacting species is roughly the same the process is conceptually called self-assembly ${ }^{\mathrm{a}}$.

There exists many types of supramolecular interactions, all which can be divided into different categories depending on the nature of the interaction:

Electrostatic interactions: Electrostatic interactions are based on the coulombic attraction between oppositely charged ions or dipoles (Figure 2.1). ${ }^{25}$ An ion-ion interaction can be very strong (200-300 $\left.\mathrm{kJ} \mathrm{mol}^{-1}\right)$, in certain cases even stronger than a covalent bond. It is a non-directional interaction, meaning that the ions have the same interaction energy in all directions and do not have to be aligned in certain directions to maximize their attraction. In comparison, both ion-dipole interactions and dipole-dipole interactions are weaker (50-200 kJ $\mathrm{mol}^{-1}$ and 5-50 $\mathrm{kJ} \mathrm{mol}^{-1}$, respectively) and directional. Although the dipole-dipole interaction is the weakest, this interaction is a useful tool in supramolecular chemistry. Dipole-dipole interactions can be used to align molecules in specific directions, as both dipoles must be perfectly aligned to be able to interact.

Hydrogen bonds: Hydrogen bonding is a specific type of dipole-dipole interaction between a hydrogen atom attached to an electronegative atom and a highly electronegative atom such as nitrogen, oxygen and fluorine. The interaction is directional with an interaction energy ranging from 4 to $120 \mathrm{~kJ}$ $\mathrm{mol}^{-1}$. A common example of hydrogen bonding is the interaction between water molecules. Water molecules tend to form a 3D network of hydrogen bonds as one water molecule is able to form four hydrogen bonds with adjacent water molecules. It is due to this $3 \mathrm{D}$ network that water has the fairly high boiling point of $\approx 100{ }^{\circ} \mathrm{C}$ at sea level in comparison to other group 6A hydrides. $^{26}$

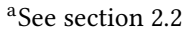




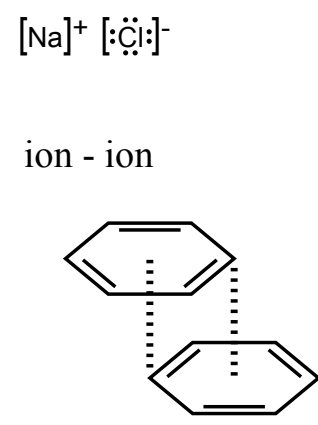

$\pi-\pi$

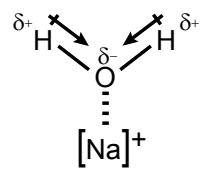

ion - dipole

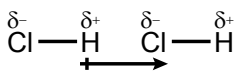

dipole - dipole

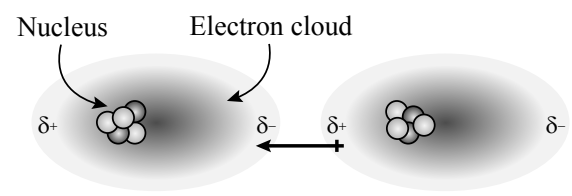

Van der Waals

Figure 2.1: Examples of some supramolecular interactions.

Hydrophobic effects: The hydrophobic effect arises from the tendency of hydrophobic compounds to be excluded from polar solvents such as water (Figure 2.2) ${ }^{25}$ When a hydrophobic compound is added to a water solution, the 3D network of hydrogen bonds will be temporally disrupted to provide space for the hydrophobic compound. However, the water molecules are not be able to form any strong interactions with the hydrophobic compound. Instead the water molecules form a cage-like structure, called a solvent cage, around the hydrophobic compound. ${ }^{27}$ The formation of solvent cages will greatly restrict the mobility of the water molecules, resulting in an unfavorable loss of entropy in the system. To reduce the total number of solvent cages needed, the hydrophobic compounds will aggregate to lower their total surface contact area exposed to the water. The aggregation leads to a net increase in entropy in the system as fewer water molecules are needed to form unfavorable solvent cages. This type of hydrophobic effect is strictly referred to as an entropic hydrophobic interaction. Another type of hydrophobic effect is the enthalpic which occurs when small molecules replaces water within molecular cavities. ${ }^{25}$

$\pi$-interactions: $\pi$-interactions arise from the delocalization of electrons in conjugated systems (e.g. benzene). The "face" side of conjugated system will be negatively charged due to this delocalization. Two main types of $\pi$-interactions are commonly found in supramolecular systems; cation $-\pi$ and $\pi-\pi .^{25}$

van der Waals interactions: Van der Waals interactions arises from temporarily existing dipoles, resulting from fluctuations in the electron distribution of atoms or molecules (Figure 2.1). ${ }^{25}$ Adjacent molecules will align so that a partial positive charge of one molecule is in close proximity to a partial negative charge of another. Van der Waals interactions are non-directional, 


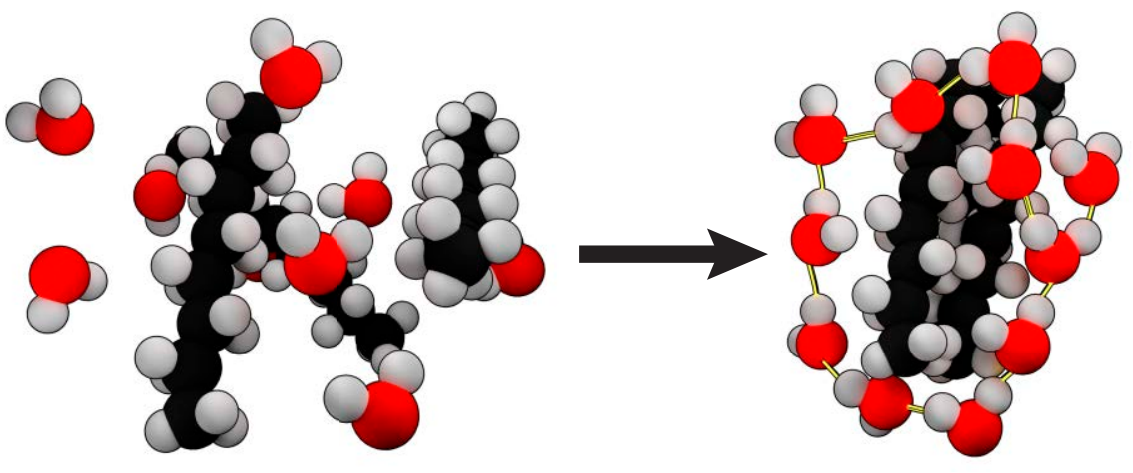

Figure 2.2: As hydrophobic compounds are dissolved in polar solvents, such as water, they are prone to aggregate to minimize their surface contact area with the solvent.

often weak and act only over short distances. However, collectively van der Waals interactions can have large impact on the overall interaction energy between molecules.

The interactions described above are the most common and exploited ones in supramolecular chemistry. However, a single supramolecular interaction is often not enough to bring molecules together. Instead, many individual supramolecular interactions have to collectively act together to form of a stable supramolecule. In addition, the total interaction energy of these collectively acting supramolecular interactions is often greater than the same interactions acting independently of each other. The phenomenon is called positive cooperativity. However, all possible supramolecular interactions on a molecule will not be able to form stabilizing bonds with all types of molecules. The reason for this can be size and shape restrictions, or the actual chemical nature or the interacting species (e.g. positively or negatively charged). The molecules must be complementary with respect to both structure and interactions. A high degree of complementarity will exclude some molecules and only allow some to interact, resulting in selectivity.

\subsection{Molecular self-assembly}

Molecular self-assembly is the spontaneous and reversible association of two or more molecular components to form ordered supramolecular structures without human intervention. ${ }^{28}$ Self-assembly can be divided into inter- and intramolecular self-assembly. Intermolecular self-assembly occurs between separate molecules whereas intramolecular self-assembly occurs between components within a single molecule. Both types lead to a more ordered state 
for the molecule(s) and are important in e.g. folding of polypeptides ${ }^{\mathrm{b}}$.

Self-assembly is a dynamic process that allow non-covalently interacting species to reach a more thermodynamically favorable state. The process generally leads to an equilibrium state, although many non-equilibrium processes do exist (e.g. living systems). ${ }^{29}$ When a process is in equilibrium it has reached a state where the concentrations of reactants and products are not changing over time. However, the reached state may not necessary be the most thermodynamically stable state (lowest Gibbs energy). A supramolecular product can become kinetically trapped and unable to proceed to a more thermodynamically favorable state. In statistically energy landscape theory this is visualized as a local minimum that has a higher energy level as compared to the global minimum (thermodynamic stable state) (Figure 2.3). ${ }^{30}$

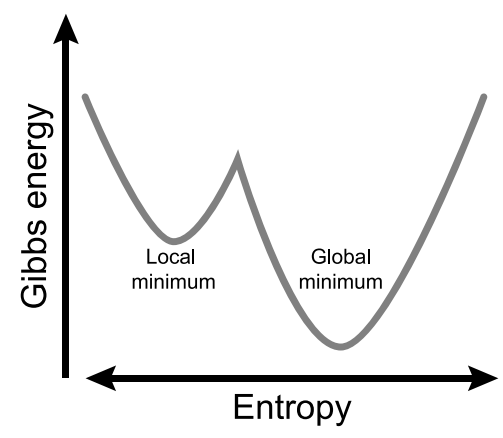

Figure 2.3: Generic 2D energy landscape visualizing the difference between a local and a global energy minimum.

The ratio between the concentrations of supramolecular products and reactants in equilibrium is often referred to as the binding constant or the association constant $\left(\mathrm{K}_{\mathrm{a}}\right)$. For peptides and proteins it is more common to use the dissociation constant $\left(\mathrm{K}_{\mathrm{d}}\right)$, which is the reciprocal of $\mathrm{K}_{\mathrm{a}}$. For a two-component system binding in 1:1 ratio at equilibrium, $\mathrm{K}_{\mathrm{d}}$ will be

$$
K_{d} \equiv \frac{1}{K_{a}}=\frac{[A][B]}{[A B]}
$$

where [A] and [B] are the supramolecular reactants and [AB] is the supramolecular product. ${ }^{31}$ The $K_{d}$ has units of $M$ where a small value indicates a high binding affinity. The $K_{d}$ of a binding event can be experimentally determined using several different methods. As an example, in paper I the different $K_{d}$-values of the heterodimerized EKIV-polypeptides ${ }^{c}$ were determined using circular dichroism spectroscopy $(\mathrm{CD})^{\mathrm{d}}$. Other common techniques used to

\footnotetext{
${ }^{\mathrm{b}}$ See chapter 3 and chapter 4

'See chapter 4.1

${ }^{\mathrm{d}}$ See chapter 7.1.2
} 
estimate binding constants are e.g. ELISA, ${ }^{32}$ surface plasmon resonance, ${ }^{33}$ and isothermal titration calorimetry. ${ }^{34}$

Selectivity is the ability of molecules to discriminate between many possible binding partners. Two main types of selectivity are common in supramolecular systems: kinetic- and thermodynamic selectivity. ${ }^{25}$ Kinetic selectivity is often found in enzyme-based processes where the substrate that has the overall shortest reaction rate with an enzyme (i.e. association, convertion and dissociation) will give more products. Thermodynamic selectivity does instead only rely on the binding affinity. Supramolecular products with lower $\mathrm{K}_{\mathrm{d}}$ will form to a greater extent compared to supramolecular products with a higher $\mathrm{K}_{\mathrm{d}}$. In systems with many potential binding partners, thermodynamic selectivity results in a phenomenon called self-sorting (Figure 2.4). ${ }^{35}$ Depending on which final supramolecular products that are formed, self-sorting can be divided into different categories. In narcissistic self-sorting molecules form supramolecular products with like molecules. In contrast, if molecules form supramolecular products with other molecules it is called social self-sorting. Furthermore, self-sorting can either be nonintegrative or integrative. The polypeptides developed and used in paper I and II is an example of social nonintegrative self-sorting. The polypeptides and their ability to self-sort will be discussed in more detail in section 4.1.

\subsection{Properties of supramolecular biomaterials}

Biomaterials that self-assemble via supramolecular interactions are finding more and more applications in medicine? In comparison to materials comprised only of covalently bound molecules, supramolecular interactions can give materials properties that are not easily achieved otherwise. Supramolecular materials are highly modular in the sense that different materials with the same or a complimentary self-assembly motif can be mix-and-matched. The modularity allows for a precise control over e.g. the composition and functionality of the final material. Supramolecular materials can also show tunable mechanical and structural properties. Although covalent bonds can be used to tune mechanical stabilities by e.g. varying the crosslinking density, the mechanical properties cannot easily be altered after the fabrication of the material. Furthermore, if a covalent bond is disrupted by external forces such as mechanical stress it cannot be reformed, which may result in loss of function of the material. In contrast, supramolecular interactions allow the mechanical properties to be changed at any time in the fabrication of the material or during use. The mechanical properties of supramolecular materials can be tuned by either varying the concentration of the self-assembling moieties or the type and affinity of the supramolecular interactions used. In addition, the dynamic properties of supramolecular bonds allow them to be reformed if disrupted. This enables development of sheer-thinning and self-healing materials that for instance can be reformed when exposed to disrupted forces without loss of 
function. ${ }^{36}$ Furthermore, supramolecular biomaterials can respond to different types of external stimuli. Examples of such external stimuli are changes in ionic strength, $\mathrm{pH}$ and temperature. ${ }^{37-43}$ By exposing a supramolecular material to such stimuli it is possible to, in real-time, change properties of the material.

Commonly used supramolecular moieties in supramolecular biomaterials are those based on host-guest interactions (e.g. cyclodextrins), ${ }^{44-46}$ metal-ligand coordination, ${ }^{47-49}$ and polyvalent hydrogen bonding (e.g. DNA).$^{50}$ In this thesis, polypeptides have been used as the supramolecular moiety. Polypeptides can be designed to feature various supramolecular interactions at very precise positions, making them highly modular supramolecular moieties. The structure of polypeptides is discussed in the next chapter.

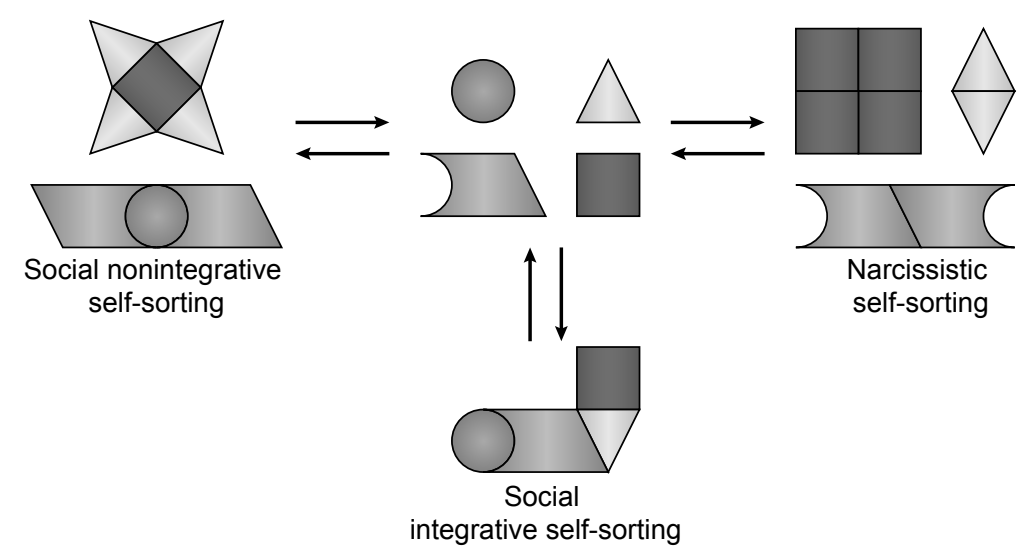

Figure 2.4: Schematic representation of different types of self-sorting. 
CHAPTER 2. SELF-ASSEMBLY AND SUPRAMOLECULAR MATERIALS 


\section{Chapter 3}

\section{Amino acids, peptides and peptide structure}

I caught a cold and after a day or two in bed of reading science fiction and detective stories, I got tired of that, and thought, why don't I discover the alpha helix?

Linus Pauling

This chapter will describe what peptides are, what peptides consist of and give some terminology commonly used when describing peptides. Furthermore, the folding of peptides will be described, including the structures and the folding motifs of the peptides used in this thesis.

\subsection{Terminology and linear structure of peptides}

Peptides (and proteins) consist of small monomeric units that are covalently linked into chains. The monomeric unit is called amino acid and consists of a carbon atom that is covalent bound to both a carboxyl group and an amine (Figure 3.1). The carbon atom between the two functional groups is denoted $\mathrm{C}_{\alpha}$. From $\mathrm{C}_{\alpha}$ other carbon atoms can be covalent bound, denoted $\mathrm{C}_{\beta}, \mathrm{C}_{\gamma}$ etc. in successive order of the Greek alphabet. The chain bound to the $\mathrm{C}_{\alpha}$ is called the side chain of the amino acid. Although more than 500 amino acids are known to exist in nature to date, ${ }^{51}$ only 20 of these are usually found in proteins. The structure of these 20 amino acids side chains is summarized in the front matter of this thesis. Furthermore, amino acids can be divided into various categories depending on the characteristics of the side chain. The side chain can be hydrophobic, mostly consisting of hydrocarbon groups (Ala, Phe, Ile, Leu, Met, 
Trp, Tyr and Val). They can be positively charged (Arg, His and Lys), negatively charged (Asp and Glu), or uncharged but polar (Asn, Gln, Ser and Thr). Then there are three more that can be considered special cases. The side chain of Cysteine (Cys) terminates with a thiol group. The Cys thiol can be covalently bound to other Cys thiols, crosslinking two Cys into a Cystine. The crosslink is often called a disulfide bridge, a terminology that for instance is used in paper III. In addition, Glycine (Gly) does not have a side chain at all. In contrast, the side chain of proline (Pro) is connected directly to the $\mathrm{C}_{\alpha}$-amino group.

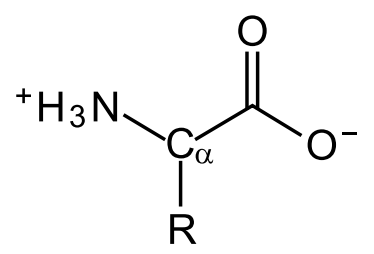

Figure 3.1: The chemical structure of an amino acid. A carboxyl group and an amine group are covalently bound to $\mathrm{C}_{\alpha}$. Different types of amino acids have different side chains ( $\mathrm{R}$ in the figure) bound to the $\mathrm{C}_{\alpha}$.

Peptides are strictly defined as polyamides due to the bonds in the polymer chain. By covalently linking the amino group of one amino acid with the carboxyl group of another, a peptide is formed (Figure 3.2). The amide linkage between amino acids is commonly called a peptide bond and each amino acid in the peptide is called an amino acid residue. Depending on the number of amino acid residues in the chain, various prefixes are used. A peptide containing two amino acid residues is called a dipeptide, three amino acids a tripeptide, four amino acids a tetrapeptide etc. An oligopeptide refers to peptide consisting of $\approx 2-10$ amino acid residues whereas a longer peptide is called a polypeptide. When the length of a polypeptide exceeds $\approx 50$ amino acid residues it is more common to refer to it as a protein, at least if it has biological origin.<smiles>[R]C(N)C(=O)O[CH2+]</smiles><smiles>C[O-]</smiles><smiles>[R]C(N)C(=O)NC([R2])C(=O)O</smiles>

Figure 3.2: Peptide bond formation by a condensation reaction.

\footnotetext{
aThe term "polymer" is defined in section 5.1
} 
Peptides are most often linear polymers, although peptides can be found as cyclic polymers. One end of a linear peptide terminates with an amino acid residue that has a free amino group whereas the other end terminates with an amino acid residue that has a free carboxyl group. These ends are called the $\mathrm{N}$ and the C-terminus, respectively. By convention, peptide structures are written with the N-terminus to the left and the C-terminus to the right. ${ }^{52}$ As an example, the EV-polypeptide used in paper I and paper II is by definition called

Acetylglutamylvalylserylalanylleucinylglutamyllysinylglutamylvalylserylalanylleucinylglutamyllysinylglutamylasparaginylserylalanylleucinylglutamyltryptophanylglutamylvalylserylalanylleucinylglutamyllysinylamide.

For simplification, it is more common to use the three- or one-letter abbreviation of the amino acids when typing out the sequence of a peptide. For the EV-polypeptide, with an acetylated N-terminus and an amidated C-terminus, the one-letter abbreviation would thus be

\section{Ac-EVSALEKEVSALEKENSALEWEVSALEK-NH ${ }_{2}$}

\subsection{Folding of peptides}

The specific linear sequence of amino acids in a peptide is called the primary structure. Certain orders of amino acid residues can allow a peptide to fold into a local 3D structure called a secondary structure. As a peptide folds into a secondary structure, some folds will be prevented due to geometric restrictions of the peptide bond. The peptide bond has a double-bond character due to resonance contributions from the adjacent double-bonded oxygen atom. ${ }^{53}$ Rotation about the peptide bond will thus be markedly hindered and make the six atoms that constitutes the peptide bond to be planar (Figure 3.3). This will restrict the possible rotations of the peptide backbone to about the $\mathrm{C}_{\alpha}-\mathrm{C}(\phi)$ and $\mathrm{N}-\mathrm{C}_{\alpha}(\psi)$ bonds.

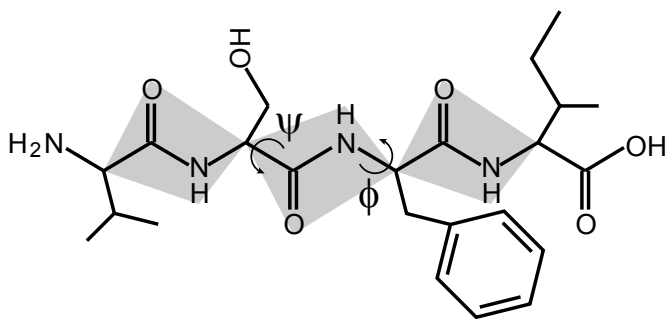

Figure 3.3: Rotation of the polymer backbone is only permitted about the $\mathrm{C}_{\alpha}-\mathrm{C}(\phi)$ and $\mathrm{N}-\mathrm{C}_{\alpha}(\psi)$ bonds due to geometrical restrictions of the peptide bond. 
In 1969 the American molecular biologist Cyrus Levinthal made a thought experiment, where he noted that a protein could have an almost endless number of possible conformations. ${ }^{54}$ To exemplify, if each $\phi-$ and $\psi$ bond was restricted to only three states, then a 100 amino acid residue protein could have $3^{198} \approx 3 \times 10^{98}$ possible conformations. If such a protein would randomly try to find its native state (proper folded structure) at a rate of $10^{10}$ folds per second, it would take more than $10^{75}$ years. However, proteins can fold into its native state within seconds or less. This contradiction in time of folding is often referred to as the Levinthal's paradox. Luckily, the folding of proteins is far from completely random. Supramolecular interactions, such as hydrogen bonding and hydrophobic effects, between amino acids will selectively allow and disfavor certain conformations. By restricting the total amount of possible conformations, a folding pathway is created that guides the protein to its native state. The energy landscape of the folding can be depicted as a rugged funnel (Figure 3.4). ${ }^{30,55}$ The rugged structure is due to local minima where the protein will transiently reside during the folding process. Unless the protein gets kinetically trapped in such a local minimum, it will eventually fold into its native state, reaching the lowest possible energy and the most ordered (low entropy) state. However, sometimes an even lower energy state than the native state is possible. Example of this is amyloid formation caused by protein misfolding. ${ }^{56}$

The thermodynamics for folding of peptides is as follows. In the initial phase the peptides will exist in multiple random conformations, often called the random coil conformation. Due to the great number of possible conformations, the system will have high conformational entropy. Upon folding the number of possible conformations will decrease, thus decreasing the entropy. However, the thermodynamic equation for change in Gibbs energy

$$
\Delta G=\Delta H-T \Delta S
$$

where $\Delta \mathrm{G}$ is the Gibbs energy, $\Delta \mathrm{H}$ is the enthalpy and $\Delta \mathrm{S}$ is the entropy, implies that a decrease in entropy leads to $\Delta G>0$, thus making the process non-spontaneous. However, upon folding the peptide will form stabilizing interactions such as hydrogen bonds, salt bridges and van der Waals interactions. Formation of supramolecular bonds releases energy to the system, making $\Delta \mathrm{H}$ negative. The enthalpic contribution will be greater than the entropic contribution upon folding, making $\Delta G<0$ and thus the folding process spontaneous. In addition, the surrounding solvent do also influences the folding of peptides. The hydrophobic effect causes hydrophobic amino acid residues to aggregate to minimize the total amount of water molecules needed to form solvent cages. The hydrophobic effect will thus increase the entropy of the system by releasing water molecules that previously were used to form solvent cages. 


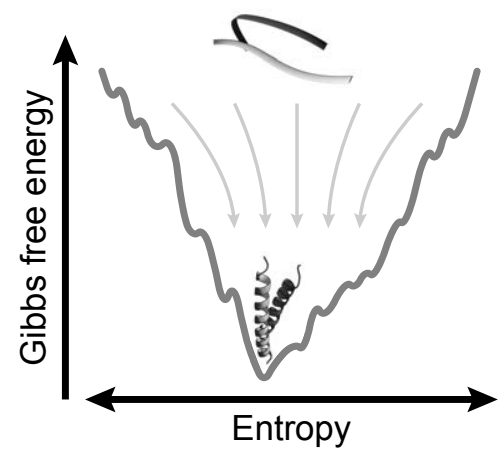

Figure 3.4: A visualization of a $2 \mathrm{D}$ energy landscape for the folding process of two peptides into an more ordered structure.

Upon folding, certain $\phi$ - and $\psi$ dihedral angles will be favored to form stable secondary structures. Two common secondary structures are the $\beta$-strand and the $\alpha$-helix. $\beta$-strand forming peptides are to date the most commonly used peptides in supramolecular biomaterials. ${ }^{17,18}$ Examples include, but are not limited to, the peptide amphiphiles developed by the Tirell group and later refined by the Stupp group, ${ }^{57,58}$ the MAX-peptides developed by the Schneider group, ${ }^{59,60}$ the commercially available PuraMatrix based on the EAK16-polypeptide developed by Zhang et al., ${ }^{61}$ the small Fmoc-FF hydrogelators, ${ }^{62}$ and many naturally occurring proteins such as spider silk. ${ }^{63}$ To stabilize a $\beta$-strand, two or more $\beta$-strands must be aligned side-by-side, forming a $\beta$-sheet (Figure 3.5a). The reason for this is the backbone hydrogen bonding of the $\mathrm{C}=\mathrm{O}$ and $\mathrm{NH}$ groups. In a $\beta$-strand configuration, the $\mathrm{C}=\mathrm{O}$ and $\mathrm{NH}$ groups of the backbone will be directed approximately perpendicular from the backbone direction. As the hydrogen bonding occur on both sides of the $\beta$-strand the formation of $\beta$-sheets does often lead to the formation of long, one-dimensional fibers. The spontaneous formation of fibers is one of the main reasons why $\beta$-structured peptides often are used to create hydrogels.

As mentioned previously, another common secondary structure is the $\alpha$-helix. In the early 1930's William Astbury discovered that upon stretching of moist wool and hair fibers, drastic changes occurred in the X-ray fiber diffraction pattern. ${ }^{64}$ The data suggested that keratin, which wool and hair are made of, in its unstretched state has a "coiled" structure with a characteristic repeat of 0.51 $\mathrm{nm}$. Astbury denoted this as the $\alpha$-form, a terminology that was kept when Linus Pauling et al. proposed the actual structure of the $\alpha$-helix in $1951{ }^{65}$ The $\alpha$-helix, also called $3.6_{13}$-helix, is the most abundant helical conformation of proteins. ${ }^{66}$ The peptide backbone forms a right-handed helical structure via stabilizing intramolecular hydrogen bonds (Figure 3.5b). ${ }^{18}$ Each NH group in the backbone forms a hydrogen bond with a backbone $\mathrm{C}=\mathrm{O}$ four positions earlier in the amino acid sequence. To allow this conformation, the backbone will rotate 
a)

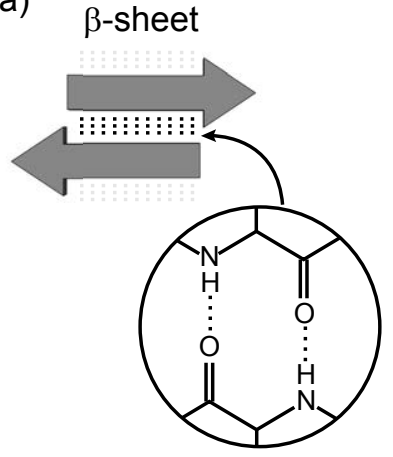

b)

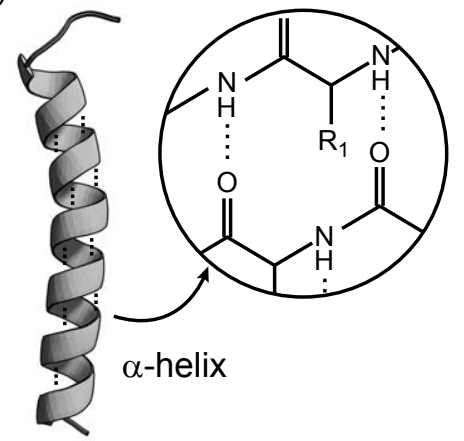

Figure 3.5: The hydrogen bonding nature of the a) $\beta$-sheet and b) $\alpha$-helix. Hydrogen bond formation in $\beta$-sheets requires several $\beta$-strands whereas an $\alpha$-helix have an internal hydrogen bond network.

the dihedral angles to $\phi \approx-64^{\circ}$ and $\psi \approx-41^{\circ} .{ }^{53}$ Each amino acid residue will contribute to a $100^{\circ}$ turn to the helix, making a full turn consisting of 3.6 residues. Furthermore, all backbone $\mathrm{C}=\mathrm{O}$ will point toward the $\mathrm{C}$-terminus, which leads to an overall dipole moment in the helix. In addition, favorable van der Waals interactions "fills" the interior space of the helix with even more stabilizing interactions. ${ }^{67}$

Even though $\beta$-strands readily are used in peptide-based biomaterials, the associated hydrogen bond network makes it difficult to control their assembly. ${ }^{18}$ Since the $\alpha$-helix possesses an internal hydrogen bond network it can be seen as a more discrete building block in comparison to the $\beta$-strand. Furthermore, by employing well-established sequence-to-structure relationship rules it is possible to design $\alpha$-helices that allow for precise self-assembly and oligomerization. ${ }^{68}$ Using such rules, $\alpha$-helical polypeptides have been used to create well-defined fibers, ${ }^{69-73}$ and hydrogels, ${ }^{70,74,75}$ and even as well-defined structures as nanometer-sized tetrahedons. ${ }^{76}$ Two $\alpha$-helical folding motifs that have been used in this thesis are the coiled coil motif and the helix-loop-helix motif.

\subsubsection{The coiled coil motif}

The coiled coil is a common structural motif where two or more $\alpha$-helices wrap around each other (oligomerization) to form a stable supramolecular structure. The existence of the coiled coil motif was proposed independently by Linus Pauling and Francis Crick in 1953, ${ }^{77,78}$ only two years after Pauling et al. had proposed the structure of the $\alpha$-helix. ${ }^{65}$ Coiled coil polypeptides do often consist of a heptad amino acid repeat pattern of HPPHPPP where $\mathbf{H}$ are 
hydrophobic and $\mathbf{P}$ are polar amino acid residues, respectively. ${ }^{68}$ A more general used heptad repeat sequence terminology is (abcdefg) $n_{n}$ where $a$ and $d$ corresponds to the hydrophobic amino acid residues in the polypeptide and $n$ is the total number of heptad repeats. The heptad repeat is often depicted in a helical wheel diagram to visualize the relative position of each amino acid residue in the heptad repeat when the polypeptide is folded (Figure 3.6a). Furthermore, a coiled coil polypeptide is amphipathic when folded. When the polypeptide folds a hydrophobic strip is formed along one side of the $\alpha$-helix. To maximize favorable interactions with other $\alpha$-helices, the average turn distance between an $a$ and a $d$ residue will be 3.5 residues, compared to a regular $\alpha$-helix with 3.6 residues per turn. To reduce the turn to 3.5 amino acid residues, the $\alpha$-helices must align their hydrophobic strips and wrap around one another in the opposite direction as the polypeptide backbone to form a "superhelix" (Figure 3.6b). The alignment of the hydrophobic strips results in a hydrophobic core between the $\alpha$-helices that is thermodynamically favorable due to the hydrophobic effect. The hydrophobic effect is the main driving force for the self-assembly of coiled coils. ${ }^{79}$ The remaining residues at $b, c, e, f$ and $g$ positions can also aid in the formation and stabilization of a coiled coil. Such stabilization will be discussed and exemplified more in detail in chapter 4 .

a)

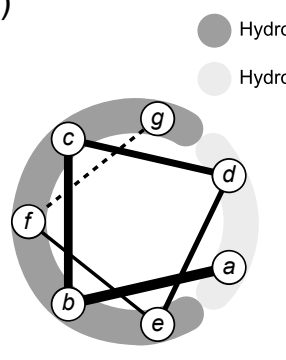

Hydrophilic

drophobic

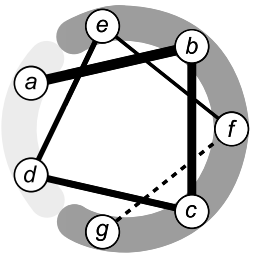

b)

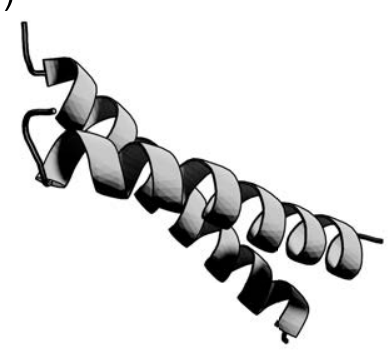

Figure 3.6: a) Helical wheel diagram showing the amphipathic nature of coiled coil forming $\alpha$-helices. b) Cartoon representation of a dimeric coiled coil structure.

\subsubsection{The helix-loop-helix motif}

The helix-loop-helix (HLH) motif consists of two $\alpha$-helices that are joined together by a short loop region (Figure 3.7). The HLH is a structural motif that is commonly found in transcriptional regulatory proteins that are important in a variety of developmental processes.$^{80}$ For instance, in humans HLH containing proteins are involved in the development of the endocrine pancreas and the neocortex. ${ }^{81,82}$ Furthermore, the HLH motif can be found in both a monomericand in an oligomeric state. ${ }^{80}$ Similar as the coiled coil motif presented in subsection 3.2.1, dimerization of two HLH motifs leads to the formation of a hydrophobic core. The HLH can dimerize either in a parallel, an anti-parallel or 
a bisecting $\mathrm{U}$ arrangement, ${ }^{83}$ all leading to the formation of a four-helix bundle. The polypeptide JR2E used in paper III, IV and V is an example of a HLH motif that folds into an anti-parallel four-helix bundle. The JR2E polypeptide will be described more in detail in section 4.2 .

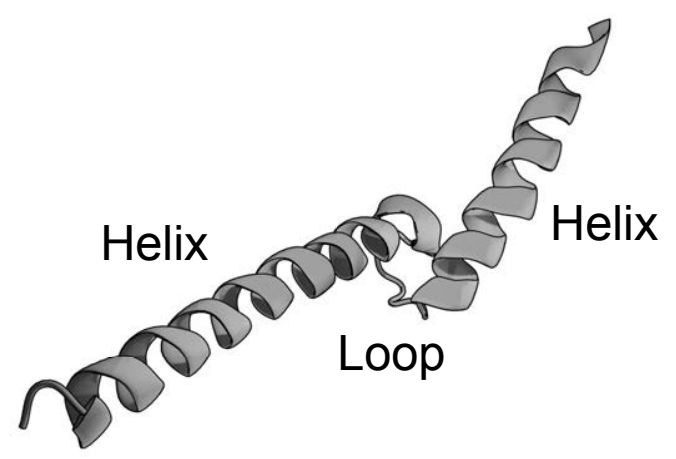

Figure 3.7: A part of the DNA-binding MyoD basic-HLH domain (PDB entry 1MDY), showing the structure of a helix-loop-helix motif. 


\section{Chapter 4}

\section{Polypeptide design and synthesis}

Innovate, automate - or evaporate!

Robert Bruce Merrifield

In this chapter the polypeptides used in this thesis will be presented. In addition, the main results from paper I will be highlighted in section 4.1. Lastly, strategies for synthesis and purification of peptides will be described.

\subsection{EKIV-polypeptide system (Paper I)}

The EKIV-polypeptide system was developed in this thesis and was used in paper I and II. The EKIV-polypeptide system consists of four 28 amino acid residue polypeptides, designed to fold into parallel, heterodimeric $\alpha$-helical coiled coils (Figure 4.1 and table 4.1).

\begin{tabular}{|c|cccc|}
\hline \multicolumn{5}{|c|}{ Sequences and heptad register } \\
\hline & gabcdef & gabcdef & gabcdef & gabcdef \\
\hline EI & EIAALEK & EIAALEK & ENAALEW & EIAALEK \\
KI & KIAALKE & KIAALKE & KNAALKW & KIAALKE \\
EV & EVSALEK & EVSALEK & ENSALEW & EVSALEK \\
KV & KVSALKE & KVSALKE & KNSALKW & KVSALKE \\
\hline
\end{tabular}

Table 4.1: The primary structure of the EKIV-polypeptides.

As discussed in chapter 3, coiled coils are often amphipathic and have the repeating heptad pattern HPPHPPP of hydrophobic and polar amino acid residues. The pattern was the starting point for the design of each 

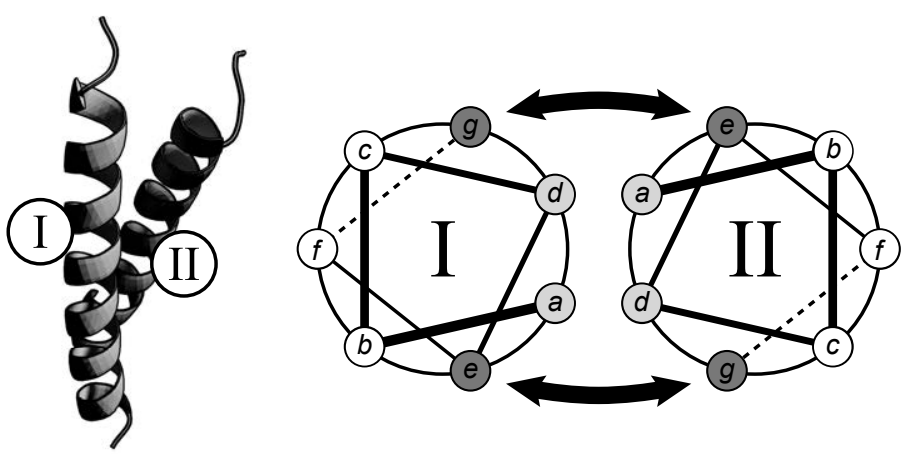

Figure 4.1: When two complementary EKIV-polypeptides are mixed, a spontaneous selfassembly process leads to formation of stable parallel heterodimers. A hydrophobic core forms with the amino acid residues at $a$ and $d$ positions, and stabilizing electrostatic interactions exist between $e$ and $g$ positions.

EKIV-polypeptide. Furthermore, the sequences were inspired by polypeptides originally de novo designed in the groups of Hodges ${ }^{79,84}$ and Woolfson. ${ }^{85}$ However, specific modifications were made with the aim to obtain two main sets of complementary polypeptides with large differences in affinities for dimerization.

The $a$ and $d$ positions consist mainly of hydrophobic amino acid residues. At $d$ positions leucine (Leu, L) was used whereas at the $a$ positions a $\beta$-branched amino acid was used; isoleucine (Ile, I) in EI and KI, and valine (Val, V) in EV and KV. In the third heptad at position $a$ an asparagine (Asn, N) was incorporated to promote formation of parallel, dimeric coiled coils. ${ }^{85,86}$ To enable formation of the hydrophobic core the two Asn must pair up (Asn-Asn') to form a stabilizing hydrogen bond (see Figure 3 in paper I). In addition, the asymmetric positioning of the Asn residue in the polypeptide sequences promotes formation of in-register structures over out-of-register structures. Furthermore, around the hydrophobic core, at $e$ and gpositions, charged amino acid residues were incorporated. EI an EV have negatively charged glutamic acids (Glu, E) at these positions whereas KI and KV have positively charged lysines (Lys, K) at these positions. The charge difference will not only reduce homodimerization by charge repulsion, but also promote heterodimerization by charge attraction when two complementary polypeptides are mixed. The charge attraction between Glu-Lys' is called a salt bridge and is a combination of an electrostatic interaction and a hydrogen bond ${ }^{87}$ In addition, at the $b$ positions alanine (Ala, A) or serine (Ser, S) were used. Ala has a higher helical propensity compared to Ser, hence the polypeptides EI and KI with Ala in these positions will be more prone to fold into $\alpha$-helices. ${ }^{88}$ At position $c$ all polypeptides have an Ala. At $f$ positions mostly charged residues were used that are of the opposite charge as those at $e$ and $g$ positions. This to increase the water 
solubility of the polypeptides over a larger $\mathrm{pH}$ range. However, in the third heptad at position $f$ a tryptophan (Trp, W) was incorporated as a chromophore to enable spectroscopical determination of polypeptide concentration ${ }^{\mathrm{a}}$.

In paper I the design of the polypeptides was devised and evaluated. Each individual polypeptide existed mainly as random coils at $\mathrm{pH} 7$, as determined by $\mathrm{CD}$ (Figure 4.2a). However, EI and KI did have some minor tendencies to form homomeric coiled coil structures, seen as the small shift of the negative band in the CD spectra from $198 \mathrm{~nm}$ to 201-202 nm. The slightly higher tendency for EI and KI to form homomeric structures is due to the incorporation of Ile at the $a$ positions. Ile has an extra methylene group compared to Val which leads to a more pronounced hydrophobic effect. ${ }^{79}$ Furthermore, it was also noted that the KI homomers displayed slightly higher thermal stability compared to the EI homomers (Figure 4.2b). The reason for this is the different amino acid residues at $e$ and $g$ positions. ${ }^{89}$ The conformational flexibility of the Lys side chain allows the $\epsilon$-amine groups between two Lys-Lys' to be separated enough to overcome some of the charge repulsion and thus allow formation of homomeric coiled coils. In contrast, the shorter and less flexible side chain of Glu cannot separate the $\gamma$-carboxyls to the same extent. Glu is thus more effective in preventing homomeric EI structures to be formed in comparison to Lys in KI. However, the homomeric structures of EI and KI were not very stable at $\mathrm{pH} 7$ as slightly elevated temperatures could abolish them completely (Figure 4.2b). In addition, by adjusting the $\mathrm{pH}$ closer, or beyond, the isoelectric point (pI) of each polypeptide it was possible to form more stable homomeric structures, as shown in paper I and II. The reason for the increased stability closer to the $\mathrm{pI}$ is due to formation of stabilizing hydrogen bonds as the side chain of Glu and Lys is protonated and deprotonated, respectively (Figure 4.3).

a)

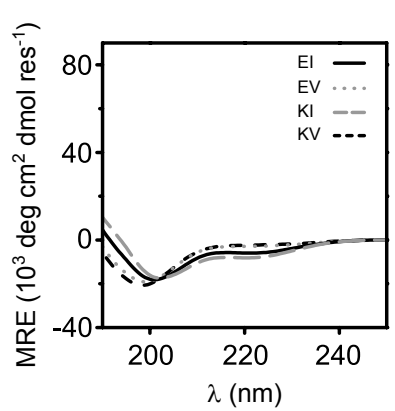

b)

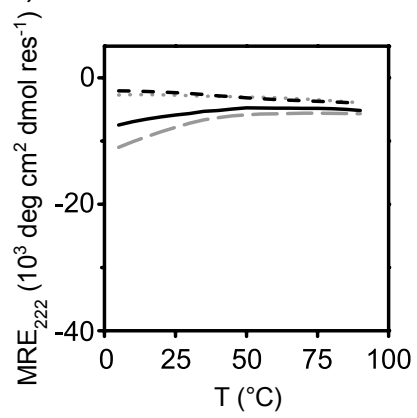

Figure 4.2: (a) CD spectra of each individual polypeptide at $20{ }^{\circ} \mathrm{C}$. (b) Thermal denaturation curves of individual polypeptides.

\footnotetext{
${ }^{\text {a}}$ For details, see chapter 7.1.1
} 
<smiles>[R]C1=[O+][OH+][OH+][OH+]C([R])O1</smiles>

Glu - Glu'<smiles></smiles><smiles>[AsH2][AsH2]</smiles>

Figure 4.3: As the Glu and the Lys side chain becomes protonated and deprotonated, respectively, it can form stabilizing hydrogen bonds. Due to the directional constraints of hydrogen bonding, the Lys-Lys' interaction will at most form one hydrogen bond whereas the Glu-Glú interaction can consist of two hydrogen bonds, making the later more stable.

The two main sets of complementary polypeptides consist of the combination of $\mathrm{EI} / \mathrm{KI}$ and $\mathrm{EV} / \mathrm{KV}$. However, it is also possible to combine these two main sets to form two other subsets; EI/KV and EV/KI. As shown in paper I, all combinations did adopt an $\alpha$-helical conformation (Figure 4.4a). Comparing the $\frac{\mathrm{MRE}_{222}}{\mathrm{MRE}_{208}}$ ratio between the different combinations, ${ }^{90} \mathrm{EI} / \mathrm{KI}$ formed the most well-defined $\alpha$-helical and coiled coil structure with a ratio of 1.01 whereas $\mathrm{EV} / \mathrm{KV}$ formed the least well-defined structure with a ratio of 0.85 . The EI/KV and EV/KI had ratios 0.97 and 0.94 , respectively. Furthermore, thermal denaturation experiments showed that the different combinations had very different thermal stabilities with $\mathrm{T}_{m}$ ranging from 37 to $87^{\circ} \mathrm{C}$ (Figure $4.4 \mathrm{~b}$ ). The results show that the small differences between Ile- and Val-containing peptides give rise to major differences in terms of structural conformation and stability. The CD-characterization data is summarized in table 4.2.

a)

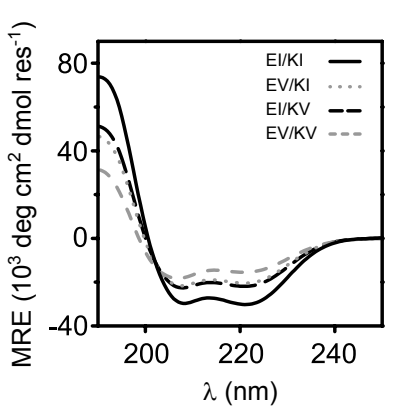

b)

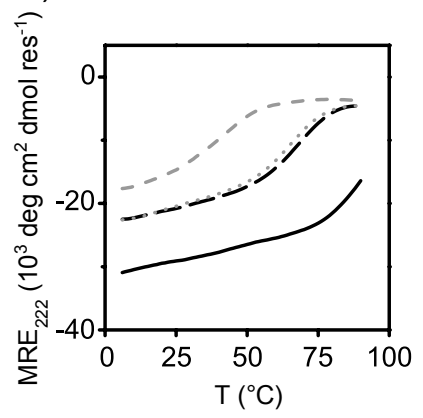

Figure 4.4: (a) CD spectra of combined polypeptides at $20{ }^{\circ} \mathrm{C}$. (b) Thermal denaturation curves of combined polypeptides.

The $\mathrm{K}_{\mathrm{d}}$ of each heterodimer was estimated using a method described by Marky and Breslauer. ${ }^{31}$ By running thermal denaturation experiments at several 


\begin{tabular}{lcccc} 
Polypeptide(s) & MRE $_{222}^{*}$ & $\overline{\mathbf{M R E}}_{222}$ & $\mathbf{T}_{m}\left({ }^{\circ} \mathbf{C}\right)$ & $\mathrm{K}_{\mathrm{d}}(\mathrm{M})$ \\
\hline $\mathrm{MI}$ & -5.9 & 0.56 & $<20$ & \\
$\mathrm{EV}$ & -4.0 & 0.36 & $<5$ & \\
$\mathrm{KI}$ & -8.0 & 0.63 & $<20$ & \\
$\mathrm{KV}$ & -2.4 & 0.29 & $<5$ & \\
$\mathrm{EI} / \mathrm{KI}$ & -30.1 & 1.01 & 87 & $<1.0 \times 10^{-10}$ \\
$\mathrm{EV} / \mathrm{KI}$ & -20.4 & 0.94 & 62 & $8.5 \times 10^{-8}$ \\
$\mathrm{EI} / \mathrm{KV}$ & -21.8 & 0.97 & 64 & $7.2 \times 10^{-8}$ \\
$\mathrm{EV} / \mathrm{KV}$ & -15.4 & 0.85 & 37 & $1.4 \times 10^{-6}$ \\
\hline$* 10^{3} \mathrm{deg} \mathrm{cm} \mathrm{dmol} \mathrm{res}^{-1}$ & & & &
\end{tabular}

Table 4.2: $\mathrm{MRE}_{222}, \frac{\mathrm{MRE}_{222}}{\mathrm{MRE}_{208}}$, melting temperature $\left(\mathrm{T}_{m}\right)$, and $\mathrm{K}_{\mathrm{d}}$ of individual and combined polypeptides at $\mathrm{pH} 7$ and $20{ }^{\circ} \mathrm{C}$. The total polypeptide concentration is $50 \mu \mathrm{M}$.

concentrations (Figure 4.5a), the $\mathrm{T}_{m}$ was estimated for a range of concentrations (Figure 4.5b). By assuming non-self-complementary association of dimers, equation 2.1 can be rewritten as

$$
K_{d}=\frac{\left(\frac{C_{T}}{2}\right)\left(1-\frac{1}{2}\right)^{2}}{\frac{1}{2}}=\frac{C_{T}}{4}
$$

where $C_{T}$ is the total polypeptide concentration. For any process in equilibrium the equations $\Delta G^{\circ}=-R T \ln \left(\frac{1}{K_{d}}\right)$ and $\Delta G^{\circ}=\Delta H^{\circ}-T \Delta S^{\circ}$ are valid. By rearrangement

$$
-R T \ln \left(\frac{1}{K_{d}}\right)=\Delta H^{\circ}-T \Delta S^{\circ}
$$

Combining equation 4.1 and 4.2 and dividing this with $T_{m} \Delta H^{\circ}$ allow $\mathrm{T}_{\mathrm{m}}$ to be related to $\mathrm{C}_{\mathrm{T}}$ as

$$
\frac{1}{T_{m}}=\frac{R}{\Delta H^{\circ}} \ln \left(C_{T}\right)+\frac{\Delta S^{\circ}-R \ln 4}{\Delta H^{\circ}}
$$

By plotting $\frac{1}{T_{m}}$ versus $\ln C_{T}$ a linear relationship could be obtained for the measured $\mathrm{T}_{\mathrm{m}}$ values (Figure 4.5c). By using equation (4.1) and the linearly extrapolated equation, the $\mathrm{K}_{\mathrm{d}}$ can be estimated at any temperature of interest by

$$
\begin{gathered}
\frac{1}{T}=a \ln \left(4 K_{d}\right)+b \Leftrightarrow \\
\Leftrightarrow \ln \left(K_{d}\right)=\frac{\frac{1}{T}-b}{a}-\ln 4 \Leftrightarrow \\
\Leftrightarrow K_{d}=\frac{\mathrm{e}^{\frac{\frac{1}{T}-b}{a}}}{4}
\end{gathered}
$$


where $a$ is $\frac{R}{\Delta H^{\circ}}$ (the slope of the extrapolated curve) and $b$ is $\frac{\Delta S^{\circ}-R \ln 4}{\Delta H^{\circ}}$ (the Y-intercept of the extrapolated curve) (Figure 4.5d). Using this method on all four heterodimers, the different $K_{d}$ values could be estimated (Table 4.1).

(a)



(c)



(b)

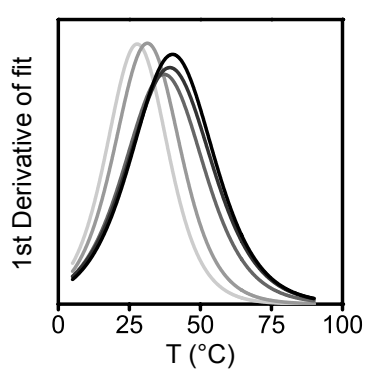

(d)



Figure 4.5: The $K_{d}$ was estimated for all combinations of heterodimers, exemplified here with EV/KV. a) Thermal denaturation curves for EV/KV at different concentrations. b) 1st derivative of each thermal denaturation curve for $\mathrm{EV} / \mathrm{KV}$ to visualize and to estimate the melting temperature at each concentration. c) The linear relationship between $\frac{1}{T_{m}}$ and $\ln C_{T}$ for $\mathrm{EV} / \mathrm{KV}$, used to estimate the $\mathrm{K}_{\mathrm{d}}$. d) Relationship between $\mathrm{K}_{\mathrm{d}}$ and temperature for $\mathrm{EV} / \mathrm{KV}$.

Due to the large difference in affinities the EKIV-polypeptide system will self-sort, forming only two out of four possible heterodimers when all polypeptides are present. However, as shown in paper I, it is not the two heterodimers with the highest affinities that are formed. Instead it is the heterodimers with the highest $(\mathrm{EI} / \mathrm{KI})$ and the lowest $(\mathrm{EV} / \mathrm{KV})$ affinities that are formed. The reason for this is depicted in Figure 4.6. When all polypeptides are mixed, they strive to form the most thermodynamically stable heterodimer as possible. EI and $\mathrm{KI}$ will predominantly form $\mathrm{EI} / \mathrm{KI}$ as it is the most thermodynamically favored heterodimer. When this heterodimer is formed, EI and KI polypeptides will be consumed in the system. The consumption will limit the possibility for the system to form both $\mathrm{EI} / \mathrm{KV}$ and $\mathrm{EV} / \mathrm{KI}$. As a result, $\mathrm{EV}$ and $\mathrm{KV}$ cannot form their most thermodynamically favorable heterodimer EV/KI or $\mathrm{EI} / \mathrm{KV}$. To lower the total energy in the system, EV and KV will heterodimerize 
into $\mathrm{EV} / \mathrm{KV}$. Furthermore, it is possible to stepwise go from one heterodimer to another by sequential addition of polypeptides. For example, addition of EI to $\mathrm{EV} / \mathrm{KV}$ will result in $(\mathrm{EV} / \mathrm{KV}+\mathrm{EI}) \rightarrow(\mathrm{EI} / \mathrm{KV}+\mathrm{EV})$. The change in heterodimers happens spontaneously as $\mathrm{K}_{\mathrm{d}}(\mathrm{EV} / \mathrm{KV})>\mathrm{K}_{\mathrm{d}}(\mathrm{EI} / \mathrm{KV})$. In the same manner, sequential addition of $\mathrm{KI}$ results in $(\mathrm{EI} / \mathrm{KV}+\mathrm{EV}+\mathrm{EI})$ $\rightarrow(\mathrm{EI} / \mathrm{KI}+\mathrm{EV} / \mathrm{KV})$. In paper I this phenomenon is shown using a fluorescent probe, whereas in paper II this is shown using dynamic light scattering experiments (DLS) ${ }^{\mathrm{b}}$.



Figure 4.6: Schematic representation of the self-sorting in the EKIV-polypeptide system. $\mathrm{EI}$ and $\mathrm{KI}$ will mainly heterodimerize into $\mathrm{EI} / \mathrm{KI}$ as this is the most stable heterodimer. As $\mathrm{EI}$ and $\mathrm{KI}$ are consumed in the system, EI/KV and EV/KI will be unable to form. EV and $\mathrm{KV}$ will thus heterodimerize into $\mathrm{EV} / \mathrm{KV}$, even though this is less stable than both $\mathrm{EI} / \mathrm{KV}$ and $\mathrm{EV} / \mathrm{KI}$.

All in all, the EKIV-polypeptide system allows formation of four different heterodimers with distinct and different affinities for dimerization, which in addition is prone to self-sorting. In paper II the polypeptides were modified with an end-terminal Cys residue to provide a reactive moiety for conjugation to maleimide-containing poly(ethylene glycol). The conjugation process is discussed in subsection 5.2.1 and the use of the resulting polypeptide-hybrids is discussed in section 6.2.

\subsection{JR-polypeptides}

The JR-polypeptides are 42 amino acid residues helix-loop-helix polypeptides and were used in paper III, IV and V. The JR-polypeptides were originally designed by Johan Rydberg et $\mathrm{al}^{91}$ and are designed to dimerize into

${ }^{\mathrm{b}}$ See subsection 7.1 .3 
anti-parallel four-helix bundles. The main JR-polypeptide that has been used in this thesis is JR2E. The amino acid sequence of JR2E can be found in Table 4.3 and corresponding helical wheel diagram when folded into an anti-parallel four-helix bundle can be seen in Figure 4.7. The design principle is roughly the same as for coiled coil design in general. The $a$ and $d$ positions constitute the hydrophobic core and are occupied mostly by hydrophobic residues such as Ala, Ile and Leu. The amino acid residues at $e$ and $b$ positions will be the interface between the dimers and are mostly occupied with Glu residues. $c, f$ and $g$ positions are mostly occupied by either hydrophilic residues or by Ala.
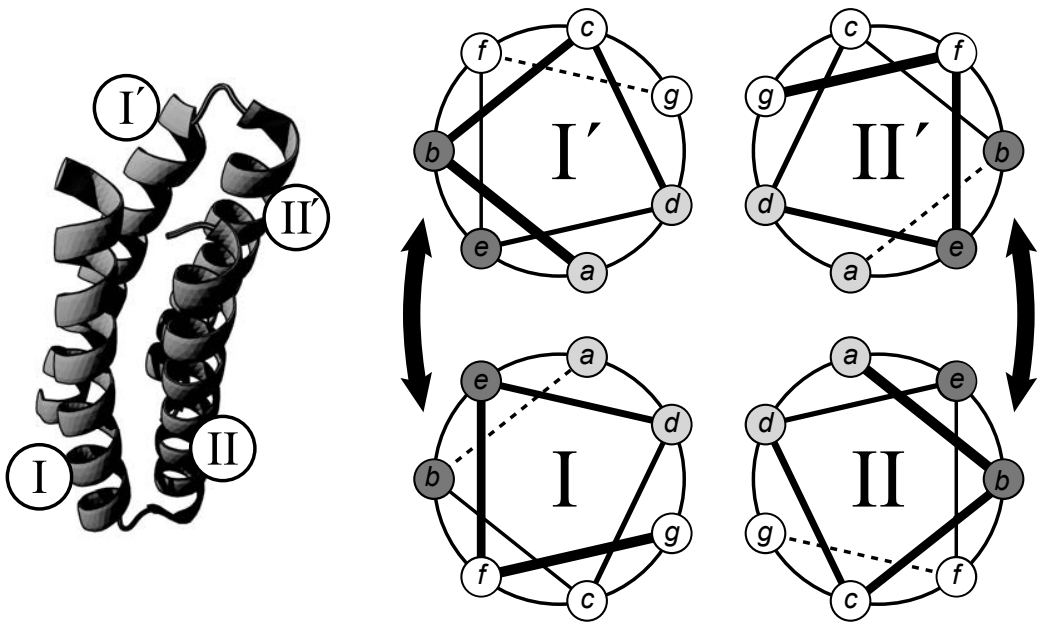

Figure 4.7: Cartoon representation of two JR-polypeptides dimerized into a four-helix bundle with corresponding helical wheel diagram.

\begin{tabular}{|c|c|c|c|c|c|c|c|c|c|c|c|c|c|c|c|c|c|c|}
\hline \multicolumn{19}{|c|}{ Sequence and heptad register } \\
\hline 1 & & & & & & & & & & & & & & & & & & 19 \\
\hline$g$ & $a$ & $b$ & $c$ & $d$ & $e$ & $f$ & $g$ & $a$ & $b$ & $c$ & $d$ & $e$ & $f$ & $g$ & $a$ & $b$ & $c$ & $d$ \\
\hline $\mathrm{N}$ & A & A & $\mathrm{D}$ & $\mathrm{L}$ & $\mathrm{E}$ & $\mathrm{K}$ & A & I & $\mathrm{E}$ & A & $\mathrm{L}$ & $\mathrm{E}$ & $\mathrm{K}$ & $\mathrm{H}$ & $\mathrm{L}$ & $\mathrm{E}$ & A & $\mathrm{K}$ \\
\hline & & & & & & & & & & & & & & & 20 & & & 23 \\
\hline & & & & & & & & & & & & & & & G & $\mathrm{P}$ & $\mathrm{V}$ & $\mathrm{D}$ \\
\hline 42 & & & & & & & & & & & & & & & & & & 24 \\
\hline$e$ & $d$ & $c$ & $b$ & $a$ & $g$ & $f$ & $e$ & $d$ & $c$ & $b$ & $a$ & $g$ & $f$ & $e$ & $d$ & $c$ & $b$ & $a$ \\
\hline G & A & $\mathrm{R}$ & $\mathrm{E}$ & $\mathrm{F}$ & A & $\mathrm{E}$ & $\mathrm{F}$ & A & Q & $\mathrm{E}$ & $\mathrm{L}$ & $\mathrm{Q}$ & $\mathrm{K}$ & $\mathrm{E}$ & $\mathrm{L}$ & $\mathrm{Q}$ & A & A \\
\hline
\end{tabular}

Table 4.3: The amino acid sequence for JR2E. 1-19 and 24-42 are the helices whereas $20-23$ is the unstructured loop region.

As the dimer interface is mostly occupied by Glu residues, similar rules for folding governs the JR2E polypeptide as for the EKIV-polypeptides. By protonization of the $\gamma$ - carboxyl group of Glu, stabilizing Glu-Glú interactions 
can occur. Protonization, and thus homodimerization, occurs in acidic conditions $(\mathrm{pH}<6)$ since the $\mathrm{pH}$ comes closer to the $\mathrm{pI}$ of the polypeptide $(\mathrm{pI}=$ 4.6). ${ }^{92}$ Furthermore, JR2E can also heterodimerize at neutral $\mathrm{pH}$ in the presence of the charge complementary polypeptide JR2K. ${ }^{93} \mathrm{JR} 2 \mathrm{~K}$ has a similar amino acid sequence as JR2E, however all Glu residues at the $e$ and $b$ positions are replaced with Lys. The Lys residues allow formation of stabilizing salt bridges upon heterodimerization with JR2E. The heterodimerization between JR2E and JR2K was used in paper Vto post-modify the created hydrogels. A third option to induce dimerization of JR2E is by metal ion coordination, a strategy that was used in paper III, IV and V. It has previously been shown that various di- and trivalent metal ions can induce folding of JR2E. ${ }^{94}$ The divalent metal ion $\mathrm{Zn}^{2+}$ induces the highest degree of helicity, which is the main reason for choosing this metal cation for the papers in this thesis.

By replacing the Val residue in the loop-region of JR2E to other more reactive moieties, it is possible to conjugate the polypeptide to other molecules. Replacing Val22 $\rightarrow$ Cys (JR2EC) gives a reactive thiol group that can be used for further post-modifications. For instance, by oxidizing JR2EC, as done in paper III, two JR2EC polypeptides can be linked via a disulfide bridge (JR2EC 2 ). Addition of $\mathrm{Zn}^{2+}$ to $\mathrm{JR}_{2} \mathrm{EC}_{2}$ result in the formation of macrosized assemblies of superstructures that could not be done with JR2E. In addition, in paper IV and V the replacement of Val22 $\rightarrow$ Lys(Alloc) was used as a synthesis step to conjugate the polypeptide to the polymer hyaluronic acid. More details about this specific modification is provided in subsection 5.2.2.

\subsection{Synthesis of peptides}

To polymerize amino acids into peptides, many different techniques has been used throughout history. ${ }^{95}$ Today, the most common technique for lab-scale synthesis of peptides is called solid phase peptide synthesis (SPPS). SPPS is the result of the pioneering work of Robert Bruce Merrifield in the 1960's, a work that in 1984 awarded him with the Nobel Prize in Chemistry. ${ }^{96}$

\subsubsection{Solid phase peptide synthesis}

In SPPS peptides are synthesized on an insoluble resin support. The resin allows purification of the product after each synthesis step by enabling removal of all soluble by-products by filtration and washing. This strategy to synthezise peptides allowed SPPS to early on be fully automated, thus making it practically possible to synthesize longer and more complex peptides. The main steps in SPPS are illustrated in Figure 4.8. At first the resin is loaded by attaching a N-terminal protected amino acid to the solid support via the carboxyl group. ${ }^{97}$ After loading the first amino acid, the peptide chain is synthesized in a linear fashion from the C-terminal to the N-terminal amino acid. This is done by 
repetitive cycles of removal of the $\mathrm{N}$-terminal protection group on the growing peptide and sequential attachment of a new $\mathrm{N}$-terminal protected amino acid. The attachment is done by adding a coupling reagent, such as HCTU' or TBTU ${ }^{\mathrm{d}}$, with the new N-terminal protected amino acid. The coupling reagent activates the carboxyl group on the free $\mathrm{N}$-terminal protected amino acid by creating a highly reactive $\mathrm{O}$-acylisourea. The $\mathrm{O}$-acylisourea can then react with the amino group of the growing peptide. The reaction results in a new peptide bond. After each reaction step, excess reactants and by-products are removed by extensive washing and filtration. The cycle is repeated until the desired sequence is obtained. Subsequently, the peptide is cleaved from the resin and side chain protective groups are removed. Depending on the type of resin that is used, the resulting peptide will either have a C-terminal carboxyl acid or a C-terminal amide.

Depending on which N-terminal- and side-chain protections that are used, different strategies for deprotection and cleavage from the resin are needed. In SPPS, two strategies are commonly used today; The Boc and the Fmoc approach, where the latter has been used in this thesis. The Boc approach is based on the difference in acid lability of the N-terminal protecting group Boc and corresponding side chain protective groups. Boc is removed by trifluoroacetic acid (TFA) while the side chain protective groups and the peptide-resin linkage are removed by anhydrous hydrogen fluoride (HF). Even though the Boc approach has many great advantages such as higher purity and thereby easier to synthesize longer peptide sequences, HF is extremely dangerous to handle since contact with water immediately converts HF to highly corrosive and toxic hydrofluoric acid. In addition, very specialized machines for automated processes that can handle HF are needed and HF may also degrade some peptides. The problems related with $\mathrm{HF}$ was one of the main reasons why Chang et al. in 1978 developed a new synthesis strategy using 9-fluorenylmethyloxycarbonyl (Fmoc) instead of Boc as the N-terminal protecting group. ${ }^{98}$ The Fmoc approach uses milder deprotection chemicals. By using a base, often piperidine dissolved in dimethylformamide (DMF), the Fmoc-group can readily be removed. The side chain protection groups and peptide-resin linkage are removed by an acid, commonly TFA. However, when the side chain protective groups are removed with TFA there is a risk that these will react with certain amino acid residues side chain and form unwanted by-products. To reduce the risk, scavenger molecules are added with the TFA when cleaving the peptides from the resin. The scavenger molecules will react with the removed protective groups, making them unavailable to react with the exposed side chains of the peptides. Depending on which amino acids the peptide contains, various scavengers are needed. Examples of scavengers used in this thesis are triisopropylsilane and 1,2-Ethanedithiol.

${ }^{\mathrm{c}}$ 2-(6-Chloro-1H-benzotriazole-1-yl)-1,1,3,3-tetramethylaminium hexafluorophosphate

${ }^{\mathrm{d}} \mathrm{O}$-(Benzotriazol-1-yl)-N,N,N,N'-tetramethyluronium tetrafluoroborate 


\section{Step 1}

Attach protected amino acid residue to resin

\section{Step 2}

Remove Fmoc protecting group

\section{Step 3}

Attach next protected amino acid

\section{Final step}

Remove protecting groups and detach peptide from the resin

\footnotetext{
* Side chain protected
}
<smiles>[R7]C(NC(=O)O)C(=O)OCCCC</smiles>

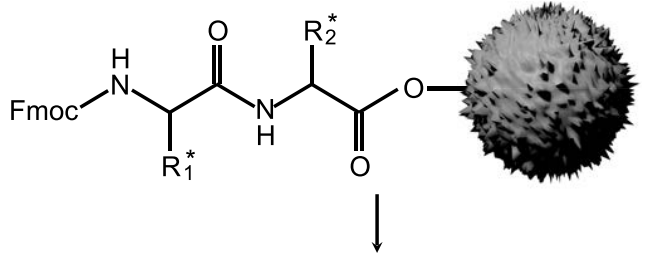

Repetitions of steps 2-3



Figure 4.8: The principle of SPPS using the Fmoc strategy. 


\subsubsection{Peptide purification}

After synthesis of a peptide, a purification step is needed to remove truncated peptides and other by-products. Peptides are typically purified by reverse phase high performance liquid chromatography (RP-HPLC), a technique that has been used in this thesis. RP-HPLC is based on the interaction of the solute with a solid stationary phase and a liquid mobile phase. In RP-HPLC a hydrophobic stationary phase is used, commonly consisting of silica particles that have been functionalized with hydrophobic alkyl chains. The mobile phase is usually an aqueous solution mixed with an organic solvent such as acetonitrile, 2-propanol or methanol. The ratio of the aqueous/organic mixture is often changed over the course of the purification, creating an eluent gradient. When injecting a sample into a column with the stationary phase filled with a solution of high water content, most molecules with any hydrophobic tendency will bind to the stationary phase. As the eluent gradient changes toward a higher content of organic solvent, more and more hydrophobic molecules tend to go into the mobile phase and elute out of the column. The eluted molecules can be detected on-line by various types of equipment attached to the RP-HPLC. Absorption spectroscopy was used in this thesis. The peptide backbone absorbs light around $190-230 \mathrm{~nm}$, which can be detected. In addition, the EKIV-polypeptides do also absorb light around $280 \mathrm{~nm}$ due to the Trp residue.

\subsubsection{Peptide identification}

The polypeptides in this thesis were all identified by Matrix-Assisted Laser/ Desorption/ Ionization Time of Flight Mass Spectrometry (MALDI-ToF MS). MALDI-ToF is a versatile method to determine the molecular weight of non-volatile macromolecules such as peptides. The sample is embedded in an UV-absorbing matrix, such as $\alpha$-cyano-4-hydroxycinnamic acid, that forms a crystalline mesh. The mesh is introduced into a high vacuum chamber and irradiated with a laser. The irradiation induces ionization of the matrix that in turn transfers protons to the sample, making it positively charged. The ionized sample can then be accelerated in an electric field and the time of flight to reach a detector is correlated to the mass-to-charge $(\mathrm{m} / \mathrm{z})$ ratio of the sample. 


\section{Chapter 5}

\section{Synthesis of polypeptide-hybrids}

Click. Boom. Amazing!

Steve Jobs

This chapter will describe how the different polypeptide-hybrids used in paper II, IV and V were synthesized. First polymers will be described in general, including the polymers used in this thesis. Furthermore, modification strategies to facilitate reactive moieties for conjugation between the polypeptides and the polymers will be described.

\subsection{Polymers}

The term polymer has already been mentioned in previous chapters of this thesis. The term polymer was coined by the Swedish chemist Jacob Berzelius in $1832,{ }^{99}$ and is derived from the Greek words poly- 'many' and -meros 'part'. A polymer is composed of several repeating residues, referred to as monomers. A polymer can either originate from a natural source, such as DNA, cellulose and chitin, or be completely synthetic such as in the case of polystyrene, polytetrafluoroethylene and polyvinylchloride. As the monomers react with each other, long polymer chains are formed. A polymer does not necessarily consist of only one type of monomer, but is often comprised of several different monomers. Depending on how the different types of monomers are distributed, various prefixes are used to describe the polymer. ${ }^{100}$ Polymers consisting of one type of monomer (-AAAAAAAA-) are called homopolymers, whereas polymers consisting of two or more monomers are called copolymers. Copolymers are in turn divided into different subcategories depending on the relative order of each monomer in the polymer chain. Alternating copolymers have an regular 
alternating pattern (-ABABABAB-), whereas in block copolymers the different monomers are divided in specific segments (-AAAABBBB-). In contrast, in statistical copolymer the monomers are randomly distributed in the polymer chain (-ABBABABB-). Furthermore, polymers consisting of one single chain is referred to as a linear polymer whereas polymers with polymeric side chains are called branched polymers. A star-shaped polymer is a specific class of branched polymer that for instance was used in paper II and V. Here several polymers are connected to a central core consisting of either a single atom or a small macromolecule. In addition, branched copolymers where the polymeric side chains are distinctly different from the main polymer chain are often categorized as grafted copolymers. The polypeptide-hybrids used in this thesis all fall into this category.

As mentioned previously, several types of both natural and synthetic polymers do exist. In the papers underlying this thesis, hyaluronic acid and poly(ethylene glycol) has been used in addition to the polypeptides.

\subsubsection{Hyaluronic acid}

Hyaluronic acid (HA), also known as hyaluronan, is a naturally occuring, non-sulfonated polysaccharide of high molecular weight $\left(10^{5}-10^{7} \mathrm{Da}\right) \cdot{ }^{101} \mathrm{HA}$ was discovered and isolated for the first time in 1934 by Karl Meyer and John Palmer by extraction from the vitreous humor of cow eyes, ${ }^{102}$ from which the polymer gets its name (Greek hyaloid 'vitreous'). The structure of HA, solved by the same research group in the 1950's, is a linear alternating copolymer consisting of the alternating saccharide units $\beta$ - 1,4 - D - glucuronic acid and $\beta$ - 1,3 - N - acetyl - D - glucosamide (Figure 5.1). The carboxyl group of the D-glucuronic acid unit makes the polymer slightly negatively charged at neutral $\mathrm{pH}$. However, under physiological conditions HA is primarily found as the sodium salt, as the salt form is more stable in comparison to the acidic form.

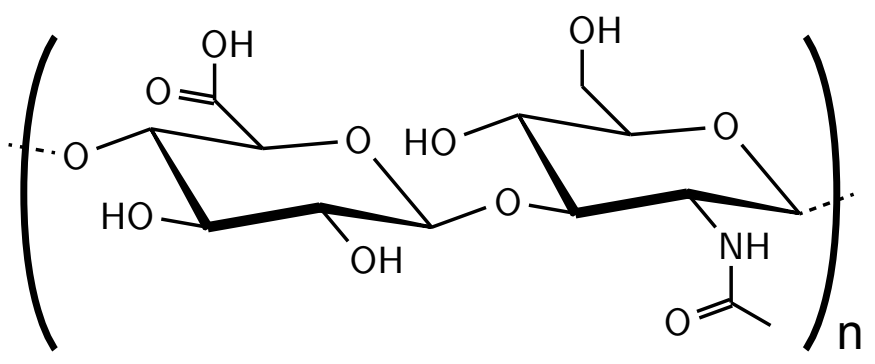

Figure 5.1: Chemical structure of hyaluronic acid.

Since its discovery, HA has been found to be one of the major constituents of the extracellular matrix (ECM) of vertebrates, for example found in the synovial 
joint fluid and in the skin and cartilage. ${ }^{101} \mathrm{HA}$ has several important biological functions in the body, such as maintaining the viscoelastic properties of liquid connective tissue and regulating the hydration of tissues. ${ }^{103,104}$ Furthermore, HA interacts with cell surface receptors (e.g. CD44, ICAM-1 and RHAMM) and HA-binding proteins to promote cell adhesion, migration and proliferation. In addition, HA can be naturally degraded by various reactive oxygen species (ROS) and enzymes (e.g. hyaluronidase ${ }^{104}$ ), and is important in the wound healing process. ${ }^{105}$ The intrinsic biological properties of HA has rendered it highly useful in many biomaterial applications over the years. HA is today clinically and commercially used in applications such as dermal fillers, wound repair, and skin care products. Furthermore, by modifying the chemical structure of HA with new functional groups, for example as done in paper IV and $\mathrm{V}$, it is possible form e.g. polymeric nanoparticles or hydrogels, using either covalent or supramolecular bonds. ${ }^{106-114}$

\subsubsection{Poly(ethylene glycol)}

Poly(ethylene glycol) (PEG) is a synthetic and hydrophilic homopolymer that is commercially available in a wide range of molecular weights $\left(\approx 10^{2}-10^{7} \mathrm{Da}\right)$. The primary structure of PEG consists of a linear polyether structure with terminating hydroxyl groups (Figure 5.2). The terminating groups are often converted into other reactive moieties to enable conjugation to other types of molecules. In addition, PEG polymers can be connected in different structural arrangements, such as star-shaped polymers.

Although some people do show hypersensitivity towards PEG, the polymer is considered to be bioinert and non-toxic ${ }^{115}$ These properties and its hydrophilicity makes PEG useful in many biomaterial applications. In drug delivery applications, low molecular weight PEG-structures are often conjugated to hydrophobic drugs and proteins (called pegylation) to improve their solubility in aqueous media. Pegylation can also improve the pharmokinetics of drugs e.g. by prolonging the retention time in the blood or by reducing the renal excretion of the drug. ${ }^{116}$ Furthermore, due to the bioinertness, PEG is often used to modify various surfaces to gain anti-fouling properties by preventing e.g. protein-binding. ${ }^{117}$ In addition, due to the well-defined structure and hydrophilicity of PEG it is widely used in hydrogel formulations. ${ }^{14,118-122}$

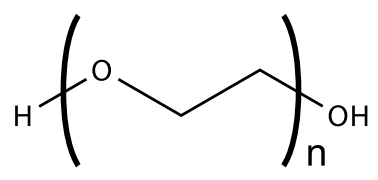

Figure 5.2: Chemical structure of poly(ethylene glycol) 


\subsection{Modification of polymers and conjugation with peptides}

Chemical modifications of polymers allow new polymers with unique properties to be created. By chemical modification, polymers can be crosslinked to form large polymeric networks, ${ }^{14,106,123,124}$ or conjugated with other functional molecules that e.g. provide self-assembling properties, ${ }^{75,110,111}$ or increase their bioactivity. ${ }^{14,124-126}$ Various strategies to chemically modify polymers exist, but a common denominator in the work done in paper II , IV and V has been to exploit click chemistry. ${ }^{127}$ Click chemistry refers to reactions that are rapid, simple to use, highly selective and give high yield. Premodification of polymers with such "click chemistry"-groups allows polymeric materials that are easy to crosslink or that can be conjugated with other functional molecules. In paper II, maleimide-thiol Michael addition was used whereas in paper IV and V strain promoted alkyne-azide 1,3-dipolar cycloaddition was used.

\subsubsection{Maleimide-thiol Michael addition}

The Michael addition reaction between maleimides and thiols is widely used to covalently bound two or more molecules. The two carbonyls in the maleimide ring structure combined with the release of ring-strain are the main driving forces for the reaction. ${ }^{128}$ The reaction is initiated by a nucleophilic attack by the thiolate anion at the $\pi$-bond of the maleimide, resulting in a strongly basic enolate intermediate (Figure 5.3). The enolate will in turn deprotonate another thiol into a thiolate anion, which in turn can continue the catalytic cycle. The protonation of the enolate results in the final addition product. Although this reaction is highly efficient and selective, some minor drawbacks exist. At $\mathrm{pH}$ above 8 , maleimides will not only begin to hydrolyze into maleic acid, but also react more readily with amines over thiols. ${ }^{129}$ Furthermore, as the thiol-containing compound must be in its reduced state to react with a maleimide, it may be necessary to pretreat it with reducing agents to cleave any disulfide bridges.

In paper II the maleimide-thiol reaction was used to conjugate thiol-terminated polypeptides to PEG. A commercially available star-shaped PEG-polymer with terminating maleimides was used as the main polymeric backbone ( $\mathrm{pMal}_{4}$, Figure 5.4). After reducing the thiol of the Cys with the reducing agent dithiothreitol, the polypeptides were added to the $\mathrm{pMal}_{4}$. Samples were reacted overnight and purified by dialysis to obtain the polypeptide-polymer conjugate. The formation of the final product was confirmed by comparing the ${ }^{1} \mathrm{H}-\mathrm{NMR}^{\mathrm{a}}$ spectra before and after polypeptide conjugation. The maleimide moiety has a distinct peak in a ${ }^{1} \mathrm{H}-\mathrm{NMR}$ spectrum $\left(300 \mathrm{MHz}, \mathrm{D}_{2} \mathrm{O}, \delta\right): 6.86(\mathrm{~s}, 2 \mathrm{H}, \mathrm{C}-\mathrm{H})$ that completely disappears after polypeptide conjugation (Figure 5.5). In addition, multiple new peaks originating from the polypeptides will appear after

\footnotetext{
a See subsection 7.1.5
} 


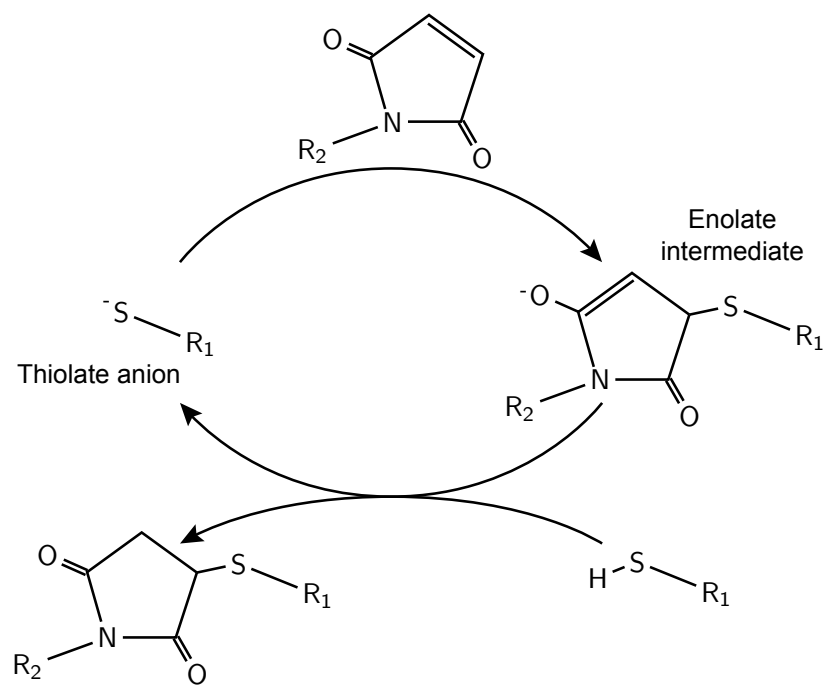

Figure 5.3: Schematics of maleimide-thiol conjugation through a Michael addition reaction. Adapted from Northrop et al. ${ }^{128}$

conjugation. As an example, since a Trp amino acid residue is present in all the EKIV-system polypeptides, ${ }^{1} \mathrm{H}$-NMR peaks originating from the aromatic indole ring will appear in the region $\left(300 \mathrm{MHz}, \mathrm{D}_{2} \mathrm{O}, \delta\right) \approx 7-8 \mathrm{ppm}$.

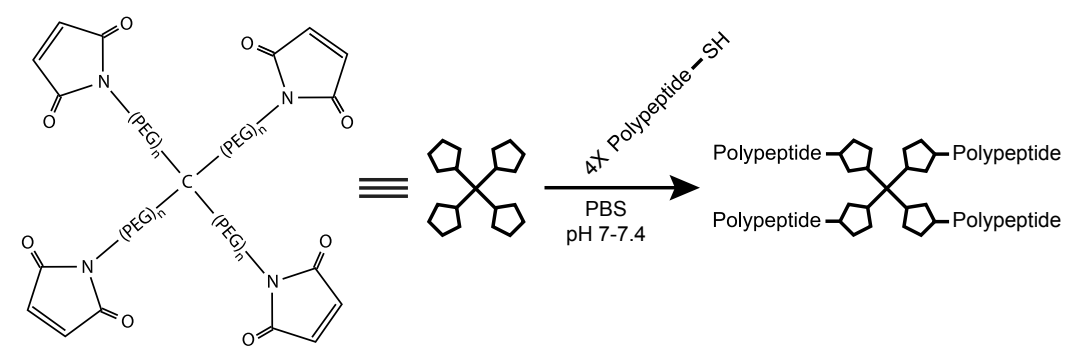

Figure 5.4: Simplified reaction scheme of the conjugation of thiol-terminated polypeptides to $\mathrm{pMal}_{4}$.

\subsubsection{Strain-promoted alkyne-azide 1,3-dipolar cycloaddition}

The reaction between alkyne-azide moieties is characterized by high selectivity and is thermodynamically highly favorable. ${ }^{130}$ The 1,3-dipolaric azide group reacts with the dipolarophilic alkyne group via a cycloaddition reaction, yielding a stable 1,2,3-triazole. In this thesis, strain-promoted alkyne-azide 

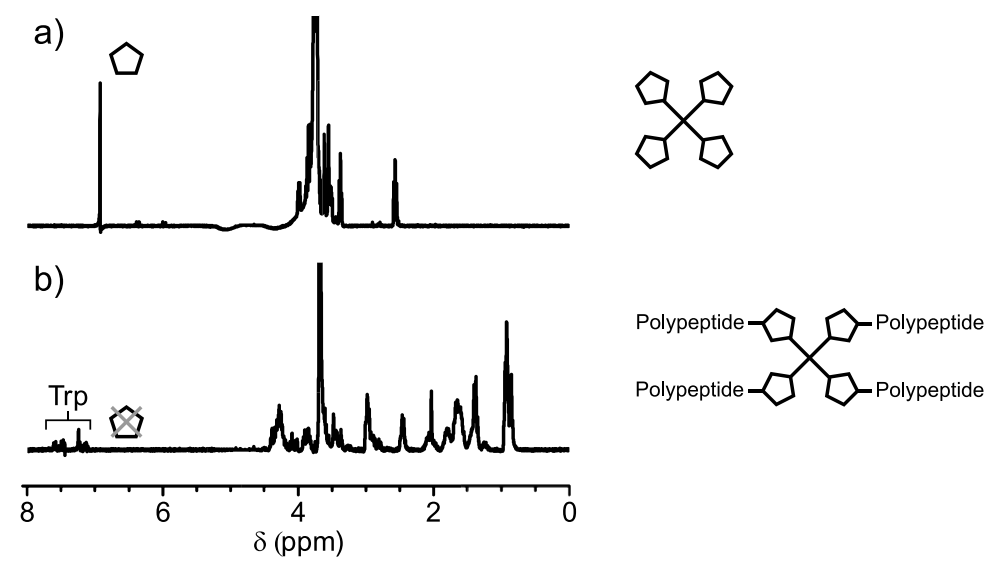

Figure 5.5: ${ }^{1} \mathrm{H}$ NMR (300 MHz) spectra of (a) $\mathrm{pMal}_{4}$ and (b) $\mathrm{pKV}_{4}$.

cycloaddition (SPAAC) was used (Figure 5.6). ${ }^{131}$ The triple bond in cyclic alkynes are energetically unfavorable and the release of the geometrical strain in the ring structure is the main driving force for the SPAAC reaction. In addition, the SPAAC reaction has many great advantages in comparison to the more traditional copper-catalyzed azide-alkyne cycloaddition. Here copper(I) ions are needed as a reagent to initiate the reaction, which is unsuitable to use in biological applications due to the toxicity of copper(I) ions. In contrast, the SPAAC reaction does not need any additional reagents to be initiated, making it more suitable for bioconjugation. Furthermore, SPAAC is considered to be bio-orthogonal as the reaction can take place in vivo. ${ }^{132}$

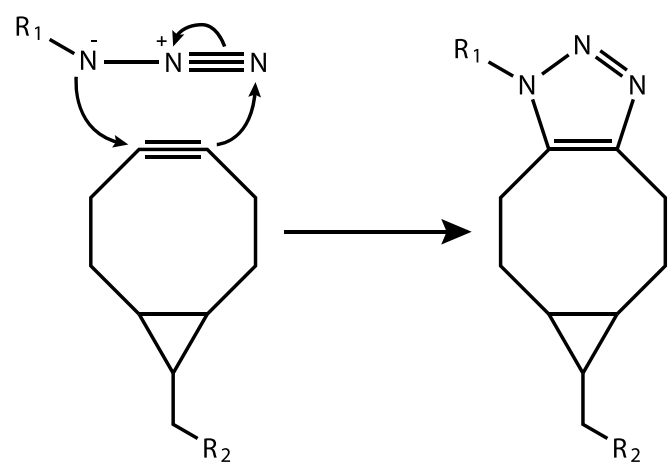

Figure 5.6: Reaction scheme for the strain-promoted alkyne-azide 1,3-dipolar cycloaddition reaction. 
In paper IV and V HA was modified with the cyclooctyne bicyclo[6.1.0]nonyne (BCN). In comparison to many other types of cyclooctynes used for SPAAC, $\mathrm{BCN}$ has a relatively high reactivity in combination with high water solubility, making it ideal for reagent-free bioconjugation under in vivo like conditions. ${ }^{133}$ To conjugate $\mathrm{BCN}$ to $\mathrm{HA}$, a commercially available $\mathrm{BCN}$ with a small linker that terminates with a primary amine group was used (Figure 5.7). Since HA has an available carboxyl group on the D-glucuronic acid unit, conjugation could be achieved with carbodiimide chemistry, resulting in a stable peptide bond and the final product (HA-BCN).

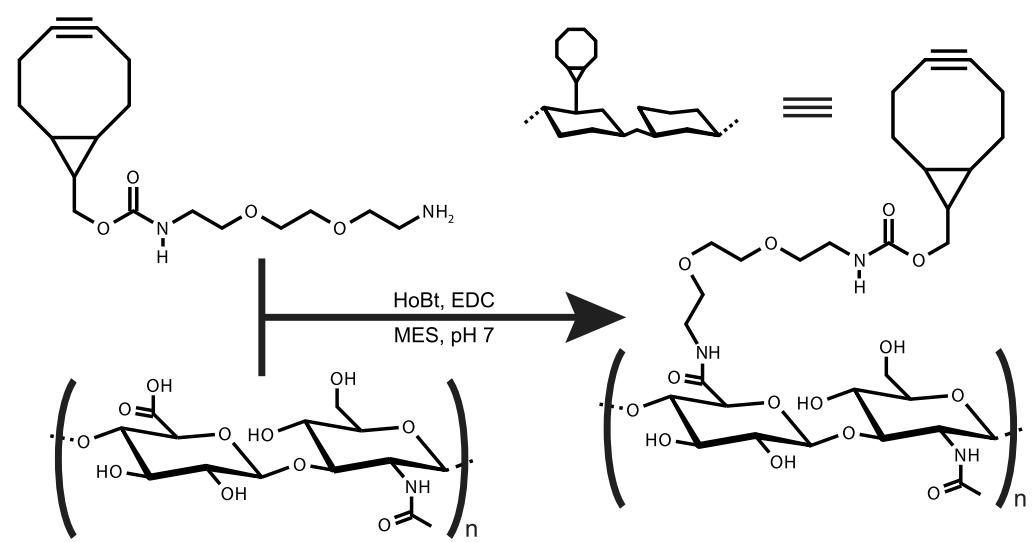

Figure 5.7: $\mathrm{BCN}$-modification of hyaluronic acid was achieved with carbodiimide chemistry.

To enable conjugation of polypeptides to HA-BCN via SPAAC, the polypeptide needs to have an azide moiety. Using an orthogonal deprotection strategy it was possible to modify the JR2E-polypeptide to incorporate an azide in the loop-region. In short, JR2E was synthesized with a Lys amino acid residue at position 22 (JR2EK) that was side chain-protected with an allylcarboxycarbonyl (Alloc). Still attached to the resin and prior the last Fmoc deprotection step in SPPS, the Alloc group was orthogonally deprotected using $\mathrm{Pd}\left(\mathrm{PPh}_{3}\right)_{4}{ }^{\text {b }}$ to give Lys(Alloc) $\rightarrow$ Lys $\left(\mathrm{NH}_{2}\right)$. The deprotected Lys $\epsilon$-amine group was in turn modified with 3-azidopropionic acid using carbodiimide chemistry to give Lys $\left(\mathrm{NH}_{2}\right) \rightarrow$ Lys $\left(\mathrm{N}_{3}\right)$. After the final cleavage and purification, JR2EK- $\mathrm{N}_{3}$ was added to HA-BCN, and sequentially conjugated via a SPAAC-reaction to yield the final product (HA-JR2EK). The conjugation, including $\mathrm{BCN}$ conjugation to $\mathrm{HA}$, was monitored using ${ }^{1} \mathrm{H}-\mathrm{NMR}$ spectroscopy (Figure 5.8).

\footnotetext{
${ }^{\mathrm{b}}$ Tetrakis (triphenylphosphine) palladium(0)
} 


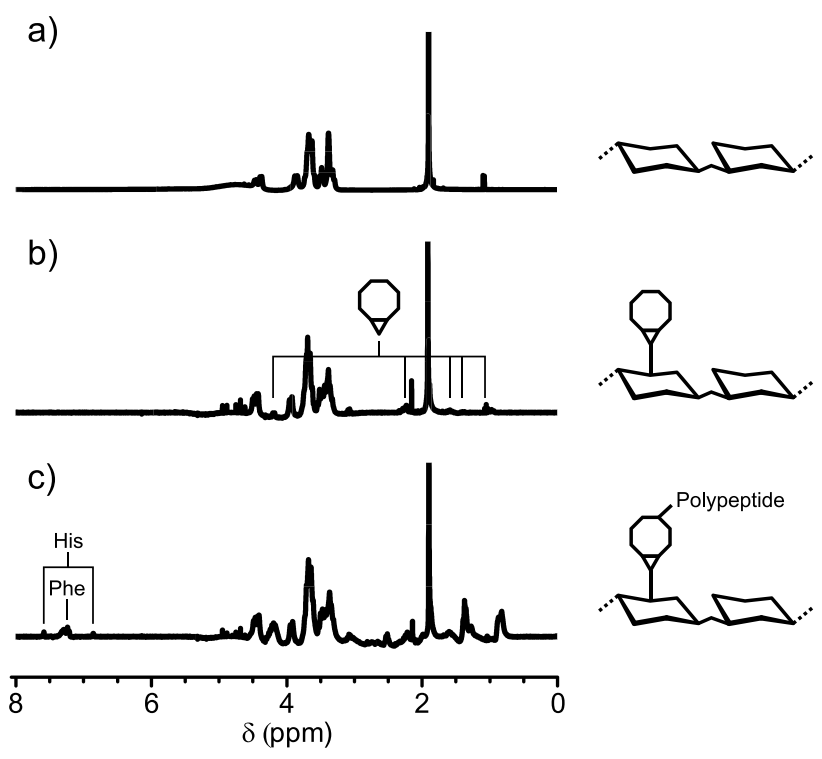

Figure 5.8: ${ }^{1} \mathrm{H}-\mathrm{NMR}(300 \mathrm{MHz})$ spectra of (a) HA (b) HA-BCN and (c) HA-JR2EK. 


\title{
Chapter 6
}

\section{Superstructures, polymeric networks and hydrogels}

\author{
[Holding spoonful of jello] 'It's a \\ solid. It's a liquid. It's a viscoelastic \\ polymer made of polypeptide chains, \\ but you can eat it-" Sam stopped, \\ realizing that she sounded like a \\ nerd. "I mean... it tastes good!"
}

Sam Sparks, from the movie

"Cloudy with a Chance of Meatballs (2009)

The focus of this chapter will be on paper II, III, IV and V. First the used terms superstructure, polymeric network and hydrogel will be defined, followed by the main results and discussions from each paper.

\subsection{Terminology}

A superstructure can be defined as "a structure upon a structure". As an example, the coiled coil motif can be regarded as a simple superstructure. As a coiled coil motif is formed, the individual polypeptides adopt an $\alpha$-helical structure. Due to the amphipathic nature, the individual $\alpha$-helices will twist around each other to form a super-helix, i.e. a superstructure. More elaborate superstructures can consist of more than two structural levels. Examples include, but are not limited to, fiber and nanotube forming coiled coil peptides, ${ }^{71,134}$ and spherically shaped nanoparticle assemblies. ${ }^{135}$ Furthermore, in paper III the terminology is used since the HLH-polypeptides first form four-helix bundles, then fibers that in turn bundles up into spheres (described more in detail in section 6.3) 
Polymeric networks can also be regarded as superstructures. As described in section 5.1, polymers consist of several linked monomeric units. The polymers can in turn form larger structures by either physically entanglement or crosslinking reactions, creating a polymeric networks. A specific type of polymeric networks that have been exploited in this thesis are hydrogels. Hydrogels are 3D polymeric networks that can store large quantities of water (> $90 \%$ of the total weight). Covalently crosslinked hydrogels are often more mechanically robust and rigid whereas supramolecularly crosslinked hydrogels are, in comparison, often softer. However, supramolecular hydrogels do often have many attractive properties such as shear thinning and self-healing. In addition to the type of crosslinking, hydrogels can be classified according to the polymeric composition. A hydrogel can be either homopolymeric, copolymeric or consist of two or more independent polymeric networks (interpenetrating polymeric network). Other common classifications of hydrogels are based on the polymers electrical charge, if the polymer origin is from natural- or synthetic sources, or simply by the physical appearance.

Due to the large water storing capability, hydrogels have been utilized in a myriad of applications, such as in agriculture, ${ }^{136}$ contact lenses, ${ }^{137}$ drug delivery devices, ${ }^{138}$ sensors, ${ }^{139}$ tissue engineering, ${ }^{140}$ wound dressings, ${ }^{141}$ cosmetic products, ${ }^{142}$ regenerative medicine ${ }^{140}$ and much more. Focusing on hydrogels in biomaterial applications, the water storing capability make hydrogels ideal for e.g. 3D cell culture. ${ }^{143-145}$ Hydrogels can be used to mimic an in vivo-like environment for cells by introducing polymers, proteins and other molecules naturally found in the ECM. This allow cells to grow and interact more naturally, as compared to cells cultured in conventional 2D layers in petri dishes.

\subsection{Polymeric networks with the EKIV-polypeptide system (Paper II)}

As described in section 4.1, the EKIV-polypeptides can be mixed-and-matched to form four different heterodimers. The resulting heterodimers have different thermal stabilities and are able to self-sort predominantly into two out of four possible combinations when all polypeptides are present in a solution. To evaluate the possibility to form hydrogels using the EKIV-polypeptides, the polypeptides were conjugated to a star-shaped PEG-polymer as described in section 5.2.1. As shown in paper II, it was possible to form hydrogels using the two combinations $\mathrm{pEI}_{4} / \mathrm{pKI}_{4}$ and $\mathrm{pEI}_{4} / \mathrm{pKV}_{4}$ at the examined concentrations (1 $2.5 \%(\mathrm{w} / \mathrm{v}))$. In contrast, neither $\mathrm{pEV}_{4} / \mathrm{pKI}_{4}$ nor $\mathrm{pEV}_{4} / \mathrm{pKV}_{4}$ were able to form hydrogels at these concentrations. The lower affinity of EV/KV $\left(\mathrm{K}_{\mathrm{d}} \approx \mu \mathrm{M}\right)$ in comparison to the other heterodimers $\left(\mathrm{K}_{\mathrm{d}} \ll \mu \mathrm{M}\right)$ was likely the main reason why $\mathrm{pEV}_{4} / \mathrm{pKV}_{4}$ did not form hydrogels at the examined concentrations. However, this cannot be the case for $\mathrm{pEV}_{4} / \mathrm{pKI}_{4}$ since the estimated $\mathrm{K}_{\mathrm{d}}$ for 


\begin{tabular}{lccc} 
Polypeptide-PEG & MRE $_{222}^{*}$ & MRE $_{222}$ & $\mathbf{T}_{m}\left({ }^{\circ} \mathbf{C}\right)$ \\
\hline $\mathrm{MEI}_{4}$ & -9.1 & 0.67 & $<20$ \\
$\mathrm{pEV}_{4}$ & -3.8 & 0.39 & $<5$ \\
$\mathrm{pKI}_{4}$ & -27.4 & 0.98 & 43 \\
$\mathrm{pKV}_{4}$ & -2.9 & 0.30 & $<5$ \\
$\mathrm{pEI}_{4} / \mathrm{pKI}_{4}$ & -34.9 & 1.00 & $>90$ \\
$\mathrm{pEV}_{4} / \mathrm{pKI}_{4}$ & -27.8 & 0.94 & 73 \\
$\mathrm{pEI}_{4} / \mathrm{pKV}_{4}$ & -33.6 & 0.99 & $>90$ \\
$\mathrm{pEV}_{4} / \mathrm{pKV}_{4}$ & -14.3 & 0.76 & 51 \\
\hline$* 10^{3} \mathrm{deg} \mathrm{cm}{ }^{2} \mathrm{dmol} \mathrm{res}^{-1}$ & & &
\end{tabular}

Table 6.1: $M \mathrm{MRE}_{222}, \frac{\mathrm{MRE}_{222}}{\mathrm{MRE}_{208}}$, and melting temperature $\left(\mathrm{T}_{m}\right)$ of individual and combined polypeptide-PEG conjugates at $\mathrm{pH} 7$ and $20^{\circ} \mathrm{C}$. The total polypeptide-PEG conjugate concentration is $0.005 \%(\mathrm{w} / \mathrm{v})$, equivalent to a total polypeptide concentration of $10 \mu \mathrm{M}$.

$\mathrm{EV} / \mathrm{KI}$ is in the same range as the $\mathrm{K}_{\mathrm{d}}$ of $\mathrm{EI} / \mathrm{KV}$. The underlying reason here is instead the fact that $\mathrm{pKI}_{4}$ tends to homoassociate. As discussed in section 4.1, this is due to the ability of KI to overcome the charge repulsion between Lys-Lys'. In addition, a positive cooperative effect was seen for all polypeptide-PEG combinations (increased $\mathrm{T}_{m}$ compared to corresponding non-PEG heterodimer). A positive cooperative effect was also seen for the $\mathrm{pKI}_{4}$, making the homoassociated $\mathrm{pKI}_{4}$ fairly stable at neutral $\mathrm{pH}$ (Table 6.1). Consequently, $\mathrm{pEV}_{4} / \mathrm{pKI}_{4}$ will not form to the same extent as $\mathrm{pEI}_{4} / \mathrm{pKV}_{4}$ at 20 ${ }^{\circ} \mathrm{C}$. However, as both a higher $\mathrm{T}_{m}$ and formation of larger assemblies was seen for the $\mathrm{pEV}_{4} / \mathrm{pKI}_{4}$ combination as compared to $\mathrm{pKI}_{4}$ alone ${ }^{\mathrm{a}}$, it could be concluded that a polymeric network was forming with $\mathrm{pEV}_{4} / \mathrm{pKI}_{4}$ as well, although not to the same extent as for $\mathrm{pEI}_{4} / \mathrm{pKV}_{4}$.

Although all combinations were not able to form hydrogels at the examined concentrations, it was possible to form larger polymeric networks with all combinations. As studied with DLS, each individual polypeptide-PEG conjugate showed an exponential decay of the autocorrelation function at shorter lag times as compared to the combinations, which all showed an exponential decay at longer lag times. Furthermore, the ability of the EKIV-polypeptides to self-sort, as discussed in section 4.1 and described in paper I, was retained when the polypeptides were conjugated to PEG. A visualization of the self-sorting phenomenon with $\mathrm{pEV}_{4} / \mathrm{pKV}_{4}$ can be seen in Figure 6.1. When $\mathrm{pEV}_{4}$ and $\mathrm{pKV}_{4}$ are mixed a polymeric network is formed, seen in DLS as a shift towards longer lag times. Addition of unconjugated $\mathrm{EI}$ to $\mathrm{pEV}_{4} / \mathrm{pKV}_{4}$ disassembles the polymeric network, seen as an exponential decay of the autocorrelation at shorter lag times. The disassembly is due to the formation of the

${ }^{\text {a}}$ Figure $5 \mathrm{G}$ in paper II 

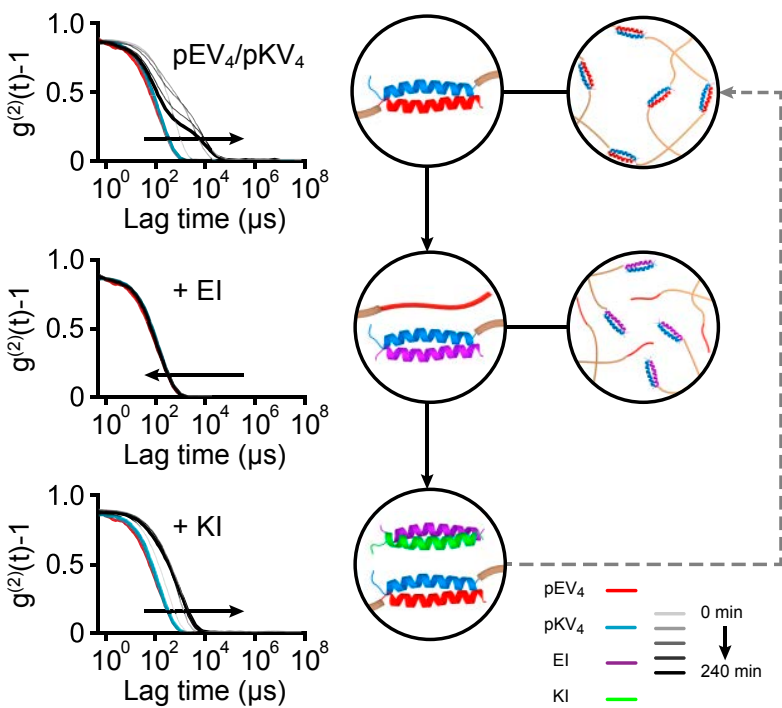

Figure 6.1: Dynamic light scattering measurements and schematic representation of the self-assembly and self-sorting phenomenon of the $\mathrm{pEV}_{4} / \mathrm{pKV}_{4}$ polymeric network. Mixing $\mathrm{pEV}_{4}$ and $\mathrm{pKV}_{4}$ will initiate the formation of a $\mathrm{pEV}_{4} / \mathrm{pKV}_{4}$ polymeric network. Subsequent addition of unconjugated EI will, due to self-sorting, disassemble $\mathrm{pEV} / 4 / \mathrm{pKV}_{4}$ $\longrightarrow \mathrm{pEV}_{4}+4 \mathrm{EI}_{4} / \mathrm{pKV}_{4}$. By adding unconjugated $\mathrm{KI}$ after this, EI/KI heterodimers will form and thus allow reassembly of $\mathrm{pEV}_{4}+\mathrm{pKV}_{4} \longrightarrow \mathrm{pEV}_{4} / \mathrm{pKV}_{4}$.

thermodynamically favored $\mathrm{EI} / \mathrm{KV}$. EI penetrates the polymeric network and forms $4 \mathrm{EI} / \mathrm{pKV}_{4}$, outcompeting $\mathrm{pEV}_{4}$ and thus disassemble the polymeric network of $\mathrm{pEV}_{4} / \mathrm{pKV}_{4}$. However, it is possible to reform the polymeric network by sequential addition of unconjugated KI. For the same reason as stated above, sequential addition of KI results in formation of the thermodynamically favored $\mathrm{EI} / \mathrm{KI}$ heterodimer. Due to this $\mathrm{pEV}_{4}$ and $\mathrm{pKV}_{4}$ can reassemble the $\mathrm{pEV}_{4} / \mathrm{pKV}_{4}$ polymeric network. Similar self-sorting as for $\mathrm{pEV}_{4}$ and $\mathrm{pKV}_{4}$ could be seen for the other polypeptide-PEG conjugates as well. The self-sorting experiments showed that it is possible to post-modulate the polymeric networks simply by adding higher affinity polypeptides.

As discussed, $\mathrm{pEI}_{4} / \mathrm{pKI}_{4}$ and $\mathrm{pEI}_{4} / \mathrm{pKV}_{4}$ were able to form hydrogels at $1-2.5 \%$ $(\mathrm{w} / \mathrm{v})$. In addition, due to the different heterodimers used, the hydrogels had different rheological properties. The $\mathrm{pEI}_{4} / \mathrm{pKI}_{4}$ hydrogel was more rigid as compared to the $\mathrm{pEI}_{4} / \mathrm{pKV}_{4}$ hydrogel, visualized by tube inversion table-top rheology in Figure 6.2a. Oscillatory rheology ${ }^{\mathrm{b}}$ measurements confirmed this observation. The $\mathrm{pEI}_{4} / \mathrm{pKI}_{4}$ hydrogel had a storage modulus (G') of $\approx 1 \mathrm{kPa}$ whereas the $\mathrm{pEI}_{4} / \mathrm{pKV}_{4}$ hydrogel had a $\mathrm{G}^{\prime}$ of $\approx 0.2 \mathrm{kPa}$ within the frequency span $0.1-100 \mathrm{rad} / \mathrm{s}$ and at $2.5 \%$ (w/v) (Figure 6.2b). The G' of the hydrogels was

\footnotetext{
${ }^{\mathrm{b}}$ See subsection 7.3 .2
} 


\subsection{POLYMERIC NETWORKS WITH THE EKIV-POLYPEPTIDE SYSTEM (PAPER II)}

thus in a biological relevant range as soft tissues. ${ }^{146}$ Furthermore, the hydrogels expressed many of the characteristics commonly associated with supramolecular hydrogels. ${ }^{147}$ The hydrogels formed rapidly upon mixing the two polypeptide-PEG conjugates since more than $50 \%$ of final G' was reached within $15 \mathrm{~s}$. The hydrogels did also show shear-thinning properties with rapid self-healing upon rupturing the hydrogel with excess oscillatory strain. The results from the rheological measurements showed that it is possible to tune and control the rheological properties of hydrogels using $\alpha$-helical polypeptides with different affinities for heterodimerization.

In ongoing studies, we have started to evaluate this hydrogel-system for 3D cell culture of hepatocytes. Hepatocyte-based cell culture models are of great interest during pharmaceutical development since many drugs are metabolized in the liver. ${ }^{148}$ The $2 \mathrm{D}$ cell culture models used today are far from accurate enough to predict this metabolism. One of the main reasons is thta $2 \mathrm{D}$ substrate often alter the cytoskeleton of the hepatocytes, and other cells, resulting in a flattened morphology. The flattened morphology limit the number of cell-cell interactions, which is necessary for normal hepatocyte function. Reduced polarization, reduced bile canaliculi formation and loss of important signaling pathways are some of the reported drawbacks of culturing hepatocytes in 2D as compared to hepatocyte function in vivo. ${ }^{149-151}$ By culturing hepatocytes in a 3D environment, hepatocytes will be exposed to a more in vivo-like environment and should provide a better in vitro model for drug screening as compared to $2 \mathrm{D}$ in vitro models.

In initial experiments, the hepatoma cell line HepG2 has been cultured in $\mathrm{pEI}_{4} / \mathrm{pKI}_{4}$ hydrogels, assembled at a total polypeptide-PEG conjugate concentration of $2.5 \%(\mathrm{w} / \mathrm{v})$ in PBS. Since the polypeptide-PEG conjugates lack cell adhesion recognition sites and due to the bioinert nature of PEG, laminin was added upon gelation. Laminin is a protein found in the ECM that promotes cell adhesion, immigration and growth of many cell types including hepatocytes. ${ }^{152,153}$ Laminin, $\mathrm{pEI}_{4}$ and $\mathrm{pKI}_{4}$ were mixed and allowed to self-assemble for five minutes prior addition of additional cell culturing medium. After $30 \mathrm{~min}, \mathrm{HepG} 2$ cells were injected into the hydrogel and sequentially cultured $\left(37^{\circ} \mathrm{C}, 5 \% \mathrm{CO}_{2}\right)$. Cell viability was examined with a Live/Dead ${ }^{\circledR}$ Viability/Cytotoxicity assay ${ }^{\mathrm{c}}$ after 1,4 and 7 days of culture using confocal laser scanning microscopy. A clear difference could be seen between the $2 \mathrm{D}$ control (no $\mathrm{pEI}_{4} / \mathrm{pKI}_{4}$, petri dish coated with laminin) and the $3 \mathrm{D}$ cell culture with $\mathrm{pEI}_{4} / \mathrm{pKI}_{4}$. In contrast to the flattened morphology seen in the $2 \mathrm{D}$ cell culture, the HepG2 cells formed spherical clusters (spheroids) over time. The formation of spheroids go well in line with previous reports regarding 3D cell culture of HepG2. ${ }^{154,155}$ The spheroids increased in size over the days, indicating that the HepG2 cells were viable. The average diameter of the spheroids at day 7 was $\approx 150 \mu \mathrm{m}$. The size indicates that oxygen diffusion to the core should be is

'See subsection 7.3.1 
sufficient. It has previously been shown that spheroids with diameters $>200 \mu \mathrm{m}$ tend to be hypoxic at the core. ${ }^{156}$ Furthermore, at day 7 it seemed that the spheroids had started to merge together, allowing cell-cell interactions between previously separated spheroids. Even though only initial experiments have been carried out so far, the $\mathrm{pEI}_{4} / \mathrm{pKI}_{4}$ hydrogel shows promising results for $3 \mathrm{D}$ cell culture of HepG2.

a)

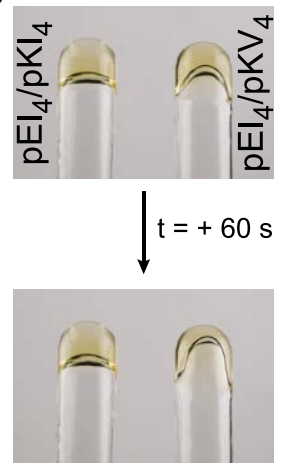

b)



Figure 6.2: a) Table-top rheology of $\mathrm{pEI}_{4} / \mathrm{pKI}_{4}$ and $\mathrm{pEI}_{4} / \mathrm{pKV}_{4}$ hydrogels at $2.5 \%(\mathrm{w} / \mathrm{v})$. b) Frequency sweep data of $\mathrm{pEI}_{4} / \mathrm{pKI}_{4}$ and $\mathrm{pEI}_{4} / \mathrm{pKV}_{4}$ hydrogels at $2.5 \%(\mathrm{w} / \mathrm{v})$.
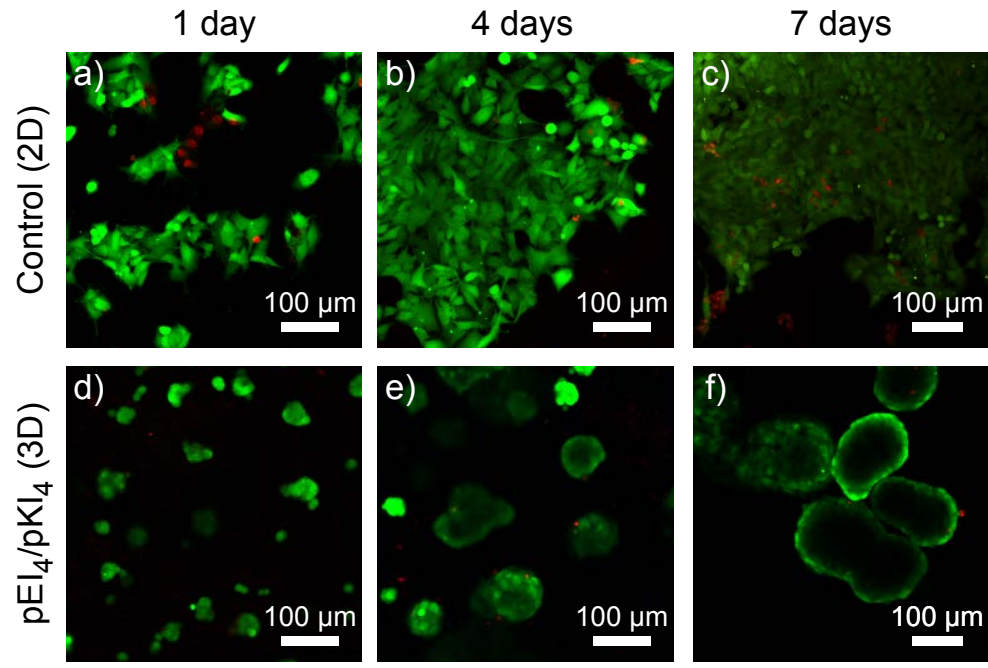

Figure 6.3: Live/Dead stained HepG2 cells and comparing the morphology in 2D and 3D cell culture. In 3D cell culture the HepG2 cells form spheroids. Live cells are stained green and dead cells are stained red. 


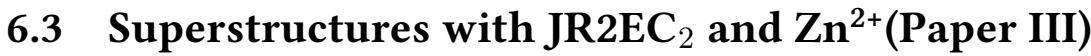

In paper III the hierarchical self-assembly process of polypeptide-based superstructures that occurs when JR2EC $\mathrm{E}_{2}$ is exposed to $\mathrm{Zn}^{2+}$ was examined. As presented in section 4.2 , JR2E is a de novo designed 42 amino acid residue polypeptide that folds into a HLH motif and can dimerize into a four-helix bundle. Modification of JR2E by replacing Val22 $\rightarrow$ Cys (JR2EC) in the loop region provides a thiol moitety that allows for further post-modifications. Examples of common thiol reactions include maleimide-thiol reactions, ${ }^{157}$ thiol-ene reactions, ${ }^{158}$ and disulfide bridge formation. Using the latter, two JR2EC polypeptides were covalently linked via a disulfide bridge by reacting the thiols in ammonium hydrogen carbonate buffer $(10 \mathrm{mM}, \mathrm{pH} 8.5)$. After removing the volatile buffer by rotary evaporation and lyophilization, $\mathrm{JR}_{2} \mathrm{EC}_{2}$ was obtained. When exposing $\mathrm{JR}_{2} \mathrm{EC}_{2}$ to $\mathrm{Zn}^{2+}$, a hierarchical self-assembly process could be initiated (Figure 6.4). The self-assembly process occurs because of the ability of $\mathrm{Zn}^{2+}$ to coordinate to amino acid residues such as Glu, Asp and His. ${ }^{159}$ Since Glu residues are the main constituent of the dimer interface when JR2E is folded (Figure 4.7), $\mathrm{Zn}^{2+}$ will induce homodimerization into four-helix bundles. Due to the disulfide bridge, several $\mathrm{JR}_{2} \mathrm{EC}_{2}$ polypeptides can homodimerize in a propagating fashion and creating a fiber. That $\mathrm{JR}_{2} \mathrm{EC}_{2}$ can form fibers when dimerizing has previously been shown in when exposing $\mathrm{JR}_{2} \mathrm{EC}_{2}$ to acidic $\mathrm{pH}$ or when adding the complementary and disulfide bridge linked polypeptide $\mathrm{JR} 2 \mathrm{KC}_{2} \cdot{ }^{72}$ However, the $\mathrm{Zn}^{2+}$-induced homodimerization of $\mathrm{JR}_{2} \mathrm{EC}_{2}$ did not result in seperate fibers, but instead resulted in large assemblies of spherical superstructures. Furthermore, as shown in paper III, an excess amount of $\mathrm{Zn}^{2+}$ was needed as compared to the total amount of Glu residues at the dimer interfaces to form stable superstructures. The hypothesis is that, in addition to the fiber-formation, lateral fiber-fiber interactions do also occur. The lateral interactions will bundle up the fibers, forming spherical superstructures. The spherical superstructures will in turn cluster together, forming larger assemblies in a diffusion limited aggregation manner. ${ }^{160}$ The formation of the large assemblies could easily be seen by the naked eye (Figure $6.4 \mathrm{~b}$ ), but could further be visualized using conventional optical light microscopy ${ }^{\mathrm{d}}$ (Figure 6.4c) and scanning electron microscopy ${ }^{\mathrm{e}}$ (Figure 6.4d).

Since the superstructures form as a result of supramolecular interactions, they could be disassembled simply by decreasing the concentration of $\mathrm{Zn}^{2+}$. As shown in paper III, the disassembly could be triggered either by $\mathrm{Zn}^{2+}$ depletion by adding preformed superstructures to a solution with no, or lower, $\mathrm{Zn}^{2+}$ concentration, or by adding the $\mathrm{Zn}^{2+}$-chelating agent EDTA ${ }^{\mathrm{f}}$. Furthermore, it was also possible to disassemble the superstructures by reducing the disulfide bridge using TCEP' ${ }^{\text {. TCEP reduces JR2EC }} 2 \longrightarrow 2 \times$ JR2EC, which will break

\footnotetext{
${ }^{\mathrm{d}}$ See subsection 7.2 .1

eSee subsection 7.2.3

${ }^{\mathrm{f}}$ Ethylenediaminetetraacetic acid

gTris(2-carboxyethyl)phosphine
} 
a)

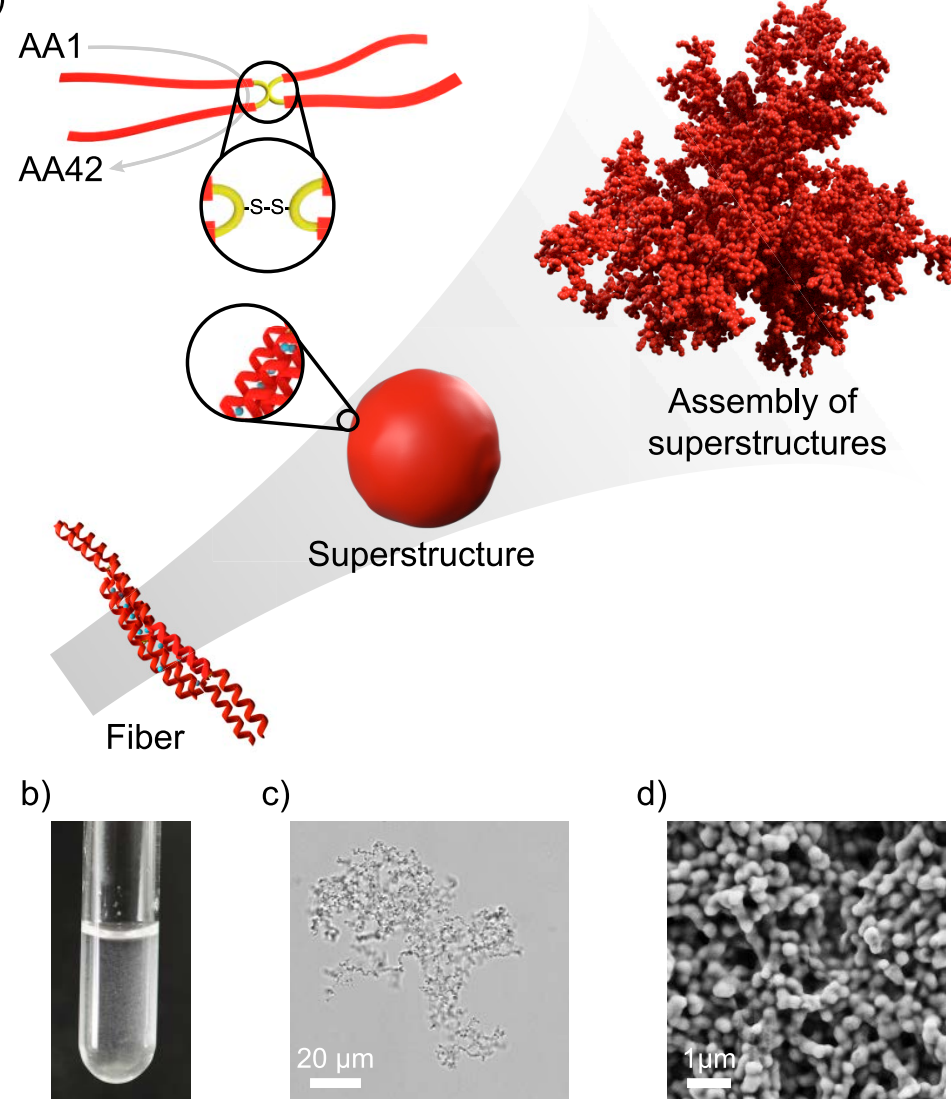

Figure 6.4: a) Schematic representation of the hierarchical self-assembly process of $\mathrm{JR}_{2 \mathrm{EC}}$ to assemblies of superstructures when exposed to $\mathrm{Zn}^{2+}$. b) Photograph of JR2EC 2 $(50 \mu \mathrm{M})$ incubated in $\mathrm{Zn}^{2+}(4 \mathrm{mM})$. c) Optical micrograph of an assembly of superstructures. d) Scanning electron micrograph of the superstructures.

down the fibers to single four-helix bundles. Regardless of which approach that was used to disassemble the superstructures, the disassembly rate could be tuned by varying the concentration of either the $\mathrm{Zn}^{2+}$ or the TCEP.

Due to the assembly and disassembly properties of the superstructures, it was possible to both encapsulate and release various compounds. In paper III this possibility was shown by encapsulating and releasing JR2EC-functionalized gold nanoparticles (JR2EC-AuNP), the fluorophore Rhodamine B, and the oligothiophene p-FTAA. ${ }^{161}$ The possibility to encapsulate AuNPs within these or similar peptide structures are of interest in biosensing applications as it allows for e.g. highly sensitive colorimetric detection of proteolytic activities due the optical properties of AuNPs. ${ }^{162,163}$ Furthermore, the possibility to encapsulate 
and release molecular compounds are of interest in e.g. drug delivery applications. Although Rhodamine B does not have any therapeutic value to date and was chosen as a model compound, p-FTAA have recently shown great therapeutic potential as it reduces the toxicity of prions and amyloid- $\beta$ peptides. ${ }^{164-166}$

The resulting pink hue of the assemblies with encapsulated JR2EC-AuNPs indicated that the JR2EC-AuNPs were well-dispersed among the superstructures (Figure 6.5a). This assumption could be made due to the optical properties of AuNPs. When the interparticle distance between AuNPs decreases, the AuNPs absorbs light at higher wavelengths. The phenomenon is called plasmonic coupling and results in a visible color change from red to blue depending on the interparticle distance. ${ }^{167}$ The well-dispersity could be further confirmed by examining the superstructures with encapsulated AuNPs with transmission electron microscopy ${ }^{\text {h }}$ (Figure 6.5b). Due to the high electron density of gold in comparison to all other atoms that constitute the superstructures, the AuNPs could easily be seen as high contrasting black dots. In addition to the possibility to encapsulate AuNPs, in paper III it is also shown that it was possible to release the encapsulated AuNPs using EDTA.

As mentioned, the possibility to encapsulate Rhodamine B and p-FTAA in the superstructures was examined. As both compounds contain carboxylate groups (Rhodamine B has one whereas p-FTAA has four) the hypothesis was that these would aid in the encapsulation. As shown in paper III both compounds could be encapsulated, although to a very different degree. Rhodamine B was encapsulated to a low degree with an encapsulation efficiency of only $0.4 \%$. In contrast, p-FTAA was encapsulated in the superstructures to a much higher degree (75\%) (Figure 6.5c-d). Furthermore, a seven day long release study was conducted with p-FTAA to investigate if it was possible to control the release rate of the encapsulated compound. As shown in paper III, it was possible to control the disassembly rate of the superstructures by $\mathrm{Zn}^{2+}$ depletion. Using the same method, preformed p-FTAA containing superstructures were added to solutions with $0-1.5 \mathrm{mM} \mathrm{Zn}^{2+}$ and the p-FTAA release was monitored for seven days. Already within the initial six hours a clear difference could be seen between the different samples (Figure 6.5e). Moreover, for e.g. superstructures


span. The results indicate that it is fully plausible to tune the release of compounds using the superstructures.

To conclude this part, $\mathrm{Zn}^{2+}$ could be used to induce JR2EC 2 to form large assemblies of superstructures that further could be used for encapsulation and release of nanoparticles and molecular compounds. However, one aspect that has not yet been discussed is potential problems using these kinds of $\mathrm{Zn}^{2+}$-containing systems in biomaterial applications. Although $\mathrm{Zn}^{2+}$ are

${ }^{\mathrm{h}}$ See subsection 7.2 .2 
a)

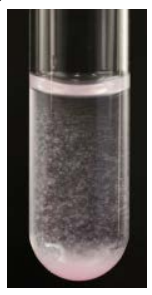

b)

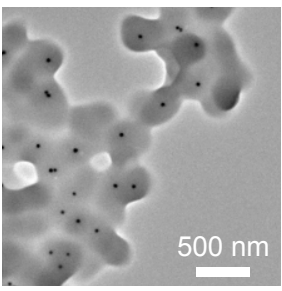

c)

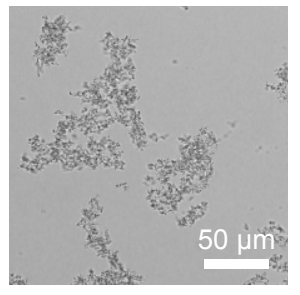

d)

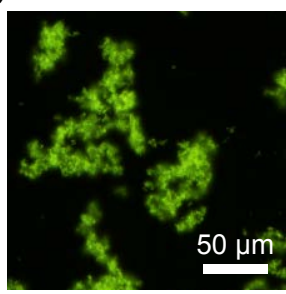

e)

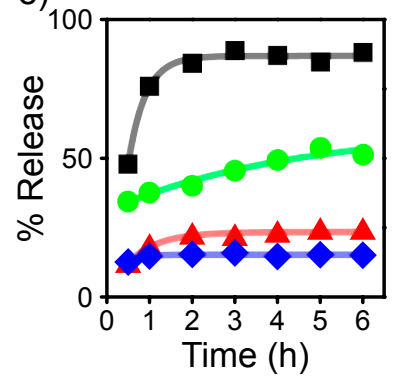

Figure 6.5: a) Photograph of JR2EC $2(50 \mu \mathrm{M})$ and JR2EC-AuNP $(\approx 0.4 \mathrm{nM})$ incubated in $\mathrm{Zn}^{2+}(4 \mathrm{mM})$. b) Transmission electron micrograph of superstructures with encapsulated JR2EC-AuNP. c) Optical micrograph and d) epi-fluorescence micrograph of superstructures with encapsulated p-FTAA. e) p-FTAA release profiles of the initial 6 hours for superstructures incubated in $0 \mathrm{mM} \mathrm{Zn}^{2+}(\boldsymbol{\square}), 0.5 \mathrm{mM} \mathrm{Zn}^{2+}(\boldsymbol{\bullet}), 1 \mathrm{mM} \mathrm{Zn}^{2+}(\mathbf{\Delta})$ and $1.5 \mathrm{mM}$ $\mathrm{Zn}^{2+}(\bullet)$

important for normal body function, ${ }^{168}$ elevated levels of free $\mathrm{Zn}^{2+}$ (i.e., $\mathrm{Zn}^{2+}$ that is not bound to proteins or other biomolecules in vivo) can potentially be cytotoxic. Work conducted by Bozym et al. shows that as little as $100 \mathrm{nM}$ of free $\mathrm{Zn}^{2+}$ may lead to declined viability for several cell types under normal cell culture conditions. ${ }^{169}$ It is thus important to consider the concentration of free $\mathrm{Zn}^{2+}$ if these superstructures should be used for e.g. drug delivery applications in vivo. However, $\mathrm{Zn}^{2+}$ do show antimicrobial properties, ${ }^{170,171}$ and have been demonstrated to promote wound healing. ${ }^{172}$ Applications where any of these features are needed, combined with the potential to release molecular compounds, can thus be potential future applications for the superstructures.

\section{4 $\mathrm{Zn}^{2+}$-responsive hydrogels with HA-JR2EK (Paper IV)}

In paper IV the $\mathrm{Zn}^{2+}$-responsive polypeptide JR2EK was used to self-assemble HA-based hydrogels. As described in subsection 5.1.1, HA has desirable intrinsic properties that makes it useful in biomaterials. In addition, $\mathrm{HA}$ and $\mathrm{Zn}^{2+}$ in 
combination are to date clinically evaluated and commercially used to reduce scare formation after surgery and to promote wound healing. ${ }^{173,174}$ As described in subsection 5.2.2, a SPAAC reaction was utilized to conjugate JR2EK to the BCN-modified HA (HA-JR2EK). A final modification degree of $\approx 4 \%$, compared to initial carboxyl groups on HA, was estimated based on the ${ }^{1} \mathrm{H}-\mathrm{NMR}$ signals originating from His and Phe amino acid residues of the polypeptide.

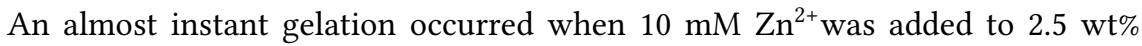
HA-JR2EK at neutral pH, resulting in a soft and sticky hydrogel (Figure 6.6a). The gelation process is caused by the homodimerization of JR2EK into four-helix bundles. The homodimerization of the polypeptides and the assembly into large polymeric networks was confirmed by CD and DLS, respectively. With increased concentration of $\mathrm{Zn}^{2+}$ an increased helicity was observed in $\mathrm{CD}$, reaching a maximum helicity at $\geq 4 \mathrm{mM} \mathrm{Zn}^{2+}$ at 0.1 wt\% HA-JR2EK concentration (Figure 6.6b). Subsequent addition of EDTA decreased the helicity to its initial value, indicating that JR2EK was unfolded. Furthermore, an increase in lag time could be seen in DLS when $\mathrm{Zn}^{2+}$ were added to $1 \mathrm{wt} \%$ HA-JR2EK, indicating formation of larger polymeric networks (Figure 6.6c). Interestingly, as seen in Figure 2c-d in paper IV, at 0.1 and $0.5 \mathrm{wt} \%$ of HA-JR2EK the opposite behavior could be seen with decreased lag times with increased $\mathrm{Zn}^{2+}$ concentration. At these lower concentrations of HA-JR2EK, intramolecular interactions seem to be more predominant compared to intermolecular interactions between separate HA-JR2EK polymer chains. At $\geq 1 \mathrm{wt} \%$ intermolecular interactions will be more predominant, resulting in formation of a hydrogel. Furthermore, the mechanical properties of the hydrogel were

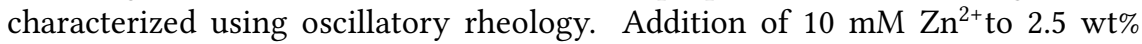
HA-JR2EK increased both the G' and G" values as compared to HA-JR2EK prior $\mathrm{Zn}^{2+}$ addition (Figure 6.6d). Furthermore, the $\mathrm{Zn}^{2+}$-induced HA-JR2EK hydrogel showed a liquid-like behavior at low frequencies with a cross point of G' and G" at $\approx 20 \mathrm{rad} / \mathrm{s}$.

In paper III the preformed superstructures could be completely disassembled by $\mathrm{Zn}^{2+}$ depletion or by degrading $\mathrm{JR}_{2} \mathrm{EC}_{2}$. Similar disassembly and degradation experiments were conducted for the hydrogel. JR2EC-AuNPs were added to HA-JR2EK prior adding $\mathrm{Zn}^{2+}$. The self-assembly resulted in hydrogels with well-distributed AuNPs, as indicated by the pink hue of the hydrogel. To examine the possibility to disassemble the hydrogels using $\mathrm{Zn}^{2+}$ depletion, hydrogels were submerged in buffers with and without additional $\mathrm{Zn}^{2+}$. The hydrogels were put on to an orbital shaker and the AuNP release caused by hydrogel disassembly was monitored using absorption spectroscopy. Hydrogels in buffers containing no additional $\mathrm{Zn}^{2+}$ lost their mechanical integrity over time and dissembled (Figure 6.7a). In contrast, when additional $\mathrm{Zn}^{2+}$ was added to the buffer little to no disassembly occurred within the same time span. Furthermore, hyaluronic acid can be enzymatically degraded by several enzymes, e.g. hyaluronidase (Hya), $\mathrm{b}^{-}{ }^{-}$-glucuronidase, and $\beta-\mathrm{N}$-acetyl-hexosaminidase. ${ }^{104}$ 
a)

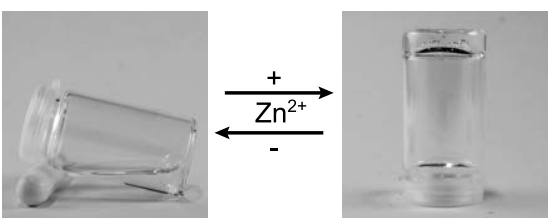

b)



c)

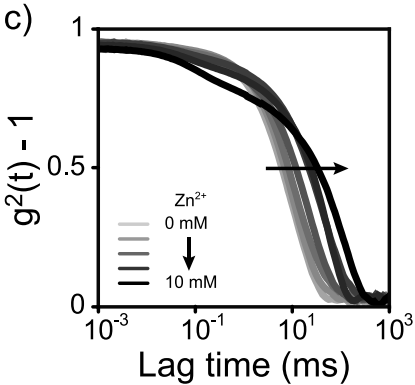



Figure 6.6: a) Table-top rheology of $2.5 \mathrm{wt} \%$ HA-JR2EK with and without $\mathrm{Zn}^{2+}$. b) CD spectra of $0.1 \mathrm{wt} \%$ HA-JR2EK with and without $10 \mathrm{mM} \mathrm{Zn}^{2+}$ and $\theta_{222}$-value related to the $\mathrm{Zn}^{2+}$ concentration (inset). c) DLS measurements of $1 \mathrm{wt} \%$ HA-JR2EK with increased concentrations of $\mathrm{Zn}^{2+}$. d) Oscillatory rheological frequency sweeps of $2.5 \mathrm{wt} \%$ HA-JR2EK with and without $10 \mathrm{mM} \mathrm{Zn}^{2+}$ at $1 \%$ strain.

By adding varied amounts of Hya to the hydrogels, it was possible to tune the degradation rate of the hydrogel (Figure 6.7b) Interestingly, a distinct difference could be seen between samples where Hya was encapsulated in the hydrogel and where Hya instead was added to the surrounding buffer. Hydrogels where Hya was encapsulated had a much quicker degradation rate as compared hydrogels where Hya was added to the buffer. This indicates that the diffusion of Hya into the hydrogel was rather limited, resulting in a more "surface erosion"-like degradation. ${ }^{175}$ In contrast, as the encapsulated Hya is more evenly distributed within the hydrogel, a more "bulk erosion"-like degradation occurred in these hydrogels. The results from the experiments showed that it is possible to tune the disassembly rate of the hydrogels using either $\mathrm{Zn}^{2+}$ depletion or enzymatic degradation.

The degradation of HA by ROS has been linked to several inflammatory-related diseases in vivo, such as rheumatoid arthritis and chronic wounds. ${ }^{176}$ However, low molecular weight HA has shown antioxidant activity, which is more pronounced when coordinated with metal ions, such as $\mathrm{Zn}^{2+} .{ }^{177,178}$ To examine whether the HA-JR2EK hydrogel showed antioxidant activity, ROS scavenging experiments were conducted using human neutrophil granulocytes. Neutrophils 
a)

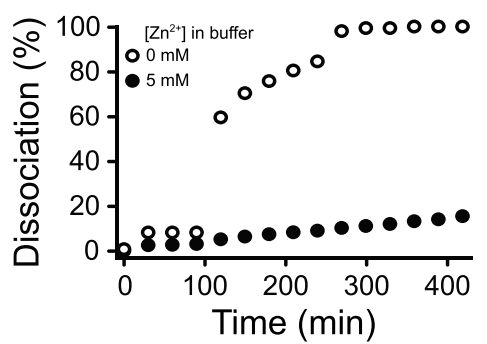

b)

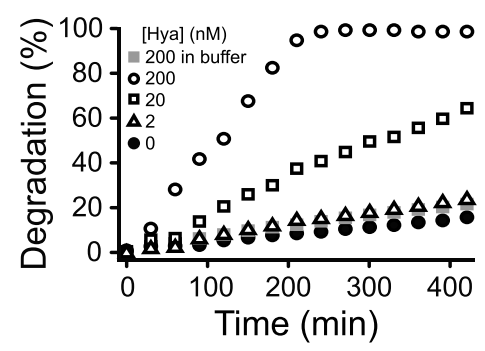

Figure 6.7: a) Dissociation over time of $2.5 \mathrm{wt} \%+10 \mathrm{mM} \mathrm{Zn}^{2+}$ hydrogels with and without additional $\mathrm{Zn}^{2+}$ added to the buffer. b) Enzymatic degradation of $2.5 \mathrm{wt} \%+10 \mathrm{mM}$ $\mathrm{Zn}^{2+}$ hydrogels with 0-200 $\mathrm{nm}$ hyaluronidase.

are one of the most ROS-contributing cells during an inflammatory response. ${ }^{179}$ Neither HA-JR2EK, $\mathrm{Zn}^{2+}$ or the combination induced a production in ROS as compared the control (Figure 6.8). However, addition of phorbol 12-myristate 13-acetate (PMA)will stimulate neutrophils to produce ROS. PMA-stimulated neutrophils in samples where $\mathrm{Zn}^{2+}$ was present showed a decrease in ROS production as compared to the control. However, HA-JR2EK alone did not show such a decrease in ROS production, suggesting that $\mathrm{Zn}^{2+}$ was the main reason for the decreased production of ROS in the experiments. Although HA-JR2EK may not be involved in the underlying mechanism for decreased ROS production, the hydrogel still is an interesting platform for scavenging ROS in biomaterial applications. For instance, the hydrogels could be used as a means to deliver $\mathrm{Zn}^{2+}$ through $\mathrm{Zn}^{2+}$-depletion. In turn, the $\mathrm{Zn}^{2+}$ would be able to reduce $\mathrm{ROS}$ and thus consequently also be able to reduce ROS-mediated degradation of HA.

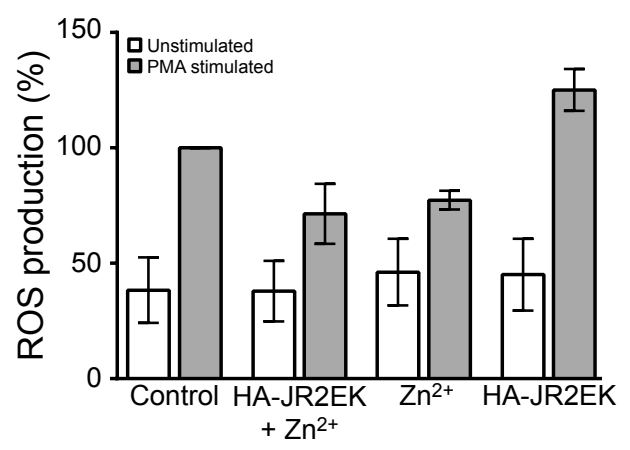

Figure 6.8: ROS production by human neutrophil granulocytes with \pm HA-JR2EK (2.5 $\mathrm{wt} \%) \pm \mathrm{Zn}^{2+}(10 \mathrm{mM})$ before and after stimulation with PMA. 


\subsection{Hydrogels with HA-JR2EK and $\mathbf{p}\left(\mathrm{N}_{3}\right)_{8}($ Paper V)}

When conjugating JR2EK to HA-BCN, roughly half of all available BCN-groups were reacted with an azide. The remaining unreacted $\mathrm{BCN}$-groups could hence be used for further SPAAC reactions, e.g. to form covalent crosslinked hydrogels as done in paper V. Addition of an 8-armed star-shaped PEG with terminating azides $\left(\mathrm{p}\left(\mathrm{N}_{3}\right)_{8}\right)$ to HA-JR2EK resulted in the formation of a fully transparent hydrogel (Figure 6.9a-b). The gelation was the result of the SPAAC reaction between the $\mathrm{BCN}$ groups on hyaluronic acid and the azide moieties on $\mathrm{p}\left(\mathrm{N}_{3}\right)_{8}$, resulting in covalent 1,2,3-triazole bonds between the two polymers. By varying the total polymer concentration, the final $G$ ' of the hydrogels could be tuned in the range of $\approx 200-500 \mathrm{~Pa}$ (Figure $6.9 \mathrm{c}$ ), which is in a biologically relevant stiffness range for mimicking of soft tissues. ${ }^{146,180}$ Furthermore, by varying the temperature the gelation kinetics could be tuned (Figure 6.9d). For instance, at $20{ }^{\circ} \mathrm{C}$ the $2.5 \%(\mathrm{w} / \mathrm{v})$ hydrogels had a gelation time (defined as $\mathrm{G}^{\prime}=\mathrm{G}^{\prime}$ or a phase angle of $45^{\circ}$ ) of $15 \mathrm{~min}$. By increasing the temperature to 37 ${ }^{\circ} \mathrm{C}$ the gelation time could be reduced to just $3 \mathrm{~min}$. The quicker gelation kinetics at a more in vivo-relevant temperature is a good feature in applications involving e.g. 3D cell encapsulation. To encapsulate well-dispersed cells in 3D, the hydrogel must form rapid enough to prevent cells from sedimenting and accumulating at the bottom of the hydrogel. ${ }^{124}$ In addition, as shown in paper $\mathrm{V}$, the hydrogel had high water storing capability $(\approx 99 \%)$ and good swelling behavior.

Although improved gelation kinetics could be achieved by increasing the temperature, the gelation time is not as rapid as many supramolecular hydrogels, such as the $\mathrm{pEI}_{4} / \mathrm{pKI}_{4}$ and $\mathrm{pEI}_{4} / \mathrm{pKV}_{4}$ hydrogels used in paper II that reached their final G' in less than $15 \mathrm{~s}$. However, as the HA is functionalized with JR2EK it was possible to crosslink the hydrogel supramolecularly simultaneously with the covalent SPAAC reaction. In paper $\mathrm{V}$ this is shown using $\mathrm{Zn}^{2+}$ and acidic $\mathrm{pH}$. $\mathrm{Zn}^{2+}$ chelation by JR2EK induce folding and dimerization of the polypeptide into four-helix bundles. In addition, at acidic $\mathrm{pH}$ the Glu residues will be protonated and able to form intermolecular hydrogen bonds between $\mathrm{Glu}-\mathrm{Glu}$ residues. Addition of $10 \mathrm{mM} \mathrm{Zn^{2+ }}$ upon mixing $2.5 \%$ $(\mathrm{w} / \mathrm{v})$ HA-JR2EK and $\mathrm{p}\left(\mathrm{N}_{3}\right)_{8}$ resulted in an instant increase in G' from 1 to 100 $\mathrm{Pa}$ (Figure 6.10a). In addition, the G' steadily increased over time, indicating that the SPAAC reaction between HA-JR2EK and $\mathrm{p}\left(\mathrm{N}_{3}\right)_{8}$ could occur even though HA-JR2EK was supramolecularly crosslinked. Furthermore, in comparison to hydrogels without $\mathrm{Zn}^{2+}$, an increased value of the phase angle could be noted (Figure 6.10b). The reason is an increased G", which means that the hydrogel is more viscous and can dissipate more energy as heat if the hydrogel is deformed. Furthermore, as shown in Figure $4 \mathrm{c}$ in paper $\mathrm{V}$ it was possible to induce homodimerization of JR2EK after forming the hydrogel as well. 
a)

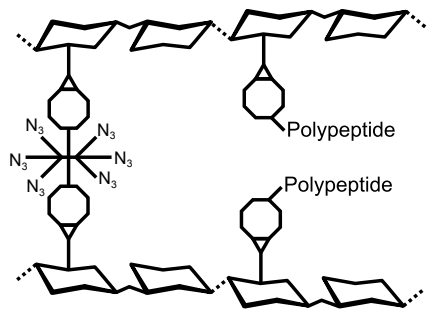

c)

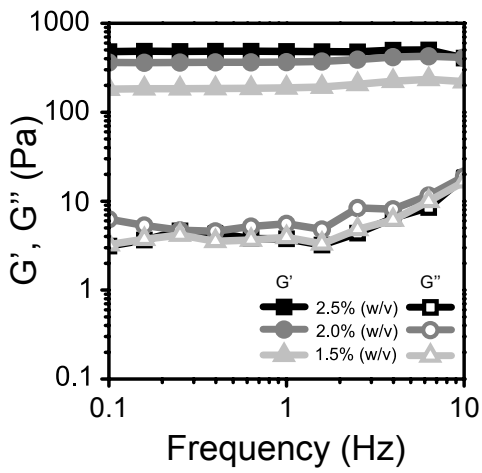

b)

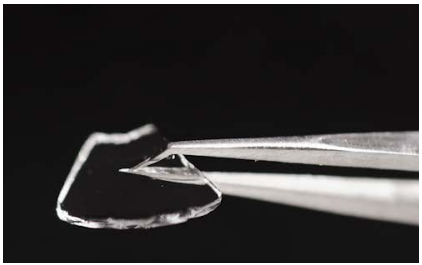

d)

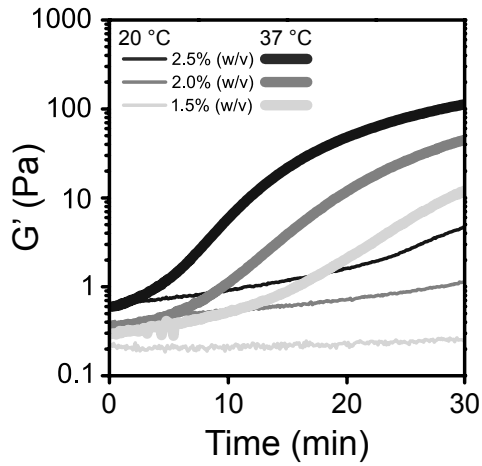

Figure 6.9: a) Schematic representation of HA-JR2EK covalently crosslinked with $\mathrm{p}\left(\mathrm{N}_{3}\right)_{8}$. b) Photograph of a $\mathrm{p}\left(\mathrm{N}_{3}\right)_{8}$-HA-JR2EK hydrogel $(2.5 \%(\mathrm{w} / \mathrm{v}))$. c) Oscillatory frequency sweeps of fully gelled $1.5-2.5 \%(\mathrm{w} / \mathrm{v})$ hydrogels. d) Gelation kinetics, showing the difference in $\mathrm{G}^{\prime}$ at 20 and $37^{\circ} \mathrm{C}$.

Instead of using JR2EK for tuning the mechanical properties of the hydrogel, the polypeptide can be used for compositional post-modifications of the hydrogel. As a proof of concept, the fluorophore $\mathrm{Cy} 5$ was conjugated to the JR2EK-complimentary polypeptide JR2KK (JR2KK-Cy5). By adding JR2KK-Cy5 to JR2EK-functionalized hydrogels, heterodimerization could be used as a means to supramolecularly load and post-modify the hydrogel with Cy5. A significant difference in uptake of JR2KK-Cy5 could be seen between the control and the JR2EK-functionalized hydrogel (Figure 6.10c). Although the control hydrogel was able to take up some of the JR2KK-Cy5 (44 $\pm 3 \%$ ), the HA-JR2EK hydrogel was able to take up much more $(91 \pm 1 \%)$. In addition, after seven days of incubation in PBS the polypeptide-functionalized hydrogel retained its distinct blue color, whereas the control was almost transparent (Figure 6.10d). These results indicate that it was possible to post-modify the HA-JR2EK hydrogel using heterodimerization. However, due to the uptake in the control, more experiments must be conducted to confirm that heterodimerization between JR2EK and JR2KK was the major reason for the greater uptake of JR2KK-Cy5 in the HA-JR2EK hydrogel. One such experiment could be to use a JR2KK-Cy5 that 
a)

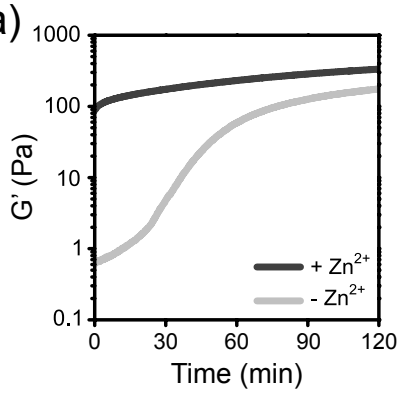

c)

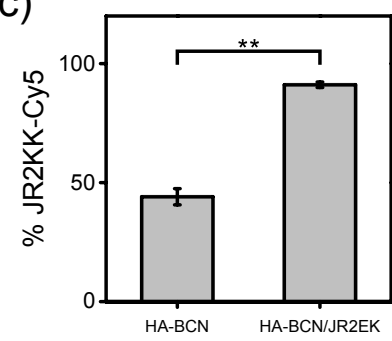

b)

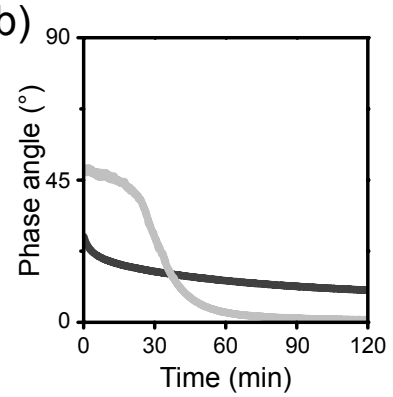

d)



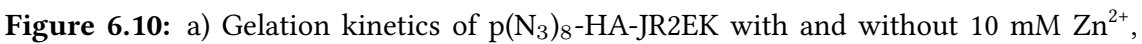
showing the difference in G' and b) phase angle. c) Loading of JR2KK-Cy5 in hydrogels functionalized and not functionalized with JR2EK $\left.\left({ }^{* *} \mathrm{P}<0.01\right) \mathrm{d}\right)$ Photograph of HA-JR2EK (left) and HA-BCN hydrogels that have been incubated with JR2KK-Cy5 for 24 hours and sequentially have been incubated in PBS for 7 days.
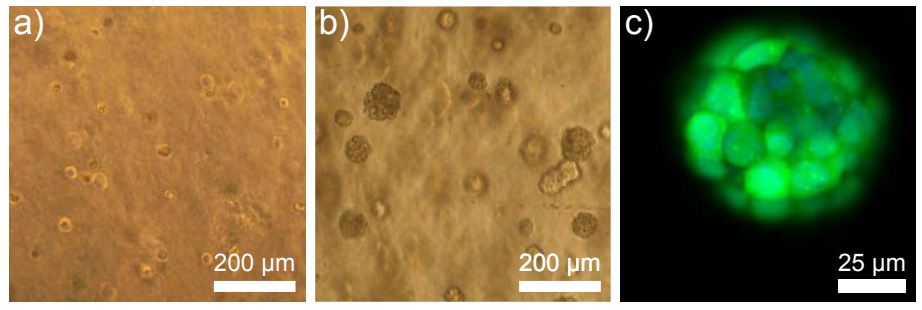

Figure 6.11: a) HepG2 cultured for 1 day and b) 7 days, showing the formation of spheroids. c) Spheroid stained with a Live/Dead assay and Hoechst 33342.

are unable to fold, e.g. by replacing all Ala residues to its enantiomer d-Ala. If a significant decrease in uptake should be seen with this d-Ala-JR2KK-Cy5, it would confirm that heterodimerization aid in the uptake of JR2KK-Cy5.

The accelerated gelation kinetics seen at $37{ }^{\circ} \mathrm{C}$ in combination with the bio-orthogonal SPAAC reaction makes the hydrogel interesting to be used as a scaffold for cell encapsulation and 3D cell culture. To examine the possibility to use the hydrogel for this purpose, HepG2 cells were encapsulated in the 
hydrogels. As described in section 6.2, HepG2 are of interest for in vitro drug screening. The HepG2 cells showed high viability after encapsulation in the hydrogel. The HepG2 increased their cell density and formed spheroids over time (Figure 6.11a-c). The average spheroid diameter was in the $\approx 80 \mu \mathrm{m}$ range, indicating that the oxygen diffusion to the spheroid core was sufficient. ${ }^{156}$ These initial cell studies thus showed that the SPAAC reaction and the assembly of the hydrogel were bio-orthogonal and cell-friendly. 


\section{Chapter 7}

\section{Characterization techniques}

Perhaps the best description of a scientist is a person who still maintains the ability to connect facts, in the way only kids can, while playing with sophisticated instruments.

"Are You a Scientist or a Researcher?" - Article in Elsevier SciTech Connect

This chapter will describe some of the characterization techniques that have been employed in this thesis.

\subsection{Spectroscopic techniques}

\subsubsection{Absorption spectroscopy}

\section{Utilized in paper $I-V$}

Absorption spectroscopy is a technique that measures the amount of light that is absorbed any given wavelength. Molecular groups with delocalized electrons, such as aromatic rings and conjugated structures, are often able to absorb light at certain wavelengths. ${ }^{181}$ The part of the molecule responsible for the absorption is called a chromophore. As light is absorbed by the chromophore, electrons in a bonding or a non-bonding orbital are excited to an empty anti-bonding orbital. As the electron relaxes back to the ground state, the gained excitation-energy is released, either as kinetic energy (heat) or as a photon (fluorescence, see subsection 7.1.4). Light that passes through an absorbing sample will thus contain lesser energy than initially, which can be 
measured. In addition to absorption, scattering and reflection can also occur in certain samples that will affect the amount of light that are able to pass through a sample. As an example, scattering is the major contributor in the turbidity measurements conducted in paper III.

Absorbance (in non-scattering and non-reflecting samples) is defined as

$$
A=\log \left(\frac{I}{I_{0}}\right)
$$

where $I_{0}$ is the incident light and $I$ is the transmitted light. The concentration of a chromophore can related to the absorbance by applying the Beer-Lambert law

$$
A=l \cdot c \cdot \epsilon
$$

where $l$ is the optical path length, $c$ is the molar concentration of the sample and $\epsilon$ is the molar extinction coefficient. If the $\epsilon$ at a certain wavelength of the sample is known, it is possible to relate the absorbance to the concentration of the chromophore. For instance, the polypeptides used in paper I and II contains an aromatic Trp amino acid residue with $\epsilon_{280}=5690 \mathrm{M}^{-1} \mathrm{~cm}^{-1}$. By applying the Beer-Lambert law it is possible to accurately determine the polypeptide concentration. Furthermore, the fluorophore $\mathrm{Cy}-5$ used in paper I and V has a known molar extinction coefficient of $\epsilon_{650}=250000 \mathrm{M}^{-1} \mathrm{~cm}^{-1}$, making it easily detectable with absorption spectroscopy (Figure 7.1).

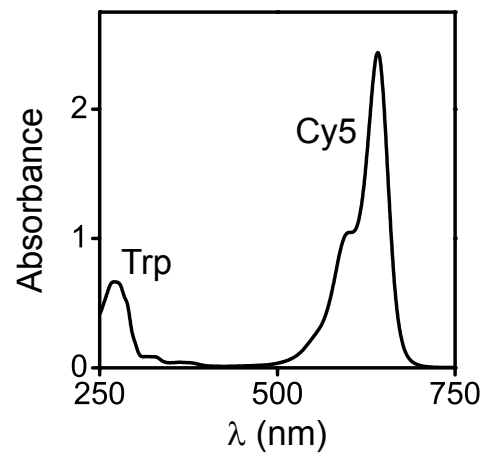

Figure 7.1: Absorbance spectra of the EV-Cy5 polypeptide used in paper I.

\subsubsection{Circular dichroism spectroscopy}

Utilized in paper $I-I V$

Circular dichroism (CD) spectroscopy is a widely used technique to study the conformation of peptides and proteins. Different secondary structures give rise to characteristic spectra that enables quantification of the conformation. $\mathrm{CD}$ is 
observed when an optically active substance (chiral) absorbs the left circularly polarized (LCP) and right circularly polarized (RCP) component of plane-polarized light slightly different. ${ }^{182}$ For peptides this mostly occurs in the far-UV region $(\lambda<250 \mathrm{~nm})$ and is caused by the chiral properties of the amide bonds in the peptide backbone. By applying equation 7.3, the difference in absorption of LCP and RCP light $(\Delta \mathrm{A})$ can be expressed as

$$
\Delta A=l \cdot c \cdot \Delta \epsilon
$$

where $l$ is the optical path length, $c$ is the molar concentration of the sample and $\Delta \epsilon$ is the difference in the molar extinction coefficients for LCP and RCP light, respectively. Due to the resulting elliptical shape of the light that has passed through a chiral sample, the $\Delta \mathrm{A}$ value is often converted into degrees of ellipticity $(\theta)$.

$$
\theta=\Delta A\left(\frac{\ln 10}{4}\right)\left(\frac{180}{\pi}\right)=32.98 \Delta A
$$

To make it possible to compare CD values for peptides and proteins of different lengths, $C D$ values are often converted to mean residue ellipticity (MRE)

$$
\mathrm{MRE}=\frac{100 \cdot \theta}{c \cdot n \cdot l}
$$

where $c$ is the molar concentration, $n$ is the number of amino acids and $l$ is the optical pathlength in $\mathrm{cm}$.

The absorption of the LCP and RCP light varies depending on the two dihedral angles $\phi$ and $\psi$ in the peptide bond. It is hence possible to extract information about the peptide secondary structure using CD spectroscopy. A spectrum of an $\alpha$-helix has a maximum around $192 \mathrm{~nm}$ and pronounced minima around 208 and $222 \mathrm{~nm}$ (Figure 7.2a). A $\beta$-sheet has a maximum around $195 \mathrm{~nm}$ and a minimum around $216 \mathrm{~nm}$ whereas a random coil has a minimum around $198 \mathrm{~nm}$.

CD measurements were extensively used in paper I to characterize the EKIV-polypeptide system. For instance, temperature scans were used to study the conformational transition from $\alpha$-helix to random coil upon heating (Figure 7.2b). During the transition an isobestic point at $203 \mathrm{~nm}$ can be noticed, indicating that the unfolding is discrete and that no other intermediate secondary structures were present. ${ }^{184}$ The changes in MRE of an $\alpha$-helix to random coil transition is most noticeable at $\lambda \approx 222 \mathrm{~nm}$. The CD-value at this wavelength is often plotted versus the temperature to depict the transition from $\alpha$-helix to random coil (Figure 7.2c). Another value of interest for coiled coils is the $\frac{\mathrm{MRE}_{222}}{\mathrm{MRE}_{208}}$ ratio. The ratio can give some indications on how well-defined the coiled coil structure is. ${ }^{90}$ As a rule of thumb, a ratio close to, or above 1 , indicates a well-defined coiled coil whereas lower ratios indicates a lesser well-defined coiled coil. If the ratio is below $\approx 0.8$ the polypeptides are probably either single $\alpha$-helices or random coils. 

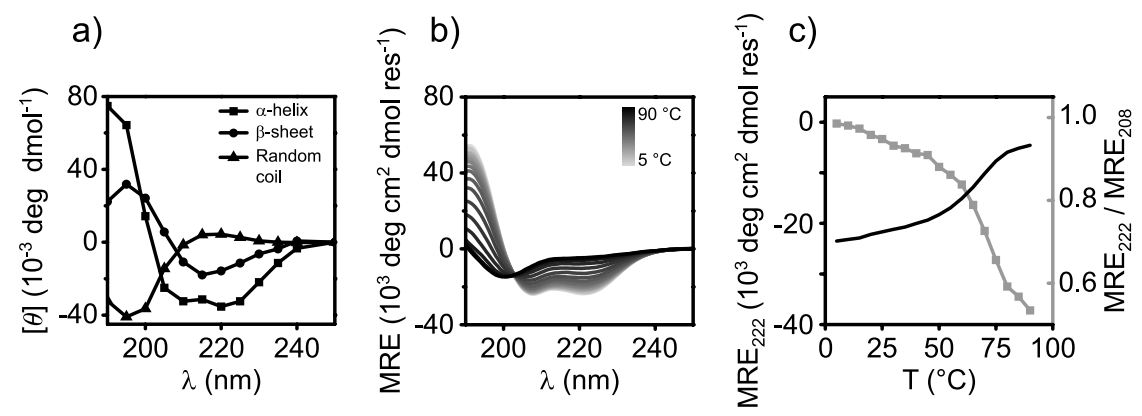

Figure 7.2: (a) Fasman standard curves of poly-Lysine, ${ }^{183}$ showing the CD-spectra of the three main secondary structures $\alpha$-helix, $\beta$-sheet and random coil. (b) Temperature scans for $\mathrm{EI} / \mathrm{KV}$ (c) $\mathrm{MRE}_{222}$ and $\frac{\mathrm{MRE}_{222}}{\mathrm{MRE}_{208}}$ for $\mathrm{EI} / \mathrm{KV}$ versus the temperature.

\subsubsection{Dynamic light scattering}

\section{Utilized in paper II-IV}

Dynamic light scattering (DLS), also known as photo correlation spectroscopy, is a technique that can be used to study size distributions of particles in solution. ${ }^{185}$ A monochromatic light is passed through a polarization filter into a sample. As the light hits the particles, the light will scatter in all directions (Rayleigh scattering) as long the particle size is small compared to the wavelength of the light. The scattered light is detected at a predetermined angle to the incident beam by a photo-multiplier and is analyzed by an autocorrelator. The measurement is repeated in very short intervals (ns) to enable detection of fluctuations in the scattered intensity caused by the movement of the particles (Brownian motion). Since smaller particles moves faster than larger particles, the movement can be related to the relative size and dynamics of the particles in the sample. The intensity autocorrelation function $g^{2}(\tau)$ is expressed as

$$
g^{2}(\tau)=\frac{\langle I(t) I(t+\tau)\rangle}{\langle I(t)\rangle^{2}}
$$

where $\tau$ is the lag time, and $I$ is the intensity of the light at a specific time point. At short lag times there will be almost no differences between $I(t)$ and $I(t+\tau)$, whereas for longer lag times there will be. The signal decays exponentially with increasing lag time as it will be lesser correlation between $I(t)$ and $I(t+\tau)$. Furthermore, $g^{2}(\tau)$ is often related to the field autocorrelation function $\left(g^{1}(\tau)\right)$ by the Siegert relation

$$
g^{2}(\tau)=B+\beta\left[g^{1}(\tau)\right]^{2}
$$

where $B$ is the baseline (often close to 1 in modern DLS-systems) and $\beta$ is a constant depending on the instruments light scattering setup. 


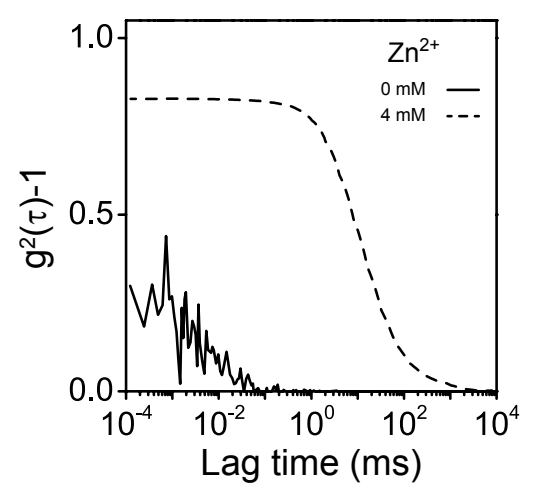

Figure 7.3: Normalized autocorrelation functions for $\mathrm{JR}_{2} \mathrm{EC}_{2}(50 \mu \mathrm{M}$ with and without 4 $\mathrm{mM} \mathrm{Zn^{2+ }}$.

An example of data obtained by DLS can be seen in Figure 7.3. As shown in paper III, when JR2EC 2 is subjected to $\mathrm{Zn}^{2+}$ it will self-assemble into large assemblies of superstructures. This event can be followed by DLS. Without $\mathrm{Zn}^{2+}, \mathrm{JR}_{2} \mathrm{EC}_{2}$ is unfolded and hence in the monomeric form. Since JR2EC 2 is a relative small molecules $(\mathrm{Mw} \approx 9000 \mathrm{Da})$, the autocorrelation function will decay exponentially at short lag times. In contrast, when $\mathrm{JR}_{2} \mathrm{EC}_{2}$ is subjected to $\mathrm{Zn}^{2+}$ and the larger assemblies form, the autocorrelation function will decay at longer lag times.

\subsubsection{Fluorescence spectroscopy}

\section{Utilized in paper I, III}

In fluorescence spectroscopy the spontaneous emission of photons from fluorescent molecules is measured (Figure 7.4a). ${ }^{186}$ When a molecule is absorbing light it will go into an excited electronic state. Surrounding molecules will collide with the excited molecule, and as it collides non-radiative energy will be given up to allow an excited electron to go to a lower vibrational level. As the electron reaches the lowest vibrational level, the surrounding molecules might not be able to accept the energy needed to go from the excited state to the ground state right away. In such cases, it is a chance that spontaneous emission occurs and the energy is emitted as a photon. Furthermore, the emitted photon has always lower energy (higher $\lambda$ ) compared to the photon used for excitation (Figure 7.4b). 


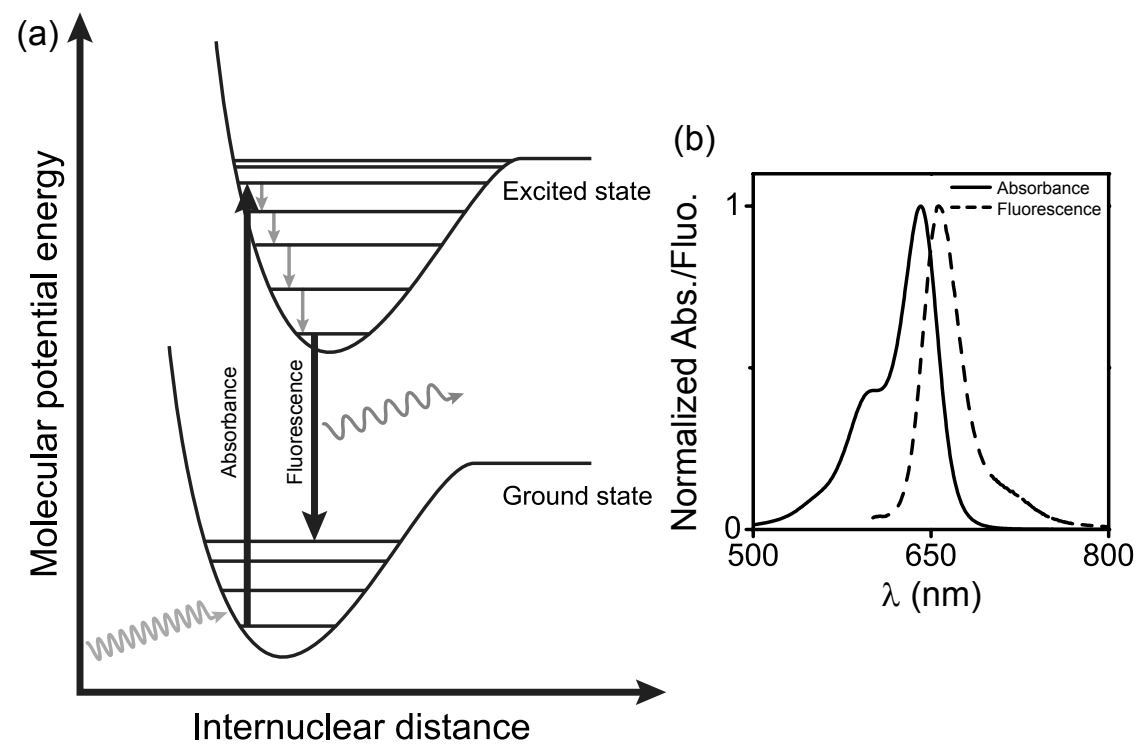

Figure 7.4: a) As a molecule is absorbing light it will gain potential energy and go into an excited state. Non-radiative energy will be given to the surroundings as it is relaxing to a lower vibrational level. A spontaneous emission of light can occur when the molecule relaxes back to the ground state. b) Absorbance- and resulting fluorescence spectra of the fluorophore Cy5.

\subsubsection{Fourier transform nuclear magnetic resonance spectroscopy}

\section{Utilized in paper II,IV}

Nuclear magnetic resonance (NMR) spectroscopy is one of the main characterization techniques used in organic chemistry. NMR can give structural data of molecules, for instance allowing spectral confirmation that a synthesis step has occurred. The technique relies on the magnetic moment of nuclei of some atoms, often referred to as nuclear spin (I) ${ }^{27}$ Due to their spherical charge distribution, isotopes that have a nucleus with $\mathrm{I}=\frac{1}{2}$ are the most useful in NMR spectroscopy. Therefore, the isotopes ${ }^{1} \mathrm{H}$ and ${ }^{13} \mathrm{C}$ are commonly used to assess NMR spectra of organic molecules. When an external magnetic field $\left(\mathrm{B}_{0}\right)$ is applied, nuclei with $\mathrm{I}=\frac{1}{2}$ can be in one of two different spin states, either the $\alpha$-spin state (lower energy) or the $\beta$-spin state (higher energy), which has an energy difference of $\Delta \mathrm{E}$ (Figure 7.5). By applying a pulse of energies of different frequencies, it is possible for a nucleus in the $\alpha$-spin state to change to the $\beta$-spin state if the exact $\Delta \mathrm{E}$ is meet (the nuclei is in resonance). As the pulse wears off, nuclei transitioning from $\beta \longrightarrow \alpha$ can be detected. The nuclei at different molecular environments will have different $\Delta \mathrm{E}$ and thus resonate at different frequencies (chemical shift, $\delta$ ). Furthermore, spin-spin coupling will 
occur that splits signal into several peaks. The different chemical shifts and spin-spin couplings are in turn used to assess the structure of a molecule.
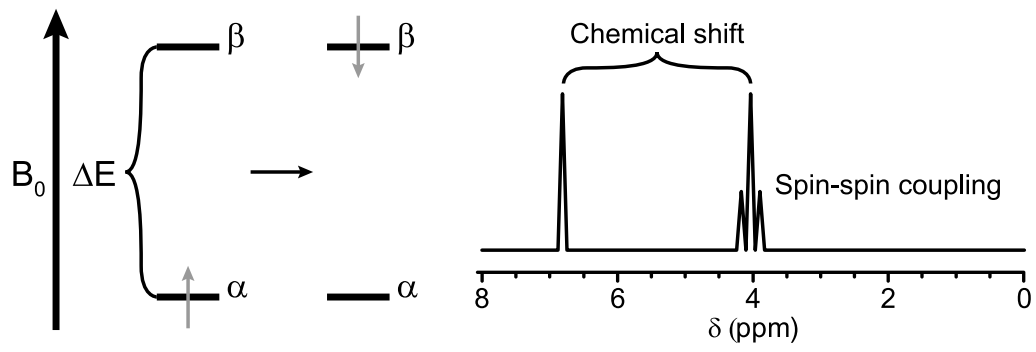

Figure 7.5: Schematic representation of the theory behind NMR.

\subsection{Microscopic techniques}

\subsubsection{Optical- and fluorescence microscopy}

\section{Utilized in paper II-III}

In optical- and fluorescence microscopy photons are used to visualize a sample. A simplified set-up of a conventional optical transmission microscope is demonstrated in Figure 7.6. Light is passed through a condenser lens to focus the light onto the sample. The transmitted light is sequentially passed through objective lenses that collects the light and magnifies the view of the sample. The light is guided to a detector such as a conventional eyepiece with ocular lenses or a CCD-camera. In fluorescence microscopy a slightly different set-up is used. Here light at specific wavelengths are used to excite incorporated fluorescent probes. In turn, the resulting emission is detected and visualized as an image.

Conventional optical microscopes have many great advantages such as easy operation and relative low cost. However, the main drawback is the limited spatial resolution. The limitation is mainly due to the wavelength of visible light, but also the material and the numerical aperture of the used objective lenses. Ernest Abbe, a microscopist in the 19th century, calculated already in 1883 that it would not be possible to increase the spatial resolution of a light-based microscope beyond $0.2 \mu \mathrm{m}$ due to these limitations. ${ }^{187}$ Using fluorophores it is however today possible go surpass this resolution limit, e.g. as shown by the 2014 Nobel Prize laureates in Chemistry. ${ }^{188}$ 


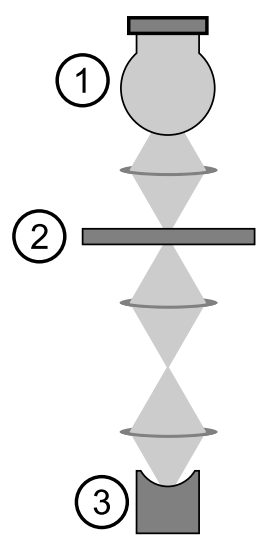

Optical

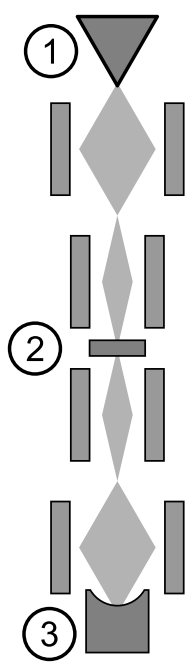

TEM



SEM

Figure 7.6: Simplified illustrations of a conventional optical transmission microscope, a conventional transmission electron microscope (TEM) and a scanning electron microscope (SEM) setup, showing the relative position of 1) Light/Electron source 2) Sample position and 3) Detector.

\subsubsection{Transmission electron microscopy}

\section{Utilized in paper III}

In transmission electron microscopy (TEM) electrons are used instead of photons. An electron gun produces a high energy electron beam that is focused onto the sample using either electrostatic or electromagnetic lenses (Figure 7.6). ${ }^{189}$ Electrons that are passed through the sample will contain structural information. Subsequently, the information is magnified by objective lenses and projected onto a fluorescent screen or a CCD camera system coated with phosphor to convert the electrons to visible photons.

When electrons are used instead of photons, a much higher resolution can be obtained. As an example, by using electrons with an energy of $200 \mathrm{keV}$ it is, in theory, possible to get a spatial resolution of $\approx 1 \mathrm{pm}$. The practical resolution is, however, closer to $\approx 0.1 \mathrm{~nm}$ due to limitations in the objective lens system of the TEM. In addition, high resolution TEM is not easily obtained for biological structures due to degradation caused by high energy electrons. Furthermore, as compared to many metals, thin biological samples do often show low contrast in TEM. The reason for this is that biological samples are composed of low electron density elements, such as carbon, nitrogen and oxygen. Electrons from the electron beam interact less with such elements compared to high electron 
density elements. ${ }^{190}$ Higher electron density elements, such as gold, has more electrons per atom that can interact and scatter the incoming electron beam. Areas with such elements in a sample will appear darker (in bright field mode) and hence give more contrast. By chemical staining with high electron density elements, such as tungsten and uranium, and/or using lower electron beam energies it is possible to enhance the contrast of biological structures. In paper III, TEM was used to visualize the assembled superstructures with encapsulated gold nanoparticles (Figure 6.5b). The thickness of the superstructures and possibly the incorporation of $\mathrm{Zn}^{2+}$ was enough to get sufficient contrast without use of any additional chemical staining. Furthermore, since the higher electron density of gold compared to the polypeptide, the encapsulated gold nanoparticles could easily be distinguished.

\subsubsection{Scanning electron microscopy}

\section{Utilized in paper II, III, V}

In scanning electron microscopy (SEM) an electron beam is used, as in TEM, to visualize the sample. One of the main differences between TEM and SEM is that in the latter the sample is scanned with a focused electron beam. The interactions between the electrons and the sample can give information about various features, such as morphology and chemical composition.

As in TEM, SEM is capable of creating high spatial resolution (approximately 1 $\mathrm{nm}$ ) images of a sample. Some drawbacks do, however, exist when imaging biological samples with SEM. In conventional SEM, samples must be electrically conductive and grounded. Biological samples are often not conductive, and hence must be sputter coated with an electrically conductive layer (e.g. gold, platinum or tungsten) and/or be impregnated with osmium to increase the bulk conductivity. Furthermore, samples must be completely dry and able to withstand the low pressure in the sample chamber. It is therefore common to chemically fixate the morphology of a biological structure with e.g. glutaraldehyde. Using increased concentrations of ethanol and hexamethyldisilazane it is possible to chemically dry the sample if air drying is not sufficient.

\subsection{Other techniques}

\subsubsection{Cell viability studies}

The viability of cells can be evaluated in many different ways. One of the simplest is to examine the morphology of the cells and the cell density over time using optical microscopy. A reduced cell density over time indicates a 
non-viable cell population. Other more elaborate methods are to use different colorimetric assays that can detect e.g. metabolic activity or dead cells. A very common and quick assay used to evaluate the viability of cells is a so called Live/Dead assay. ${ }^{191}$ The assay is based on the cell membranes permeability and esterase activity. The non-fluorescent acetomethoxy derivate of calcein (calcein-AM) can be transported through the cellular membrane of living cells. In the cell, esterases convert calcein-AM into calcein. When calcein complex intercellular $\mathrm{Ca}^{2+}$ it fluorescence in green, which in turn can be detected in a fluorescence microscope. Calcein-AM can thus be used to determine if a cell is alive. In contrast, ethidium homodimer-1 (EthD-1) can be used to determine if a cell is dead. EthD-1 is on its own weakly fluorescent. However, when EthD-1 binds to DNA the fluorescence will increase and emit in red. In addition, EthD-1 cannot go through the cellular membrane, meaning that it cannot be taken up by living cells. Dead cells will, however, have disrupted membranes and thus EthD-1 will be able to bind to the DNA of these cells.

\subsubsection{Oscillatory rheology}

Utilized in paper II, IV, V

Rheology is defined as the study of deformations and flow of matter upon an applied force. ${ }^{192}$ Fluids have irreversible deformation (flow) as their structure do not go back to the original state when the force is removed. In contrast, solids have reversible deformation (elasticity) as their structure goes back to the original state when the force is removed. However, many materials show both a fluid (viscous) and elastic behavior, often referred to as being viscoelastic. The viscoelastic behavior of e.g. hydrogels can be measured using oscillatory rheology, as done in paper II, IV and V. In oscillatory rheology the sample is exposed to a sinusoidal strain $(\gamma$, Equation 7.8) at an angular frequency $(\omega)$ and the resulting stress ( $\sigma$, Equation 7.9) is measured.

$$
\begin{gathered}
\gamma=\gamma_{0} \sin \omega t \\
\sigma=\sigma_{0} \sin (\omega t+\delta)
\end{gathered}
$$

For an elastic material the sinusoidal strain and stress response will be in phase $\left(\delta=0^{\circ}\right)$, whereas in a viscous material the sinusoidal strain and corresponding stress response will be out of phase by $90^{\circ}$ (Figure 7.7). A viscoelastic material will show a phase angle $(\delta)$ between 0 to $90^{\circ}$. Furthermore, from equation 7.9 the storage modulus (G', Equation 7.10) and the loss modulus (G”, Equation 7.11) of a material can be obtained.

$$
\begin{aligned}
G^{\prime} & =\frac{\sigma_{0}}{\gamma_{0}} \cos \delta \\
G^{\prime \prime} & =\frac{\sigma_{0}}{\gamma_{0}} \sin \delta
\end{aligned}
$$


The G' represents the elastic portion of a material, whereas G" represent the viscous portion. The two quantities are some of the most commonly used values when describing the rheological properties of a hydrogel. G' and G" can in turn be plotted against a myriad of quantities, such as time, temperature and angular frequency.

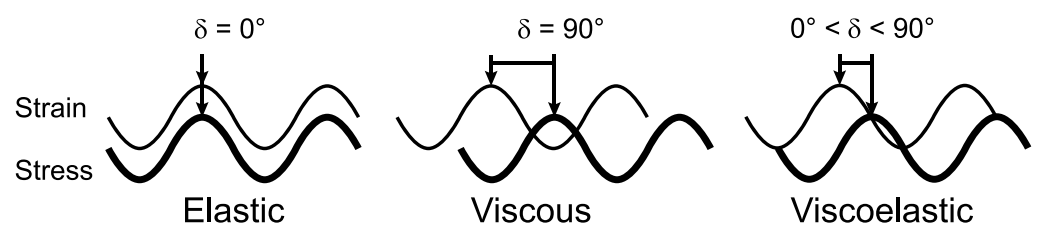

Figure 7.7: The difference in stress response between an elastic, a viscous and a viscoelastic material upon strain. 


\section{Chapter 8}

\section{Summary of papers}

Five papers are included in this thesis. Three are published in peer-reviewed journals, one is submitted and one is in manuscript. This chapter includes a short summary of each paper included and lists where the main results and discussions relating to the paper can be found in this thesis. 


\section{Paper I}

\section{Self-sorting heterodimeric coiled coil peptides with defined and tuneable self-assembly properties}

C. Aronsson, S. Dånmark, F. Zhou, P. Öberg, K. Enander, H. Su, D. Aili

Scientific Reports 2015, 5:14063

The paper describes the design of four polypeptides that can heterodimerize into parallel coiled coils. The four polypeptides can be mix-and-matched to form four different heterodimers with $\mathrm{K}_{\mathrm{d}}$ ranging from $\mu \mathrm{M}$ to $<\mathrm{nM}$. In addition, the paper shows that the polypeptides self-sort into two out of four heterodimers when all are present in a solution. The difference in affinities and the self-sorting capability make these polypeptides highly attractive supramolecular building blocks when creating e.g. supramolecular biomaterials.

Materials, results and discussions relating to this paper can mainly be found in the following section(s) of this thesis:

Section 4.1 


\title{
Paper II
}

\section{Tailoring supramolecular peptide-poly(ethylene gly- col) hydrogels by coiled coil self-assembly and self- sorting}

\author{
S. Dånmark ${ }^{\dagger}$, C. Aronsson ${ }^{\dagger}$, D. Aili \\ $\dagger$ Equal contribution
}

Biomacromolecules 2016, 17(6):2260-2267

The paper show-case the possibility to control the mechanical properties of hydrogels using coiled coil polypeptides with different affinity for heterodimerization. Four different polypeptides were conjugated to four-armed star-shaped poly(ethylene glycol) (PEG). The different combinations of polypeptide-PEG conjugates assemble into polymeric networks and hydrogels with distinct different thermal stabilities, supramolecular- and rheological properties. The paper does also show the possibility to disassemble and re-assemble polymeric networks using the self-sorting phenomenon of the polypeptides.

Materials, results and discussions relating to this paper can mainly be found in the following section(s) of this thesis:

\section{Section 4.1}

Subsection 5.2.1

Section 6.2 


\section{Paper III}

\section{Zinc-triggered hierarchical self-assembly of fibrous helix-loop-helix peptide superstructures for con- trolled encapsulation and release}

C. Aronsson ${ }^{\dagger}$, R. Selegård ${ }^{\dagger}$, D. Aili

$\dagger$ Equal contribution

Macromolecules 2016, 49(18):6997-7003

The paper describes a hierarchical self-assembly process of a $\mathrm{Zn}^{2+}$ responsive disulfide-linked helix-loop-helix polypeptide that selfassembles into superstructures. Furthermore, the self-assembly process is fully reversible and the superstructures can be disassembled upon removal of $\mathrm{Zn}^{2+}$ or by degradation of the polypeptide disulpfide bridge. In addition, the paper shows the possibility to both encapsulate and to release nanoparticles and drugs from the superstructures.

Materials, results and discussions relating to this paper can mainly be found in the following section(s) of this thesis:

\section{Section 4.2}

Subsection 6.3 


\section{Paper IV}

\section{Folding driven self-assembly of a stimuli-responsive peptide-hyaluronan hybrid hydrogel}

R. Selegård, C. Aronsson, C. Brommesson, S. Dånmark, D. Aili

\section{Submitted}

The paper describes a tunable supramolecular polypeptide-hyaluronic acid hydrogel that responds and self-assembles with $\mathrm{Zn}^{2+}$. The conjugated polypeptides homodimerizes into four-helix bundles upon addition of $\mathrm{Zn}^{2+}$, resulting in hydrogel formation. The assembly of the hydrogel is reversible as chelation of $\mathrm{Zn}^{2+}$ disassembles the hydrogel. Furthermore, incorporated hyaluronidase can be used to tune the degradation rate of the hydrogel. In addition, the hydrogel is evaluated as an in vitro model for scavenging of reactive oxygen species.

Materials, results and discussions relating to this paper can mainly be found in the following section(s) of this thesis:

\section{Section 4.2}

Subsection 5.2 .2

Subsection 6.4 


\section{Paper V}

\section{Supramolecular Functionalization and Tuning of Peptide Modified Bio-Orthogonally Crosslinked Hyaluronan-Poly(ethylene glycol) Hydrogels}

C. Aronsson ${ }^{\dagger}$, R. Selegård ${ }^{\dagger}$, J. Christoffersson, C-F. Mandenius, D. Aili

$\dagger$ Equal contribution

Manuscript

The paper describes a modular and tunable covalently crosslinked polypeptide-hyaluronic acid and poly(ethylene glycol) hydrogel. The hydrogel is assembled via a bio-orthogonal strain-promoted alkyneazide cycloaddition reaction, making the hydrogel suitable for 3D cell encapsulation and culture. Furthermore, the polypeptide allows for supramolecularly crosslinking and compositional post-modifications of the hydrogel.

Materials, results and discussions relating to this paper can mainly be found in the following section(s) of this thesis:

\section{Section 4.2}

Subsection 5.2 .2

Subsection 6.5 


\section{Chapter 9}

\section{Conclusions and future outlook}

My interest is in the future because I am going to spend the rest of my life there.

Charles Kettering

\subsection{Conclusions}

In this thesis, two different polypeptide-systems have been used to create functional polypeptide-hybrid biomaterials. In turn, the different polypeptide-hybrid biomaterials have been used to create modularly assembling and highly tunable structures. The main conclusions of each paper and the main overall conclusions of this thesis are summarized below.

\section{Paper I}

The four polypeptides constituting the EKIV-polypeptide system can form four different parallel heterodimers with large difference in affinities with $\mathrm{K}_{\mathrm{d}}$ ranging from $\mu \mathrm{M}$ to $\ll \mathrm{nM}$. In addition, the large difference in affinities allowed for a social nonintegrative self-sorting when all polypeptides were present in the same solution. The EKIV-polypeptide system thus constitute a very useful set of molecular building blocks, that can facilitate development of novel supramolecular biomaterials.

\section{Paper II}

A tunable and modular polypeptide-PEG hybrid system was developed. Using the four polypeptides of the EKIV-polypeptide system, polymeric networks have been created with distinct differences in e.g. thermal stabilities and 
rheological properties. Although only two out of four possible polypeptide-PEG combinations $\left(\mathrm{pEI}_{4} / \mathrm{pKI}_{4}\right.$ and $\mathrm{pEI}_{4} / \mathrm{pKV}_{4}$ ) were able to form hydrogels under the examined conditions, the two hydrogels had $G^{\prime}$ in a biological relevant range and showed typical rheological properties of supramolecular hydrogels such as shear-thinning and self-healing. Furthermore, due to the self-sorting capability of the polypeptides, it was possible to disassemble (and reassemble) the polymeric networks by adding nonpegylated polypeptides with higher affinity for heterodimerization. The EKIV-polypeptide system thus allows for a modular approach when fabricating hydrogels and where mechanical properties etc. can be tuned and optimized to specific biomaterial applications by means of polypeptide heterodimerization.

\section{Paper III}

By linking two JR2EC-polypeptides via a disulfide bridge, it was possible to trigger a hierarchical self-assembly process into spherical superstructures with $\mathrm{Zn}^{2+}$. The self-assembly process depended on the folding and the homoassociation of $\mathrm{JR}_{2} \mathrm{EC}_{2}$ and could be tuned by the $\mathrm{Zn}^{2+}$ concentration. Furthermore, the superstructures could be disassembled either by $\mathrm{Zn}^{2+}$ depletion or reducing the disulfide bridge. In addition, it was possible to both encapsulate and to release nanoparticles and molecules with the superstructures. The release-rate could be tuned by varying the $\mathrm{Zn}^{2+}$ concentration. The tunable self-assembly properties and possible release of compounds make this system interesting in both drug delivery and bioanalytical applications.

\section{Paper IV}

By conjugating the HLH-polypeptide JR2E to hyaluronic acid a $\mathrm{Zn}^{2+}$-responsive polypeptide-hyaluronic acid hybrid polymer was developed. $\mathrm{Zn}^{2+}$ triggers the self-assembly of hydrogels, which is caused by the folding and dimerization of the polypeptides into four-helix bundles. The mechanical properties, and the assembly and disassembly, could be tuned by changing the $\mathrm{Zn}^{2+}$ concentration. By encapsulating an enzyme that degrades hyaluronic acid, it was possible to tune the degradation rate of the hydrogel and thus also the release of encapsulated compounds. The $\mathrm{Zn}^{2+}$-containing hydrogels showed inhibitory effects on the ROS production of neutrophils, although $\mathrm{Zn}^{2+}$ probably was the major reason for this effect. All in all, the developed $\mathrm{HA} / \mathrm{Zn}^{2+}$-hydrogel system is a highly dynamic biomaterial that allow tunable release of encapsulated compounds.

\section{Paper V}

By conjugating the HLH-polypeptide JR2EK to a BCN-modified hyaluronic acid via SPAAC and by utilizing the remaining $\mathrm{BCN}$-groups for covalent crosslinking with a star-shaped azide-terminated PEG-molecule, a modular and tunable hydrogel system was developed. The mechanical properties could be tuned by varying the total polymer concentration or by exploiting the homodimerization of JR2EK. In addition, using the heterodimerization with the complementary 
polypeptide JR2KK, JR2EK could be used for non-covalent post-modification of the hydrogel. Furthermore, HepG2 cells showed high viability when encapsulated and cultured in the hydrogel. The developed HA/PEG-hybrid hydrogel is thus an interesting system for several biomaterial applications, such as $3 \mathrm{D}$ cell culture and tissue engineering.

\section{Overall conclusions of this thesis}

Dimerizing $\alpha$-helical polypeptides are very flexible and versatile supramolecular building blocks that can be used for fabrication of biomaterials with a wide range of tunable properties. Furthermore, the modular assembly strategies developed and explored in the thesis allows for a mix-and-match approach when fabricating a material.

\subsection{Future outlook}

Biomaterials that fully, or partly, rely on supramolecular interactions have without doubt a bright future in medicine. As exemplified in this thesis, dimerizing $\alpha$-helical polypeptides are flexible and versatile supramolecular building blocks that allow for tuning of the material properties and composition. The main focus of this thesis has been on the fabrication and the physiochemical properties of the actual material. It is not until now at the end of my PhD that the "bio"-part of the biomaterial has gained more attention. However, from the very beginning polymers that are considered to be biocompatible have been used and bio-orthogonal assembly strategies have been employed to ensure a solid foundation for future cell studies.

The modular assembly strategy that has been developed and employed in this thesis allows for a mix-and-match approach when fabricating a biomaterial. By expanding the library of polypeptide-hybrids and/or molecules with SPAAC reactive groups, more complex and even more ECM-like biomaterials could be fabricated. Examples of interesting and possible molecules to include in such a library are different biopolymers such as collagen and chitosan, or different types of PEG structures. Furthermore, cell adhesion motifs, such as RGD and IKVAV, and growth factors could also be incorporated in a modular fashion using for instance a similar approach as the Cy5-functionalization used in paper $\mathrm{V}$. The modular approach hence allows for a high degree of customization of the final biomaterial properties and can be modularly designed to fit the requirements of the end-application.

Even though a relatively high tunability can be achieved with the current polypeptides, point mutations in the amino acid sequences can be made to allow further fine-tuning. As an example, the EKIV-polypeptide library can be extended with even more polypeptides by e.g. changing the Ser at the $b$ positions in the EV and KV to Ala, or changing a single Ile or Val in the hydrophobic core by another amino acid. Such changes would create new 
polypeptides that could be used in combination with the already existing EKIV-polypeptides. The new polypeptides could then be used to tune the assembly of polypeptide-hybrid biomaterials even further. 


\section{Bibliography}

${ }^{1}$ MarketsandMarkets. Biomaterials market by type of material (metallic, ceramic, polymers, natural biomaterials) \& application (cardiovascular, orthopedic, dental, plastic surgery, wound healing, neurology, tissue engineering, ophthalmology) - global forecast to 2020. Report, 2015.

${ }^{2}$ Fergal J. O’Brien. Biomaterials; scaffolds for tissue engineering. Mater. Today, 14(3):88-95, 2011.

${ }^{3}$ Buddy D. Ratner, Allan S. Hoffman, Frederick J. Schoen, and Jack E. Lemons. Biomaterials Science An Introduction to Materials in Medicine. Elsevier Science, Saint Louis, 2014.

${ }^{4}$ Larry L. Hench. Bioceramics. F. Am. Ceram. Soc., 81(7):1705-1728, 1998.

${ }^{5}$ Larry L. Hench and Julia M. Polak. Third-generation biomedical materials. Science, 295(5557):1014, 2002

${ }^{6}$ Wanpeng Cao and Larry L. Hench. Bioactive materials. Ceram. Int., 22(6):493-507, 1996.

${ }^{7}$ U. Heise, J. F. Osborn, and F. Duwe. Hydroxyapatite ceramic as a bone substitute. Int. Orthop., 14(3):329-338, 1990.

${ }^{8}$ C. A. van Blitterswijk, J. J. Grote, W. Kuijpers, W. Th Daems, and K. de Groot. Macropore tissue ingrowth: a quantitative and qualitative study on hydroxyapatite ceramic. Biomaterials, 7(2):137$143,1986$.

${ }^{9}$ H. Oonishi, M. Yamamoto, H. Ishimaru, E. Tsuji, S. Kushitani, M. Aono, and Y. Ukon. The effect of hydroxyapatite coating on bone growth into porous titanium alloy implants. Bone foint f., 71-B(2):213, 1989.

${ }^{10}$ C. C. Chu. An in-vitro study of the effect of buffer on the degradation of poly(glycolic acid) sutures. f. Biomed. Mater. Res., 15(1):19-27, 1981.

${ }^{11}$ D. F. Williams. Biodegradation of surgical polymers. F. Mater. Sci., 17(5):1233-1246, 1982.

${ }^{12}$ H. J. Krause, A. Schwarz, and P. Rohdewald. Polylactic acid nanoparticles, a colloidal drug delivery system for lipophilic drugs. Int. F. Pharm., 27(2):145-155, 1985.

${ }^{13}$ Alan Smith and lan M. Hunneyball. Evaluation of poly(lactic acid) as a biodegradable drug delivery system for parenteral administration. Int. J. Pharm., 30(2):215-220, 1986.

${ }^{14}$ Anika M. Jonker, Saskia A. Bode, Addie H. Kusters, Jan C. M. van Hest, and Dennis W. P. M. Löwik. Soft peg-hydrogels with independently tunable stiffness and rgds-content for cell adhesion studies. Macromol. Biosci., 15(10):1338-1347, 2015.

${ }^{15}$ Ali Adem Bahar and Dacheng Ren. Antimicrobial peptides. Pharmaceuticals, 6(12):1543-1575, 2013.

${ }^{16}$ Staffan Dånmark, Christopher Aronsson, and Daniel Aili. Tailoring supramolecular peptide-poly(ethylene glycol) hydrogels by coiled coil self-assembly and self-sorting. Biomacromolecules, 17(6):2260-2267, 2016.

${ }^{17}$ Matthew J. Webber, Eric A. Appel, E. W. Meijer, and Robert Langer. Supramolecular biomaterials. Nat. Mater., 15(1):13-26, 2016.

${ }^{18}$ D. N. Woolfson. Building fibrous biomaterials from alpha-helical and collagen-like coiled-coil peptides. Biopolymers, 94(1):118-27, 2010. 
${ }^{19}$ Johannes van der Waals. Over de continuiteit van de gas- en vloeistoftoestand. Thesis, Hoogeschool te Leiden, 1873.

${ }^{20}$ Charles J. Pedersen. Cyclic polyethers and their complexes with metal salts. J. Am. Chem. Soc., 89(26):7017-7036, 1967.

${ }^{21}$ Jean-Marie Lehn. Supramolecular chemistry-scope and perspectives molecules, supermolecules, and molecular devices (nobel lecture). Angew. Chem. Int. Edit., 27(1):89-112, 1988.

${ }^{22}$ Donald J. Cram. The design of molecular hosts, guests, and their complexes (nobel lecture). Angew. Chem. Int. Edit., 27(8):1009-1020, 1988.

23 ”The Nobel Prize in Chemistry 1987”. Nobelprize.org. Web 15 Apr 2016.

24 "The Nobel Prize in Chemistry 2016". Nobelprize.org. Web 27 Oct 2016.

${ }^{25}$ J. W. Steed, D. R. Turner, and K. J. Wallace. Core Concepts in Supramolecular Chemistry and Nanotechnology. Wiley, 1 edition, 2007.

${ }^{26}$ M. Clugston and R. Flemming. Advanced Chemistry. OUP Oxford, 2000.

${ }^{27}$ P. Atkins and J. De Paula. Atkins' Physical Chemistry. Oxford University Press, 8 edition, 2006.

${ }^{28}$ George M. Whitesides and Mila Boncheva. Beyond molecules: Self-assembly of mesoscopic and macroscopic components. Proc. Natl. Acad. Sci. USA, 99(8):4769-4774, 2002.

${ }^{29}$ Olivier Toussaint and Eric D. Schneider. The thermodynamics and evolution of complexity in biological systems. Comp. Biochem. Physiol. A: Mol. Integr. Physiol., 120(1):3-9, 1998.

${ }^{30}$ José Nelson Onuchic, Zaida Luthey-Schulten, and Peter G. Wolynes. Theory of protein folding: The energy landscape perspective. Annu. Rev. Phys. Chem., 48(1):545-600, 1997.

${ }^{31}$ Luis A. Marky and Kenneth J. Breslauer. Calculating thermodynamic data for transitions of any molecularity from equilibrium melting curves. Biopolymers, 26(9):1601-1620, 1987.

${ }^{32}$ SA Bobrovnik. Determination of antibody affinity by elisa. theory. F. Biochem. Biophys. Methods, 57(3):213-236, 2003.

${ }^{33}$ D. J. Oshannessy, M. Brighamburke, K. K. Soneson, P. Hensley, and I. Brooks. Determination of rate and equilibrium binding constants for macromolecular interactions using surface plasmon resonance: Use of nonlinear least squares analysis methods. Anal. Biochem., 212(2):457-468, 1993.

${ }^{34}$ Giorgio Pochetti and Roberta Montanari. Isothermal titration calorimetry to determine the association constants for a ligand bound simultaneously to two specific protein binding sites with different affinities. Protoc. Exch., 2012.

${ }^{35}$ Marina M. Safont-Sempere, Gustavo Fernández, and Frank Würthner. Self-sorting phenomena in complex supramolecular systems. Chem. Rev., 111(9):5784-5814, 2011.

${ }^{36}$ Sabine Himmelein, Vanessa Lewe, Marc C. A. Stuart, and Bart Jan Ravoo. A carbohydrate-based hydrogel containing vesicles as responsive non-covalent cross-linkers. Chemi. Sci., 5(3):1054-1058, 2014.

${ }^{37}$ Charles M. Rubert Perez, Leslie A. Rank, and Jean Chmielewski. Tuning the thermosensitive properties of hybrid collagen peptide-polymer hydrogels. Chem. Commun., 50(60):8174-8176, 2014.

${ }^{38}$ Patrick J. Stahl, Nicole H. Romano, Denis Wirtz, and S. Michael Yu. Peg-based hydrogels with collagen mimetic peptide-mediated and tunable physical cross-links. Biomacromolecules, 11(9):2336-2344, 2010.

${ }^{39}$ Chunyu Xu, Victor Breedveld, and Jindřich Kopeček. Reversible hydrogels from self-assembling genetically engineered protein block copolymers. Biomacromolecules, 6(3):1739-1749, 2005.

${ }^{40}$ Wendy A. Petka, James L. Harden, Kevin P. McGrath, Denis Wirtz, and David A. Tirrell. Reversible hydrogels from self-assembling artificial proteins. Science, 281(5375):389, 1998.

${ }^{41}$ Rui Li, Conor C. Horgan, Benjamin Long, Alexandra L. Rodriguez, Lauren Mather, Colin J. Barrow, David R. Nisbet, and Richard J. Williams. Tuning the mechanical and morphological properties of self-assembled peptide hydrogels via control over the gelation mechanism through regulation of ionic strength and the rate of ph change. RSC Adv., 5(1):301-307, 2015. 
${ }^{42}$ Shibaji Basak, Jayanta Nanda, and Arindam Banerjee. Multi-stimuli responsive self-healing metallo-hydrogels: tuning of the gel recovery property. Chem. Comm., 50(18):2356-2359, 2014.

${ }^{43}$ Lin Chen, Guillaume Pont, Kyle Morris, Gudrun Lotze, Adam Squires, Louise C. Serpell, and Dave J. Adams. Salt-induced hydrogelation of functionalised-dipeptides at high ph. Chem. Commun., 47(44):12071-12073, 2011.

${ }^{44}$ Akira Harada, Ryosuke Kobayashi, Yoshinori Takashima, Akihito Hashidzume, and Hiroyasu Yamaguchi. Macroscopic self-assembly through molecular recognition. Nat. Chem., 3(1):34-37, 2011.

${ }^{45}$ Abu Bin Imran, Kenta Esaki, Hiroaki Gotoh, Takahiro Seki, Kohzo Ito, Yasuhiro Sakai, and Yukikazu Takeoka. Extremely stretchable thermosensitive hydrogels by introducing slide-ring polyrotaxane cross-linkers and ionic groups into the polymer network. Nat. Commun., 5:5124, 2014.

${ }^{46}$ Kerh Li Liu, Zhongxing Zhang, and Jun Li. Supramolecular hydrogels based on cyclodextrinpolymer polypseudorotaxanes: materials design and hydrogel properties. Soft Matter, 7(24):1129011297, 2011.

${ }^{47}$ Niels Holten-Andersen, Aditya Jaishankar, Matthew Harrington, Dominic E. Fullenkamp, Genevieve DiMarco, Lihong He, Gareth H. McKinley, Phillip B. Messersmith, and Ka Yee C. Lee. Metal-coordination: Using one of nature's tricks to control soft material mechanics. f. Mater. Chem. B, 2(17):2467-2472, 2014.

${ }^{48}$ Niels Holten-Andersen, Matthew J. Harrington, Henrik Birkedal, Bruce P. Lee, Phillip B. Messersmith, Ka Yee C. Lee, and J. Herbert Waite. ph-induced metal-ligand cross-links inspired by mussel yield self-healing polymer networks with near-covalent elastic moduli. Proc. Natl. Acad. Sci. USA, 108(7):2651-2655, 2011

${ }^{49}$ Mark Burnworth, Liming Tang, Justin R. Kumpfer, Andrew J. Duncan, Frederick L. Beyer, Gina L. Fiore, Stuart J. Rowan, and Christoph Weder. Optically healable supramolecular polymers. Nature, 472(7343):334-337, 2011.

${ }^{50}$ Chuang Li, Alan Faulkner-Jones, Alison R. Dun, Juan Jin, Ping Chen, Yongzheng Xing, Zhongqiang Yang, Zhibo Li, Wenmiao Shu, Dongsheng Liu, and Rory R. Duncan. Rapid formation of a supramolecular polypeptide-dna hydrogel for in situ three-dimensional multilayer bioprinting. Angew. Chem. Int. Edit., 54(13):3957-3961, 2015.

${ }^{51}$ Ingrid Wagner and Hans Musso. New naturally occurring amino acids. Angew. Chem. Int. Edit., 22(11):816-828, 1983.

${ }^{52}$ IUPAC-IUB Joint Commission on Biochemical Nomenclature (JCBN). Nomenclature and symbolism for amino acids and peptides. Eur. f. Biochem., 138(1):9-37, 1984.

${ }^{53}$ Mike Williamson. How proteins work. Garland Science, New York, 2012.

${ }^{54}$ R. Zwanzig, A. Szabo, and B. Bagchi. Levinthal's paradox. Proc. Natl. Acad. Sci. USA, 89(1):20-22, 1992.

${ }^{55}$ Joseph D. Bryngelson, José Nelson Onuchic, Nicholas D. Socci, and Peter G. Wolynes. Funnels, pathways, and the energy landscape of protein folding: A synthesis. Proteins, 21(3):167-195, 1995.

${ }^{56}$ Gergely Agócs, Bence T Szabó, Gottfried Köhler, and Szabolcs Osváth. Comparing the folding and misfolding energy landscapes of phosphoglycerate kinase. Biophys. F., 102(12):2828-2834, 2012.

${ }^{57}$ Ying-Ching Yu, Peter Berndt, Matthew Tirrell, and Gregg B. Fields. Self-assembling amphiphiles for construction of protein molecular architecture. F. Am. Chem. Soc., 118(50):12515-12520, 1996.

${ }^{58}$ Jeffrey D. Hartgerink, Elia Beniash, and Samuel I. Stupp. Self-assembly and mineralization of peptide-amphiphile nanofibers. Science, 294(5547):1684, 2001.

${ }^{59}$ J. K. Kretsinger, L. A. Haines, B. Ozbas, D. J. Pochan, and J. P. Schneider. Cytocompatibility of self-assembled beta-hairpin peptide hydrogel surfaces. Biomaterials, 26(25):5177-86, 2005.

${ }^{60}$ Lisa Haines-Butterick, Karthikan Rajagopal, Monica Branco, Daphne Salick, Ronak Rughani, Matthew Pilarz, Matthew S. Lamm, Darrin J. Pochan, and Joel P. Schneider. Controlling hydrogelation kinetics by peptide design for three-dimensional encapsulation and injectable delivery of cells. Proc. Natl. Acad. Sci. USA, 104(19):7791-7796, 2007. 
${ }^{61}$ S Zhang, T Holmes, C Lockshin, and A Rich. Spontaneous assembly of a self-complementary oligopeptide to form a stable macroscopic membrane. Proc. Natl. Acad. Sci. USA, 90(8):3334-3338, 1993.

62 Jaclyn Raeburn, Guillaume Pont, Lin Chen, Yann Cesbron, Raphael Levy, and Dave J. Adams. Fmocdiphenylalanine hydrogels: understanding the variability in reported mechanical properties. Soft Matter, 8(4):1168-1174, 2012.

${ }^{63}$ Mona Widhe, Jan Johansson, My Hedhammar, and Anna Rising. Current progress and limitations of spider silk for biomedical applications. Biopolymers, 97(6):468-478, 2012.

${ }^{64} \mathrm{~W}$. T. Astbury and A. Street. X-ray studies of the structure of hair, wool, and related fibres. i. general. Philos. T. Roy. Soc. A., 230(681-693):75-101, 1932.

${ }^{65}$ Linus Pauling, Robert B. Corey, and H. R. Branson. The structure of proteins: Two hydrogenbonded helical configurations of the polypeptide chain. Proc. Natl. Acad. Sci. USA, 37(4):205-211, 1951.

${ }^{66}$ Imre Jákli, Imre G. Csizmadia, Szilard N. Fejer, Ödön Farkas, Bela Viskolcz, Svend J. Knak Jensen, and Andras Perczel. Helix compactness and stability: Electron structure calculations of conformer dependent thermodynamic functions. Chem. Phys. Lett., 563:80-87, 2013.

${ }^{67}$ D. A. D. Tarry and E. Suzuki. Intrachain potential energy of the $\alpha$-helix and of a coiled coil strand. Biopolymers, 7(2):189-197, 1969.

${ }^{68}$ D. N. Woolfson. The design of coiled-coil structures and assemblies. Adv. Protein Chem., 70:79-112, 2005.

${ }^{69}$ M. J. Pandya, G. M. Spooner, M. Sunde, J. R. Thorpe, A. Rodger, and D. N. Woolfson. Stickyend assembly of a designed peptide fiber provides insight into protein fibrillogenesis. Biochem., 39(30):8728-34, 2000.

${ }^{70}$ Eleanor F. Banwell, Edgardo S. Abelardo, Dave J. Adams, Martin A. Birchall, Adam Corrigan, Athene M. Donald, Mark Kirkland, Louise C. Serpell, Michael F. Butler, and Derek N. Woolfson. Rational design and application of responsive $\alpha$-helical peptide hydrogels. Nat. Mater., 8(7):596$600,2009$.

${ }^{71}$ Thomas H. Sharp, Marc Bruning, Judith Mantell, Richard B. Sessions, Andrew R. Thomson, Nathan R. Zaccai, R. Leo Brady, Paul Verkade, and Derek N. Woolfson. Cryo-transmission electron microscopy structure of a gigadalton peptide fiber of de novo design. Proc. Natl. Acad. Sci. USA, 109(33):13266-13271, 2012.

${ }^{72}$ Daniel Aili, Feng-I. Tai, Karin Enander, Lars Baltzer, and Bo Liedberg. Self-assembly of fibers and nanorings from disulfide-linked helix-loop-helix polypeptides. Angew. Chem. Int. Edit., 47(30):5554-5556, 2008.

${ }^{73}$ E. H. Egelman, C. Xu, F. DiMaio, E. Magnotti, C. Modlin, X. Yu, E. Wright, D. Baker, and V. P. Conticello. Structural plasticity of helical nanotubes based on coiled-coil assemblies. Structure, 23(2):280-289, 2015

${ }^{74}$ Chun Wang, Russell J. Stewart, and JindRich KopeCek. Hybrid hydrogels assembled from synthetic polymers and coiled-coil protein domains. Nature, 397(6718):417-420, 1999.

${ }^{75}$ Jiyuan Yang, Chunyu Xu, Chun Wang, and Jindřich Kopeček. Refolding hydrogels self-assembled from n-(2-hydroxypropyl)methacrylamide graft copolymers by antiparallel coiled-coil formation. Biomacromolecules, 7(4):1187-1195, 2006.

${ }^{76}$ Helena Gradišar, Sabina Božič, Tibor Doles, Damjan Vengust, Iva Hafner-Bratkovič, Alenka Mertelj, Ben Webb, Andrej Šali, Sandi Klavžar, and Roman Jerala. Design of a single-chain polypeptide tetrahedron assembled from coiled-coil segments. Nat. Chem. Biol., 9(6):362-366, 2013.

${ }^{77}$ Linus Pauling and Robert B. Corey. Compound helical configurations of polypeptide chains: Structure of proteins of the $\alpha$-keratin type. Nature, 171(4341):59-61, 1953.

${ }^{78}$ F. Crick. The packing of $\alpha$-helices: simple coiled-coils. Acta Crystallogr., 6(8-9):689-697, 1953.

${ }^{79}$ B. Y. Zhu, N. E. Zhou, C. M. Kay, and R. S. Hodges. Packing and hydrophobicity effects on protein folding and stability: effects of beta-branched amino acids, valine and isoleucine, on the formation and stability of two-stranded alpha-helical coiled coils/leucine zippers. Protein Sci., 2(3):383-94, 1993. 
${ }^{80}$ M. E. Massari and C. Murre. Helix-loop-helix proteins: regulators of transcription in eucaryotic organisms. Mol. Cell. Biol., 20(2):429-40, 2000

${ }^{81}$ P. S. McGrath, C. L. Watson, C. Ingram, M. A. Helmrath, and J. M. Wells. The basic helix-loophelix transcription factor neurog3 is required for development of the human endocrine pancreas. Diabetes, 64(7):2497-505, 2015.

${ }^{82}$ Sarah E. Ross, Michael E. Greenberg, and Charles D. Stiles. Basic helix-loop-helix factors in cortical development. Neuron, 39(1):13-25, 2003.

${ }^{83}$ R. Blake Hill, Daniel P. Raleigh, Angela Lombardi, and William F. Degrado. De novo design of helical bundles as models for understanding protein folding and function. Accounts Chem. Res., 33(11):745-754, 2000.

${ }^{84}$ Jennifer R. Litowski and Robert S. Hodges. Designing heterodimeric two-stranded $\alpha$-helical coiled-coils: Effects of hydrophobicity and $\alpha$-helical propensity on protein folding, stability, and specificity. F. Biol. Chem., 277(40):37272-37279, 2002.

${ }^{85}$ Franziska Thomas, Aimee L. Boyle, Antony J. Burton, and Derek N. Woolfson. A set of de novo designed parallel heterodimeric coiled coils with quantified dissociation constants in the micromolar to sub-nanomolar regime. J. Am. Chem. Soc., 135(13):5161-5166, 2013.

${ }^{86}$ P. B. Harbury, T. Zhang, P. S. Kim, and T. Alber. A switch between two-, three-, and four-stranded coiled coils in gcn4 leucine zipper mutants. Science, 262(5138):1401-7, 1993.

${ }^{87}$ Jason E. Donald, Daniel W. Kulp, and William F. DeGrado. Salt bridges: Geometrically specific, designable interactions. Proteins, 79(3):898-915, 2011.

${ }^{88}$ C. N. Pace and J. M. Scholtz. A helix propensity scale based on experimental studies of peptides and proteins. Biophys. F., 75(1):422-427, 1998.

${ }^{89}$ Erin K. O’Shea, Rheba Rutkowski, and Peter S. Kim. Mechanism of specificity in the fos-jun oncoprotein heterodimer. Cell, 68(4):699-708, 1992.

${ }^{90}$ S. Y. Lau, A. K. Taneja, and R. S. Hodges. Synthesis of a model protein of defined secondary and quaternary structure. effect of chain length on the stabilization and formation of two-stranded $\alpha$-helical coiled-coils. F. Biol. Chem., 259(21):13253-61, 1984.

${ }^{91}$ Johan Rydberg, Lars Baltzer, and Vijayalekshmi Sarojini. Intrinsically unstructured proteins by design-electrostatic interactions can control binding, folding, and function of a helix-loop-helix heterodimer. 7. Pept. Sci., 19(8):461-469, 2013.

92 Daniel Aili, Karin Enander, Johan Rydberg, Ingemar Lundström, Lars Baltzer, and Bo Liedberg. Aggregation-induced folding of a de novo designed polypeptide immobilized on gold nanoparticles. F. Am. Chem. Soc., 128(7):2194-2195, 2006.

${ }^{93}$ Daniel Aili, Karin Enander, Lars Baltzer, and Bo Liedberg. Assembly of polypeptide-functionalized gold nanoparticles through a heteroassociation- and folding-dependent bridging. Nano Lett., 8(8):2473-2478, 2008.

${ }^{94}$ Daniel Aili, Karin Enander, Johan Rydberg, Irina Nesterenko, Fredrik Björefors, Lars Baltzer, and Bo Liedberg. Folding induced assembly of polypeptide decorated gold nanoparticles. F. Am. Chem. Soc., 130(17):5780-5788, 2008.

95 T. Kimmerlin and D. Seebach. '100 years of peptide synthesis': ligation methods for peptide and protein synthesis with applications to beta-peptide assemblies. F. Pept. Res., 65(2):229-260, 2005.

96 "The Nobel Prize in Chemistry 1984". Nobelprize.org. Web 29 Oct 2016.

${ }^{97}$ M. Amblard, J. A. Fehrentz, J. Martinez, and G. Subra. Methods and protocols of modern solid phase peptide synthesis. Mol. Biotechnol., 33(3):239-54, 2006.

${ }^{98}$ C. D. Chang and J. Meienhofer. Solid-phase peptide synthesis using mild base cleavage of $\mathrm{n}$ alphafluorenylmethyloxycarbonylamino acids, exemplified by a synthesis of dihydrosomatostatin. Int.F. Pept. Protein Res., 11(3):246-9, 1978.

${ }^{99}$ Jöns Jakob Berzelius. Årsberättelse om framstegen i fysik och kemi, afgiven den 31 mars 1832. Norstedt, Stockholm, 1832. 
${ }^{100}$ Commission on Macromolecular Nomenclature. Glossary of basic terms in polymer science. Pure \& Appl. Chem., 68(12):2287-2311, 1996.

${ }^{101}$ A. Fakhari and C. Berkland. Applications and emerging trends of hyaluronic acid in tissue engineering, as a dermal filler and in osteoarthritis treatment. Acta Biomaterial., 9(7):7081-7092, 2013.

${ }^{102}$ Karl Meyer and John W. Palmer. The polysaccharide of the vitreous humor. F. Biol.l Chem., 107(3):629-634, 1934.

${ }^{103}$ Suniti Misra, Vincent C. Hascall, Roger R. Markwald, and Shibnath Ghatak. Interactions between hyaluronan and its receptors (cd44, rhamm) regulate the activities of inflammation and cancer. Front. Immunol., 6:201, 2015.

104 J. Necas, L. Bartosikova, P. Brauner, and J. Kolar. Hyaluronic acid (hyaluronan): a review. Vet. Med. Czech., 53(8):397-411, 2008.

${ }^{105}$ Kevin T. Dicker, Lisa A. Gurski, Swati Pradhan-Bhatt, Robert L. Witt, Mary C. Farach-Carson, and Xinqiao Jia. Hyaluronan: A simple polysaccharide with diverse biological functions. Acta Biomater., 10(4):1558-1570, 2014.

${ }^{106}$ Akira Takahashi, Yukimitsu Suzuki, Takashi Suhara, Kiyohiko Omichi, Atsushi Shimizu, Kiyoshi Hasegawa, Norihiro Kokudo, Seiichi Ohta, and Taichi Ito. In situ cross-linkable hydrogel of hyaluronan produced via copper-free click chemistry. Biomacromolecules, 14(10):3581-3588, 2013.

${ }^{107}$ Sudhir Khetan, Murat Guvendiren, Wesley R. Legant, Daniel M. Cohen, Christopher S. Chen, and Jason A. Burdick. Degradation-mediated cellular traction directs stem cell fate in covalently crosslinked three-dimensional hydrogels. Nat. Mater., 12(5):458-465, 2013.

${ }^{108}$ Xin Zhang, Pengcheng Sun, Lingzi Huangshan, Bi-Huang Hu, and Phillip B. Messersmith. Improved method for synthesis of cysteine modified hyaluronic acid for in situ hydrogel formation. Chem. Commun., 51(47):9662-9665, 2015.

${ }^{109}$ Fabrizio Picotti, Matteo Fabbian, Rita Gianni, Alessandra Sechi, Luca Stucchi, and Marco Bosco. Hyaluronic acid lipoate: Synthesis and physicochemical properties. Carbohydr. Polym., 93(1):273$278,2013$.

${ }^{110}$ Christopher B. Rodell, Adam L. Kaminski, and Jason A. Burdick. Rational design of network properties in guest-host assembled and shear-thinning hyaluronic acid hydrogels. Biomacromolecules, 14(11):4125-4134, 2013.

${ }^{111}$ Stefania Federico, Ulrich Nöchel, Candy Löwenberg, Andreas Lendlein, and Axel T. Neffe. Supramolecular hydrogel networks formed by molecular recognition of collagen and a peptide grafted to hyaluronic acid. Acta Biomaterial., 38:1-10, 2016.

${ }^{112}$ Xubo Zhao, Xu Jia, Lei Liu, Jin Zeng, Kun Tian, Tingting Zhou, and Peng Liu. Doublecross-linked hyaluronic acid nanoparticles with $\mathrm{ph} /$ reduction dual-responsive triggered release and ph-modulated fluorescence for folate-receptor-mediated targeting visualized chemotherapy. Biomacromolecules, 17(4):1496-1505, 2016.

${ }^{113}$ Ki Young Choi, Hyunjin Chung, Kyung Hyun Min, Hong Yeol Yoon, Kwangmeyung Kim, Jae Hyung Park, Ick Chan Kwon, and Seo Young Jeong. Self-assembled hyaluronic acid nanoparticles for active tumor targeting. Biomaterials, 31(1):106-114, 2010.

${ }^{114}$ Jisoo Shin, Jung Seung Lee, Changhyun Lee, Hyun-Ji Park, Kisuk Yang, Yoonhee Jin, Ji Hyun Ryu, Ki Sung Hong, Sung-Hwan Moon, Hyung-Min Chung, Hee Seok Yang, Soong Ho Um, Jong-Won Oh, Dong-Ik Kim, Haeshin Lee, and Seung-Woo Cho. Tissue adhesive catecholmodified hyaluronic acid hydrogel for effective, minimally invasive cell therapy. Adv. Funct. Mater., 25(25):3814-3824, 2015.

${ }^{115}$ Katrin Knop, Richard Hoogenboom, Dagmar Fischer, and Ulrich S Schubert. Poly(ethylene glycol) in drug delivery: Pros and cons as well as potential alternatives. Angew. Chem. Int. Edit., 49(36):6288-6308, 2010.

${ }^{116}$ F. M. Veronese and A. Mero. The impact of pegylation on biological therapies. BioDrugs, 22(5):31529, 2008. 
${ }^{117}$ A. Worz, B. Berchtold, K. Moosmann, O. Prucker, and J. Ruhe. Protein-resistant polymer surfaces. f. Mater. Chem., 22(37):19547-19561, 2012.

${ }^{118}$ Seraphine V. Wegner, Franziska C. Schenk, Sina Witzel, Friedrich Bialas, and Joachim P. Spatz. Cobalt cross-linked redox-responsive peg hydrogels: From viscoelastic liquids to elastic solids. Macromolecules, 49(11):4229-4235, 2016.

${ }^{119}$ Jianfeng Zhang, Ben Muirhead, Megan Dodd, Lina Liu, Fei Xu, Nicole Mangiacotte, Todd Hoare, and Heather Sheardown. An injectable hydrogel prepared using a peg/vitamin e copolymer facilitating aqueous-driven gelation. Biomacromolecules, 2016.

${ }^{120}$ Dianyu Dong, Junjie Li, Man Cui, Jinmei Wang, Yuhang Zhou, Liu Luo, Yufei Wei, Lei Ye, Hong Sun, and Fanglian Yao. In situ "clickable" zwitterionic starch-based hydrogel for 3d cell encapsulation. ACS Appl. Mater. Interfaces, 8(7):4442-4455, 2016.

${ }^{121}$ Zhao Li, Zhen Zheng, Shan Su, Lin Yu, and Xinling Wang. Preparation of a high-strength hydrogel with slidable and tunable potential functionalization sites. Macromolecules, 49(1):373-386, 2016.

${ }^{122}$ Malar A. Azagarsamy, Ian A. Marozas, Sergio Spaans, and Kristi S. Anseth. Photoregulated hydrazone-based hydrogel formation for biochemically patterning $3 \mathrm{~d}$ cellular microenvironments. ACS Macro Lett., 5(1):19-23, 2016.

${ }^{123}$ R. Ravichandran, M. M. Islam, E. I. Alarcon, A. Samanta, S. Wang, P. Lundstrom, J. Hilborn, M. Griffith, and J. Phopase. Functionalised type-i collagen as a hydrogel building block for bioorthogonal tissue engineering applications. F. Mater. Chem. B, 4(2):318-326, 2016.

${ }^{124}$ Christopher M. Madl, Lily M. Katz, and Sarah C. Heilshorn. Bio-orthogonally crosslinked, engineered protein hydrogels with tunable mechanics and biochemistry for cell encapsulation. Adv. Funct. Mater., 26(21):3612-3620, 2016.

${ }^{125}$ Erin Smith, Jennifer Yang, Locksley McGann, Walter Sebald, and Hasan Uludag. Rgd-grafted thermoreversible polymers to facilitate attachment of bmp- 2 responsive c $2 \mathrm{c} 12$ cells. Biomaterials, 26(35):7329-7338, 2005.

${ }^{126}$ Junmin Zhu. Bioactive modification of poly(ethylene glycol) hydrogels for tissue engineering. Biomaterials, 31(17):4639-4656, 2010.

${ }^{127}$ Christopher D. Hein, Xin-Ming Liu, and Dong Wang. Click chemistry, a powerful tool for pharmaceutical sciences. Pharmaceut. Res., 25(10):2216-2230, 2008.

${ }^{128}$ Brian H. Northrop, Stephen H. Frayne, and Umesh Choudhary. Thiol-maleimide "click" chemistry: evaluating the influence of solvent, initiator, and thiol on the reaction mechanism, kinetics, and selectivity. Polym. Chem., 6(18):3415-3430, 2015.

${ }^{129}$ Oleksandr Koniev and Alain Wagner. Developments and recent advancements in the field of endogenous amino acid selective bond forming reactions for bioconjugation. Chem. Soc. Rev., 44(15):5495-5551, 2015.

${ }^{130}$ H. C. Kolb, M. G. Finn, and K. B. Sharpless. Click chemistry: Diverse chemical function from a few good reactions. Angew. Chem. Int. Ed., 40(11):2004-2021, 2001.

${ }^{131}$ Ngalle Eric Mbua, Jun Guo, Margreet A. Wolfert, Richard Steet, and Geert-Jan Boons. Strainpromoted alkyne-azide cycloadditions (spaac) reveal new features of glycoconjugate biosynthesis. Chembiochem, 12(12):1912-1921, 2011.

${ }^{132}$ Jan Dommerholt, Olivia van Rooijen, Annika Borrmann, Célia Fonseca Guerra, F. Matthias Bickelhaupt, and Floris L. van Delft. Highly accelerated inverse electron-demand cycloaddition of electron-deficient azides with aliphatic cyclooctynes. Nat. Commun., 5:5378, 2014.

${ }^{133}$ Marjoke F. Debets, Sander S. van Berkel, Jan Dommerholt, A. J. Dirks, Floris P. J. T. Rutjes, and Floris L. van Delft. Bioconjugation with strained alkenes and alkynes. Accounts Chem. Res., 44(9):805-815, 2011.

${ }^{134}$ F. Thomas, N. C. Burgess, A. R. Thomson, and D. N. Woolfson. Controlling the assembly of coiledcoil peptide nanotubes. Angew. Chem. Int. Ed., 55(3):987-91, 2016.

${ }^{135}$ Chengyi Song, Yang Wang, and Nathaniel L. Rosi. Peptide-directed synthesis and assembly of hollow spherical copt nanoparticle superstructures. Angew. Chem. Int. Edit., 52(14):3993-3995, 2013. 
${ }^{136}$ Marcos R. Guilherme, Fauze A. Aouada, André R. Fajardo, Alessandro F. Martins, Alexandre T. Paulino, Magali F. T. Davi, Adley F. Rubira, and Edvani C. Muniz. Superabsorbent hydrogels based on polysaccharides for application in agriculture as soil conditioner and nutrient carrier: A review. Eur. Polym. 7., 72:365-385, 2015.

${ }^{137}$ Paul C. Nicolson and Jürgen Vogt. Soft contact lens polymers: an evolution. Biomaterials, 22(24):3273-3283, 2001.

138 Todd R. Hoare and Daniel S. Kohane. Hydrogels in drug delivery: Progress and challenges. Polymer, 49(8):1993-2007, 2008.

${ }^{139}$ Daniel Buenger, Fuat Topuz, and Juergen Groll. Hydrogels in sensing applications. Prog. Polym. Sci., 37(12):1678-1719, 2012.

${ }^{140}$ John A. Hunt, Rui Chen, Theun van Veen, and Nicholas Bryan. Hydrogels for tissue engineering and regenerative medicine. F. Mater. Chem. B, 2(33):5319-5338, 2014.

${ }^{141}$ Marta Madaghiele, Christian Demitri, Alessandro Sannino, and Luigi Ambrosio. Polymeric hydrogels for burn wound care: Advanced skin wound dressings and regenerative templates. Burns Trauma, 2(4):153-161, 2014.

${ }^{142}$ M. E. Parente, A. Ochoa Andrade, G. Ares, F. Russo, and A. Jimenez-Kairuz. Bioadhesive hydrogels for cosmetic applications. Int. F. Cosmet. Sci., 37(5):511-8, 2015.

${ }^{143}$ Mark W. Tibbitt and Kristi S. Anseth. Hydrogels as extracellular matrix mimics for $3 \mathrm{~d}$ cell culture. Biotechnol. Bioeng., 103(4):655-663, 2009.

${ }^{144}$ Steven R. Caliari and Jason A. Burdick. A practical guide to hydrogels for cell culture. Nat. Meth., 13(5):405-414, 2016.

${ }^{145}$ Peter Worthington, Darrin J. Pochan, and Sigrid A. Langhans. Peptide hydrogels - versatile matrices for 3d cell culture in cancer medicine. Front. Oncol., 5(92), 2015.

${ }^{146}$ Adam J. Engler, Shamik Sen, H. Lee Sweeney, and Dennis E. Discher. Matrix elasticity directs stem cell lineage specification. Cell, 126(4):677-689, 2006.

${ }^{147}$ Ruijiao Dong, Yan Pang, Yue Su, and Xinyuan Zhu. Supramolecular hydrogels: synthesis, properties and their biomedical applications. Biomat. Sci., 3(7):937-954, 2015.

${ }^{148}$ F. Schaffner. Hepatic drug metabolism and adverse hepatic drug reactions. Vet. Pathol., 12(2):14556, 1975.

${ }^{149}$ F. Berthiaume, P. V. Moghe, M. Toner, and M. L. Yarmush. Effect of extracellular matrix topology on cell structure, function, and physiological responsiveness: hepatocytes cultured in a sandwich configuration. Faseb. F., 10(13):1471-84, 1996.

${ }^{150}$ E. L. LeCluyse, K. L. Audus, and J. H. Hochman. Formation of extensive canalicular networks by rat hepatocytes cultured in collagen-sandwich configuration. Am. f. Physiol., 266(6 Pt 1):C1764-74, 1994.

${ }^{151}$ Patricio Godoy, Nicola J. Hewitt, Ute Albrecht, Melvin E. Andersen, Nariman Ansari, Sudin Bhattacharya, Johannes Georg Bode, Jennifer Bolleyn, Christoph Borner, Jan Böttger, Albert Braeuning, Robert A. Budinsky, Britta Burkhardt, Neil R. Cameron, Giovanni Camussi, ChongSu Cho, Yun-Jaie Choi, J. Craig Rowlands, Uta Dahmen, Georg Damm, Olaf Dirsch, María Teresa Donato, Jian Dong, Steven Dooley, Dirk Drasdo, Rowena Eakins, Karine Sá Ferreira, Valentina Fonsato, Joanna Fraczek, Rolf Gebhardt, Andrew Gibson, Matthias Glanemann, Chris E. P. Goldring, María José Gómez-Lechón, Geny M. M. Groothuis, Lena Gustavsson, Christelle Guyot, David Hallifax, Seddik Hammad, Adam Hayward, Dieter Häussinger, Claus Hellerbrand, Philip Hewitt, Stefan Hoehme, Hermann-Georg Holzhütter, J. Brian Houston, Jens Hrach, Kiyomi Ito, Hartmut Jaeschke, Verena Keitel, Jens M. Kelm, B. Kevin Park, Claus Kordes, Gerd A. KullakUblick, Edward L. LeCluyse, Peng Lu, Jennifer Luebke-Wheeler, Anna Lutz, Daniel J. Maltman, Madlen Matz-Soja, Patrick McMullen, Irmgard Merfort, Simon Messner, Christoph Meyer, Jessica Mwinyi, Dean J. Naisbitt, Andreas K. Nussler, Peter Olinga, Francesco Pampaloni, Jingbo Pi, Linda Pluta, Stefan A. Przyborski, Anup Ramachandran, Vera Rogiers, Cliff Rowe, Celine Schelcher, Kathrin Schmich, Michael Schwarz, Bijay Singh, Ernst H. K. Stelzer, Bruno Stieger, Regina Stöber, Yuichi Sugiyama, Ciro Tetta, Wolfgang E. Thasler, Tamara Vanhaecke, Mathieu Vinken, Thomas S. 
Weiss, Agata Widera, Courtney G. Woods, Jinghai James Xu, Kathy M. Yarborough, and Jan G. Hengstler. Recent advances in $2 \mathrm{~d}$ and $3 \mathrm{~d}$ in vitro systems using primary hepatocytes, alternative hepatocyte sources and non-parenchymal liver cells and their use in investigating mechanisms of hepatotoxicity, cell signaling and adme. Arch. Toxicol., 87(8):1315-1530, 2013.

${ }^{152}$ R. Carlsson, E. Engvall, A. Freeman, and E. Ruoslahti. Laminin and fibronectin in cell adhesion: enhanced adhesion of cells from regenerating liver to laminin. Proc. Natl. Acad. Sci. USA, 78(4):2403-6, 1981.

${ }^{153}$ B. Clement, B. Segui-Real, P. Savagner, H. K. Kleinman, and Y. Yamada. Hepatocyte attachment to laminin is mediated through multiple receptors. F. Cell Biol., 110(1):185-92, 1990.

${ }^{154}$ Sreenivasa C. Ramaiahgari, Michiel W. den Braver, Bram Herpers, Valeska Terpstra, Jan N. M. Commandeur, Bob van de Water, and Leo S. Price. A 3d in vitro model of differentiated hepg2 cell spheroids with improved liver-like properties for repeated dose high-throughput toxicity studies. Arch. Toxicol., 88(5):1083-1095, 2014.

${ }^{155}$ Claudia Luckert, Christina Schulz, Nadja Lehmann, Maria Thomas, Ute Hofmann, Seddik Hammad, Jan G. Hengstler, Albert Braeuning, Alfonso Lampen, and Stefanie Hessel. Comparative analysis of 3d culture methods on human hepg2 cells. Arch. Toxicol., pages 1-14, 2016.

${ }^{156}$ Amish Asthana and William S. Kisaalita. Microtissue size and hypoxia in hts with $3 \mathrm{~d}$ cultures. Drug Discov. Today, 17(15-16):810-817, 2012.

${ }^{157}$ N. J. Darling, Y. S. Hung, S. Sharma, and T. Segura. Controlling the kinetics of thiol-maleimide michael-type addition gelation kinetics for the generation of homogenous poly(ethylene glycol) hydrogels. Biomaterials, 101:199-206, 2016.

${ }^{158}$ Prathamesh M. Kharkar, Matthew S. Rehmann, Kelsi M. Skeens, Emanual Maverakis, and April M. Kloxin. Thiol-ene click hydrogels for therapeutic delivery. ACS Biomater. Sci. Eng., 2(2):165-179, 2016 .

${ }^{159}$ B. Trzaskowski, L. Adamowicz, and P. A. Deymier. A theoretical study of zinc(ii) interactions with amino acid models and peptide fragments. F. Biol. Inorg. Chem., 13(1):133-7, 2008.

${ }^{160}$ T. A. Witten and L. M. Sander. Diffusion-limited aggregation, a kinetic critical phenomenon. Phys. Rev. Lett., 47(19):1400-1403, 1981.

${ }^{161}$ Andreas Åslund, Christina J. Sigurdson, Therése Klingstedt, Stefan Grathwohl, Tristan Bolmont, Dara L. Dickstein, Eirik Glimsdal, Stefan Prokop, Mikael Lindgren, Peter Konradsson, David M. Holtzman, Patrick R. Hof, Frank L. Heppner, Samuel Gandy, Mathias Jucker, Adriano Aguzzi, Per Hammarström, and K. Peter R. Nilsson. Novel pentameric thiophene derivatives for in vitro and in vivo optical imaging of a plethora of protein aggregates in cerebral amyloidoses. ACS Chem. Biol., 4(8):673-684, 2009.

162 Chaoyang Jiang, Sergiy Markutsya, Yuri Pikus, and Vladimir V. Tsukruk. Freely suspended nanocomposite membranes as highly sensitive sensors. Nat. Mater., 3(10):721-728, 2004.

${ }^{163}$ C. Jenny $\mathrm{Mu}$, David A. LaVan, Robert S. Langer, and Bruce R. Zetter. Self-assembled gold nanoparticle molecular probes for detecting proteolytic activity in vivo. ACS Nano, 4(3):1511$1520,2010$.

${ }^{164}$ L. Civitelli, L. Sandin, E. Nelson, S. I. Khattak, A. C. Brorsson, and K. Kagedal. The luminescent oligothiophene p-ftaa converts toxic abeta1-42 species into nontoxic amyloid fibers with altered properties. F. Biol. Chem., 291(17):9233-43, 2016.

${ }^{165}$ U. S. Herrmann, A. K. Schutz, H. Shirani, D. Huang, D. Saban, M. Nuvolone, B. Li, B. Ballmer, A. K. Aslund, J. J. Mason, E. Rushing, H. Budka, S. Nystrom, P. Hammarstrom, A. Bockmann, A. Caflisch, B. H. Meier, K. P. Nilsson, S. Hornemann, and A. Aguzzi. Structure-based drug design identifies polythiophenes as antiprion compounds. Sci. Transl. Med., 7(299):299ra123, 2015.

${ }^{166}$ I. Margalith, C. Suter, B. Ballmer, P. Schwarz, C. Tiberi, T. Sonati, J. Falsig, S. Nystrom, P. Hammarstrom, A. Aslund, K. P. Nilsson, A. Yam, E. Whitters, S. Hornemann, and A. Aguzzi. Polythiophenes inhibit prion propagation by stabilizing prion protein (prp) aggregates. $\mathrm{f}$. Biol. Chem., 287(23):18872-87, 2012. 
${ }^{167}$ Erik Martinsson, Borja Sepulveda, Peng Chen, Anders Elfwing, Bo Liedberg, and Daniel Aili. Optimizing the refractive index sensitivity of plasmonically coupled gold nanoparticles. Plasmonics, 9(4):773-780, 2014.

${ }^{168}$ Nazanin Roohani, Richard Hurrell, Roya Kelishadi, and Rainer Schulin. Zinc and its importance for human health: An integrative review. F. Res. Med. Sci., 18(2):144-157, 2013.

${ }^{169}$ Rebecca A. Bozym, Fabrice Chimienti, Leonard J. Giblin, Gunter W. Gross, Irina Korichneva, Yuan Li, Sarah Libert, Wolfgang Maret, Maryam Parviz, Christopher J. Frederickson, and Richard B. Thompson. Free zinc ions outside a narrow concentration range are toxic to a variety of cells in vitro. Exp. Biol. Med., 235(6):741-750, 2010.

${ }^{170}$ Mahmoud S. Hassan and Haytham M. M. Ibrahim. Characterization and antimicrobial properties of metal complexes of polypropylene fibers grafted with acrylic acid using gamma irradiation. Polym. Adv. Tech., 27(4):532-541, 2016.

${ }^{171}$ Chao Xu, Yanbin Cai, Chunhua Ren, Jie Gao, and Jihui Hao. Zinc-triggered hydrogelation of selfassembled small molecules to inhibit bacterial growth. Sci. Rep., 5:7753, 2015.

${ }^{172}$ A. B. Lansdown, U. Mirastschijski, N. Stubbs, E. Scanlon, and M. S. Agren. Zinc in wound healing: theoretical, experimental, and clinical aspects. Wound Repair Regen., 15(1):2-16, 2007.

${ }^{173}$ Monali Mahedia, Nilay Shah, and Bardia Amirlak. Clinical evaluation of hyaluronic acid sponge with zinc versus placebo for scar reduction after breast surgery. Plast. Reconstr. Surg. Glob. Open, 4(7):e791, 2016.

${ }^{174}$ I. Juhász, P. Zoltán, and I. Erdei. Treatment of partial thickness burns with zn-hyaluronan: lessons of a clinical pilot study. Ann. Burns Fire Disasters, 25(2):82-85, 2012.

${ }^{175} \mathrm{~J}$ A Tamada and R Langer. Erosion kinetics of hydrolytically degradable polymers. Proc. Natl. Acad. Sci. USA, 90(2):552-556, 1993.

${ }^{176}$ L. Šoltés, R. Mendichi, G. Kogan, J. Schiller, M. Stankovská, and J. Arnhold. Degradative action of reactive oxygen species on hyaluronan. Biomacromolecules, 7(3):659-668, 2006.

${ }^{177}$ György T. Balogh, János Illés, Zsuzsanna Székely, Erika Forrai, and Anikó Gere. Effect of different metal ions on the oxidative damage and antioxidant capacity of hyaluronic acid. Arch. Biochem. Biophys., 410(1):76-82, 2003.

${ }^{178}$ Chunlin Ke, Lanping Sun, Deliang Qiao, Di Wang, and Xiaoxiong Zeng. Antioxidant acitivity of low molecular weight hyaluronic acid. Food Chem. Toxicol., 49(10):2670-2675, 2011.

${ }^{179}$ T. C. Barnes, M. E. Anderson, S. W. Edwards, and R. J. Moots. Neutrophil-derived reactive oxygen species in ssc. Rheumatology, 51(7):1166-9, 2012.

${ }^{180}$ J. Weickenmeier, R. de Rooij, S. Budday, P. Steinmann, T. C. Ovaert, and E. Kuhl. Brain stiffness increases with myelin content. Acta Biomater., 42:265-272, 2016.

${ }^{181}$ D. Campbell, R. A. Pethrick, and J. R. White. Polymer characterization : physical techniques. S. Thornes, Cheltenham, Glos., U.K., 2000.

${ }^{182}$ S. M. Kelly and N. C. Price. The use of circular dichroism in the investigation of protein structure and function. Curr. Protein. Pept. Sci., 1(4):349-84, 2000.

${ }^{183}$ Norma J. Greenfield and Gerald D. Fasman. Computed circular dichroism spectra for the evaluation of protein conformation. Biochemistry, 8(10):4108-4116, 1969.

${ }^{184}$ Marilyn Emerson Holtzer and Alfred Holtzer. $\alpha$-helix to random coil transitions: Determination of peptide concentration from the cd at the isodichroic point. Biopolymers, 32(12):1675-1677, 1992.

${ }^{185}$ W. I. Goldburg. Dynamic light scattering. Am. F. Phys., 67(12):1152-1160, 1999.

${ }^{186}$ Joseph R. Lakowicz. Principles of fluorescence spectroscopy. Springer, New York, 2006.

${ }^{187}$ Michael W. Davidson. Ernst abbe. Lab. Med., 40(8):502-503, 2009.

188 "The Nobel Prize in Chemistry 2014". Nobelprize.org. Web 11 Nov 2016.

${ }^{189}$ Judith C. Yang, Matthew W. Small, Ross V. Grieshaber, and Ralph G. Nuzzo. Recent developments and applications of electron microscopy to heterogeneous catalysis. Chem. Soc. Rev., 41(24):81798194, 2012. 
${ }^{190}$ Indra J. Das, Chee-Wai Cheng, Raj K. Mitra, Alireza Kassaee, Zelig Tochner, and Lawrence J. Solin. Transmission and dose perturbations with high-z materials in clinical electron beams. Med. Phys., 31(12):3213-3221, 2004.

${ }^{191}$ Christian Hahnel, Susanne Somodi, Christine Slowik, Dieter G. Weiss, and Rudolf F. Guthoff. Fluorescence microscopy and three-dimensional imaging of the porcine corneal keratocyte network. Graefes Arch. Clin. Exp. Ophthalmol., 235(12):773-779, 1997.

192 Alexander Ya Malkin and Avraam I. Isayev. Rheology : Concepts, Methods, and Applications. ChemTech Publishing, Toronto, 2012. 



\section{Publications}

The articles associated with this thesis have been removed for copyright reasons. For more details about these see:

http://urn.kb.se/resolve?urn=urn:nbn:se:liu:diva-132949 